\title{
Small decision, big impact : promoting influenza vaccination uptake among health care workers
}

Citation for published version (APA):

Lehmann, B. A. (2015). Small decision, big impact : promoting influenza vaccination uptake among health care workers. [Doctoral Thesis, Maastricht University]. Datawyse / Universitaire Pers Maastricht. https://doi.org/10.26481/dis.20151016bl

Document status and date:

Published: 01/01/2015

DOI:

10.26481/dis.20151016bl

Document Version:

Publisher's PDF, also known as Version of record

\section{Please check the document version of this publication:}

- A submitted manuscript is the version of the article upon submission and before peer-review. There can be important differences between the submitted version and the official published version of record.

People interested in the research are advised to contact the author for the final version of the publication, or visit the DOI to the publisher's website.

- The final author version and the galley proof are versions of the publication after peer review.

- The final published version features the final layout of the paper including the volume, issue and page numbers.

Link to publication

\footnotetext{
General rights rights.

- You may freely distribute the URL identifying the publication in the public portal. please follow below link for the End User Agreement:

www.umlib.nl/taverne-license

Take down policy

If you believe that this document breaches copyright please contact us at:

repository@maastrichtuniversity.nl

providing details and we will investigate your claim.
}

Copyright and moral rights for the publications made accessible in the public portal are retained by the authors and/or other copyright owners and it is a condition of accessing publications that users recognise and abide by the legal requirements associated with these

- Users may download and print one copy of any publication from the public portal for the purpose of private study or research.

- You may not further distribute the material or use it for any profit-making activity or commercial gain

If the publication is distributed under the terms of Article $25 \mathrm{fa}$ of the Dutch Copyright Act, indicated by the "Taverne" license above, 


\section{Small decision, big impact}

Promoting influenza vaccination uptake among health care workers

Birthe A. Lehmann 
(C) Copyright Birthe Andrea Lehmann, Maastricht 2015 Production: Datawyse | Universitaire Pers Maastricht Cover design: Frank Peeters, DOC

ISBN: 9789461594617 


\title{
Small decision, big impact
}

\author{
Promoting influenza vaccination uptake \\ among health care workers
}

\begin{abstract}
DISSERTATION
to obtain the degree of Doctor at Maastricht University, on the authority of the Rector Magnificus, Prof. Dr. L.L.G. Soete in accordance with the decision of the Board of Deans, to be defended in public on Friday, October 16, 2015 at 12.00 hours
\end{abstract}

by

Birthe Andrea Lehmann

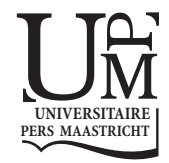




\section{Supervisors}

Prof. Dr. R.A.C. Ruiter

Prof. Dr. G. Kok

Prof. Dr. G.B. Chapman (Rutgers University)

\section{Assessment Committee}

Prof. Dr. F.R.H. Zijlstra (chairman)

Prof. Dr. T. van Achterberg (KU Leuven)

Prof. Dr. C.J.P.A. Hoebe

Prof. Dr. K. Horstman

Dr. T.G.W.M. Paulussen (TNO Leiden) 


\section{Table of contents}

$\begin{array}{lll}\text { Chapter } 1 & \text { Introduction } & 7\end{array}$

Chapter 2 A qualitative study of the coverage of influenza vaccination on

Dutch news sites and social media websites

Chapter 3 Medical students' attitude towards influenza vaccination

Chapter $4 \quad$ "I don't see an added value for myself"

Chapter 5 Social-cognitive predictors of health care workers' intention to get vaccinated against influenza in Belgian, Dutch and German hospital settings

Chapter 6 The intention to get vaccinated against influenza and actual vaccination uptake of Dutch health care workers

Chapter 7 Changing the default to promote influenza vaccination among health care workers

Chapter 8 General Discussion

References

Valorization

Summary

Samenvatting

Zusammenfassung

Acknowledgements

Curriculum vitae 

Introduction 



\section{INTRODUCTION}

Influenza epidemics are a major public health problem causing severe morbidity and mortality every year. Annual epidemics affect 5 to $10 \%$ of adults and 20 to $30 \%$ of children worldwide. They result in up to five million cases of severe illness and death (WHO, 2014). In Europe, the estimated deaths accountable to annual influenza epidemics range between 40.000 and 220.000 , depending on the severity of the epidemic (Commission of the European Communities, 2009). Affected are especially young children, the elderly, and people with underlying chronic diseases (Mertz et al., 2013; WHO, 2014). They can get infected by relatives, other patients they come in contact with using health care services, and health care workers (HCWs). Health Authorities recommend annual influenza vaccination of HCWs, because it has shown to decrease the transmission of influenza to vulnerable patients and to prevent influenza-related work-absenteeism in this group (Gezondheidsraad, 2014; WHO, 2014). Costs resulting from influenza-related medical treatments and work-absenteeism present a considerable socioeconomic burden (Commission of the European Communities, 2009).

Despite the evidence for the effectiveness of vaccination in protecting patients and decreasing work-absenteeism among HCWs, vaccination coverage rates are low in Europe (Blank, Schwenkglenks, \& Szucs, 2009; Endrich, Blank, \& Szucs, 2009). The objective of this dissertation is to gain more insight into the reasons why coverage rates are low among HCWs and to apply Intervention Mapping (IM), a planning process for the systematic theory- and evidence-based development of health promotion interventions, to the development of an intervention to promote influenza vaccination uptake among HCWs.

This chapter covers information on seasonal influenza and influenza vaccination, which is followed by an overview of the reasons why HCWs should get vaccinated against influenza and existing knowledge about their reasons for (not) getting vaccinated. The influence of media coverage about vaccinations on the mistrust towards vaccinations will be described. Finally, we give an overview of previous attempts undertaken to increase influenza vaccination uptake among HCWs.

\section{Seasonal influenza}

Influenza is an infectious disease of the respiratory tract that is currently caused by one of two influenza subtype A viruses (H1N1/ H3N2) and an influenza B virus (WHO, 2012). Influenza is a vaccine preventable disease, however influenza viruses change constantly, which is why people do not develop lifelong immunity after being vaccinated, as it can be the case for other viruses. For this reason, the composition of the vaccine has to be adjusted every year. Influenza is very contagious and everyone can get infected independent of age or health status. It mainly spreads through coughing and sneezing; little droplets enter susceptible cells of the respiratory track through the air. 
But the virus also spreads indirectly, through contact with contagious surfaces, such as door handles or telephones (WHO, 2012).

Most people that contract the virus suffer from a mild form of influenza that can still lead to considerable work-absenteeism. However, some people have an increased risk of suffering from severe complications following an infection with influenza. The most common, in some cases life-threatening complications following an influenza infection are pneumonia, ear infection, myocarditis, and other bacterial infections (WHO, 2014). Belonging to the risk groups are children younger than 2 years of age, people aged 65 years or older, pregnant women, and people of any age with certain medical conditions, such as chronic heart, lung, kidney, liver, blood or metabolic diseases (i.e. diabetes), or a weakened immune system (Gezondheidsraad, 2014; WHO, 2014). The Dutch Health Council advises the vaccination of people from age 60 onwards and does not support the WHO recommendation to vaccinate pregnant women (Gezondheidsraad, 2014).

\section{Influenza vaccination}

The most effective way to prevent infection with an influenza virus, and possible severe complications from it, is vaccination. Influenza vaccines are available for more than 60 years and have been shown to be safe and reasonably effective (WHO, 2014). The influenza vaccine contains inactivated pieces of usually three to four virus subtypes that are expected in the upcoming winter season (WHO, 2012). Influenza vaccination is most effective when the virus subtypes included in the vaccine and the circulating virus subtypes match well. For this purpose, the WHO Global Influenza Surveillance and Response System (GISRS) monitors the influenza viruses circulating in humans and formulate recommendations (WHO, 2014).

The immune system reacts on the vaccine by developing antibodies against the influenza virus, which usually proceeds with minimal side-effects, if any. Protection lasts for about four to six months. If a person gets influenza despite being vaccinated, then the illness will likely proceed in a less severe form (WHO, 2014). For people with a weakened immune system, such as the elderly, effectiveness of the vaccine has been shown to be reduced, but may still decrease hospitalization by $25-39 \%$ and has been shown to reduce overall mortality by 39-75\% (Beyer et al., 2013; WHO, 2005). For healthy adults, effectiveness of the vaccines has been shown to be good (WHO, 2012). Laboratory-confirmed illness was shown to be reduced by 70-90\% (Commission of the European Communities, 2009).

\section{Influenza vaccination for health care workers}

People belonging to the high risk groups are the ones that are also the most likely to regularly make use of health care services. As patients, they can contract influenza from other patients, visitors and from health care workers (HCWs). Influenza vaccina- 
tion of HCWs can decrease the transmission of influenza to vulnerable patients. Previous research has shown that vaccination of HCWs can decrease clinical disease in healthy adults by $70-90 \%$, it can reduce all-cause mortality in long-term care patients, such as elderly home residents by up to $29 \%$ (Ahmed, Lindley, Allred, Weinbaum, \& Grohskopf, 2014; WHO, 2005), and might have a similar or an even higher impact on patients in hospitals (Amodio et al., 2014; Bénet et al., 2012; Macesic, Kotsimbos, Kelly, \& Cheng, 2013; Van den Dool, Bonten, Hak, \& Wallinga, 2009).

National and international Health Authorities, such as the Dutch Health Council and the World Health Organization, therefore recommend annual influenza vaccination for HCWs (Gezondheidsraad, 2011; WHO, 2014). The reasons why HCWs should get vaccinated against influenza annually are threefold: (1) as mentioned above, vaccinating HCWs can prevent hospital-acquired or nosocomial infections, (2) vaccination will also protect the HCW, his/her family members and colleagues, and (3) it will likely reduce the economic burden of influenza by decreasing influenza-related workabsenteeism.

First of all, an increasing amount of evidence points to the conclusion that vaccinating nursing staff and physicians helps prevent influenza infection among patients; the more HCWs are vaccinated against influenza, the fewer patients acquire influenza and/ or influenza-related (life threatening) infections, such as pneumonia within a hospital (Amodio et al., 2014). The prevention of hospital-acquired or nosocomial influenza is an important objective for infection control and patient safety within hospitals. Several studies report that influenza infection can lead to nosocomial outbreaks with negative consequences for patients, as well as the health care organization (Amodio et al., 2014; Bénet et al., 2012; Salgado, Farr, Hall, \& Hayden, 2002; Sartor et al., 2002; Voirin, Barret, Metzger, \& Vanhems, 2009). Bénet and colleagues (2012) found in their study that $20 \%$ of patients contracted influenza during a nosocomial outbreak in a hospital. In a review covering 12 nosocomial outbreaks in hospitals, an infection prevalence of up to $50 \%$ among patients on an affected ward was reported (Salgado et al., 2002). Sartor and colleagues (2002) found that $41 \%$ of patients and $23 \%$ of HCWs contracted influenza during a nosocomial outbreak on an internal medicine ward. This resulted in 14 missed working days among the $25 \mathrm{HCWs}$ on the affected ward, eight scheduled admissions had to be postponed and all emergency admissions had to be postponed for eleven days. In this context, applying influenza vaccination is the most effective method to prevent transmission of nosocomial influenza (CDC, 2010; Dolan, Iredale, Williams, \& Ameen, 2012), and studies show that it helps to reduce morbidity and mortality among patients (Ahmed et al., 2014; Amodio et al., 2014; Burls et al., 2006; Nichol et al., 1995; Thomas, Jefferson, \& Lasserson, 2010). A study in all eight Dutch University Medical Centers (UMCs) showed that an increase of $10.8 \%$ in the influenza vaccination uptake of HCWs by means of a multi-faceted campaign led to $6 \%$ less patients with hospital-acquired influenza and/or pneumonia in comparison with control hospitals (Riphagen-Dalhuisen et al., 2013). 
Secondly, unvaccinated HCWs have a more than three times higher risk to contract influenza, because of the patients they work with and because of the fast spreading that is characteristic for workplaces like hospitals (Kuster et al., 2011). It is estimated that $20 \%$ of HCWs get infected with influenza annually (Sartor et al., 2002). Many of them keep working despite being symptomatic and thereby facilitate the spreading of influenza (Ablah et al., 2008; Weingarten, Riedinger, Burnes Bolton, Miles, \& Ault, 1989). Moreover, a study from the UK demonstrated that of a sample of 518 unvaccinated HCWs, 120 (23\%) tested antibody positive to influenza, of which 71 (59\%) could not recall having had influenza (Elder, O’Donnell, McCruden, Symington, \& Carman, 1996). This shows that there might be a high incidence of non-symptomatic influenza among HCWs, who will also keep working and can thereby still act as vectors in the transmission of influenza to patients and colleagues.

Thirdly, studies have shown that vaccinating HCWs is cost-effective. Diseaseassociated costs can be calculated by considering the direct, indirect and intangible costs caused by a disease. Direct costs include medical treatments for the disease and hospitalizations, indirect costs amongst others result from work-absenteeism and loss of productivity, and intangible costs relate to the decline in quality of life (Commission of the European Communities, 2009). Sartor and colleagues (2002) had found that during a nosocomial outbreak in a hospital in France, direct costs associated with the outbreak were close to $€ 25.000$, which was an approximately $€ 3.000$ per infected patient. Other studies demonstrated the cost-effectiveness of vaccinating HCWs and it was estimated that up to $€ 43$ can be saved per person vaccinated (Burls et al., 2006; Nichol et al., 1995). In a council recommendation on seasonal influenza vaccination by the Commission of the European Communities, the total economic burden of influenza for industrialized countries, combining direct and indirect costs, was estimated at 56.7 million Euros per million people (Commission of the European Communities, 2009). Preaud and colleagues (2014) calculated the annual economic benefits of vaccinating $75 \%$ of the target groups, including HCWs, as stated in the EU Council Recommendation on seasonal influenza vaccination (2009) to be between $€ 190$ to $€ 226$ million over currently averted costs in Europe. Moreover, a study by Molinari and colleagues (2007) demonstrated that hospitalization costs associated with influenza was an important contributor to the total costs, however the indirect costs through work-absenteeism and lost lives represented the majority of the total economic burden of influenza.

\section{Low vaccination rates among European health care workers}

Despite the evidence for the effectiveness of vaccination in the prevention of nosocomial infections, in protecting HCWs, and saving health care costs, influenza vaccination coverage rates are low in Europe (Blank et al., 2009). Blank and colleagues (2009) reported vaccination rates of between 6.4 and $26.3 \%$ among HCWs from eleven European countries. Knowledge about the reasons why vaccination rates in HCWs are low is 
limited to self-reports of HCWs by means of cross-sectional surveys, which are rarely based on theories. The most often reported reasons to not get vaccinated are a low risk perception to contract influenza, influenza is not seen as a serious disease, and people are afraid of side-effects and/or long-term consequences from the vaccine, but also that it might cause influenza (Cohen \& Casken, 2012; Hofmann, Ferracin, Marsh, \& Dumas, 2006; Hollmeyer, Hayden, Poland, \& Buchholz, 2009). Moreover, HCWs reported to not believe in the relevance and scientific evidence of the vaccine in the prevention of influenza and the transmission to patients. Thus, it seems that there is a clear lack of knowledge about, and the belief in the necessity and effectiveness of influenza vaccination. Less often reported reasons that are responsible for vaccine refusal were self-perceived contraindications, such as pregnancy, fear of needles, and a general avoidance of medication (Hollmeyer et al., 2009). Organizational barriers such as time constraints, a lack of availability of vaccines, and inconvenience of locations for vaccination have also been reported by HCWs (Hofmann et al., 2006).

The most often reported reasons in favor of getting vaccinated against influenza were realistic assessments of the potential benefits of vaccination, such as selfprotection, patient protection, and the protection of family and friends, as well as the belief in the effectiveness of influenza vaccination, feelings of a professional responsibility to protect patients, and that it was recommended (Cohen \& Casken, 2012; Hakim, Gauer, \& McCullers 2011; Hofmann et al., 2006; Hollmeyer et al., 2009; Van den Dool et al., 2008). Organizational factors, such as easy access to the vaccine and the offer of free vaccines additionally facilitated vaccination (Cohen \& Casken, 2012; Hakim et al., 2011; Hofmann et al., 2006; Hollmeyer et al., 2009). The associated predictors of vaccination uptake have been shown to be a positive attitude towards influenza vaccination, positive social norms, having a good knowledge about influenza and vaccination, and previous vaccination uptake (Looijmans-van den Akker et al., 2009; Takayanagi, Cardoso, Costa, Araya, \& Machado, 2007; Zhang, While, \& Norman, 2011). Demographic variables that made vaccination more likely have been shown to be older age, having a chronic disease, working in health care for over 15 years, and being a physician compared to nursing staff (Cohen \& Casken, 2012; Looijmans-van den Akker et al., 2009; Takayanagi et al., 2007; Wicker, Rabenau, Doerr, \& Allwinn, 2009).

\section{The influence of media on mistrust towards vaccinations}

In order to gain more insight into the public's opinion towards vaccination and to foresee unrest that might lead to lower vaccination coverage rates, researchers and Health Authorities have suggested the monitoring of the Internet (Betsch et al., 2012; Harmsen, 2014; Kok et al., 2011; McNab, 2009). That way, changes in the public's opinion can be detected and possibly even reacted upon. Interestingly, Gesser-Edelsburg, Walter, and Green (2014) pointed out that health care professionals might react similarly to health information as the general public when it personally concerns them, as in the 
decision whether to get vaccinated against influenza and they might therefore react similarly to the media coverage.

The Internet has become an important source for accessing health information. In fact, more than $50 \%$ of Internet users search for health information online (Eysenbach \& Köhler, 2004; Fox, 2011; Kummervold et al., 2008). It has been suggested that the Internet much more critically evaluates topics such as vaccination, compared with traditional media (Zimmerman et al., 2005). Anti-vaccination lobbying is wide-spread on the Internet and it has been shown to increase perceived risk of vaccinations and to decrease the intention to get vaccinated (Betsch, Renkewitz, Betsch, \& Ulshöfer, 2010). There are several developments that explain why influenza vaccination is critically discussed in the media. In 2011, a debate about the effectiveness of influenza vaccination in the Netherlands originated from a public statement of a critical general practitioner and influenza vaccination opponent. He disputed the effectiveness of influenza vaccination and additionally claimed that the experts at the National Institute for Public Health and the Environment (RIVM) responsible for the national vaccination recommendations are not independent in their advice because of their collaboration with the pharmaceutical industry (Gebu, 2011). The RIVM and the Dutch government thereupon started a court case against the general practitioner in order to restore their credibility. The general practitioner, who felt restricted in his freedom of speech, received a lot of support from the medical field as well as the media. The court ruled that the general practitioner's behavior was not illegal, but that he unrightfully alleged experts at the RIVM to have a conflict of interest when they advise on national recommendations about vaccination.

The statements by the general practitioner were amongst others based on a systematic review by the Cochrane Collaboration, a non-governmental, non-profit organization which conducts systematic reviews of medical research information for recommendations about health care interventions (Jefferson et al., 2010). The Cochrane Collaboration enjoys wide acceptance in the medical field and has a seat in the World Health Assembly since 2011. In its 2010 review about the effectiveness of influenza vaccination for the protection of the elderly, it was concluded that there is no evidence supporting the health care intervention of vaccinating the elderly (Gebu, 2011; Jefferson et al., 2010). Beyer and colleagues (2013) repeated the review with the same data and they came to a very different conclusion; that influenza vaccination does reduce the risk of influenza infection, related diseases and death among the elderly and should be maintained as a preventive measure.

Media coverage on controversies about other vaccinations is also likely to have an influence on the opinion towards influenza vaccination and the decision whether to get vaccinated. For example, in 2009, when the world faced the threat of the influenza A (H1N1) pandemic, also known as Mexican flu, the Dutch government bought 34 million doses of the pandemic vaccine and started a large media campaign, advising all people to get vaccinated (Van der Sande et al., 2012). However, the Mexican flu out- 
break resulted into a mild epidemic and 20 million vaccines had to be destroyed. This resulted in a national discussion about the government's decision, the role of experts, as well as the fear that was generated by national media reports (Bijl \& Schellekens, 2011; Vasterman \& Ruigrok, 2013). Additionally, a Finnish study had claimed that the Mexican flu vaccination Pandemrix causes narcolepsy in children (Partinen et al., 2012), which was not confirmed (Melén et al., 2013).

A similar discussion as the one about the Mexican flu exists about the annual call for adolescent girls aged 12 years and older to get vaccinated against the human papilloma virus (HPV) that is believed to cause cervical cancer. Contradictory scientific findings about the safety and effectiveness of the vaccine lead some people to believe that the vaccination campaign is just another source of money for the pharmaceutical industry (Rondy, van Lier, van de Kassteele, Rust, \& de Melker, 2010). This might further contribute to an increase in the mistrust of the population towards vaccinations in general.

Another example of a refuted scientific finding that nonetheless seems to influence people's opinions about vaccinations is the suggested link between the measles, mumps, rubella vaccine and the development of autism in children (Wakefield et al., 1998). Although retracted, the article is still the cause for a lower vaccination uptake in the U.K.'s National Immunization Program (Brown et al., 2012; The Lancet, 2010).

\section{Attempts to increase influenza vaccination uptake among health care workers}

Recommendations from Health Authorities are clear and most hospitals as well as other health care facilities offer influenza vaccination for free and on work site for their HCWs. However, this is clearly not enough to convince HCWs to get vaccinated (Palache, 2011). This is why a number of interventions have been developed in recent years to increase the vaccination coverage rate in this group.

The most commonly used elements in interventions to increase the influenza vaccination uptake in $\mathrm{HCW}$ s are education and the reduction of practical barriers to vaccination (Chittaro et al., 2009; Cooper \& O'Reilly, 2002; Llupià et al., 2010; Llupià et al., 2013; Riphagen-Dalhuisen et al., 2013; Salgado, Giannetta, Hayden, \& Farr, 2004). Education includes factual information about influenza, the risks and benefits of vaccination, and the importance of vaccinating HCWs to prevent the transmission of influenza to vulnerable patients. It focuses mainly on the determinants attitude, knowledge and risk perception and has been implemented using interpersonal means (i.e. staff meetings and discussions), several different print media (i.e. posters, brochures, letters, leaflets), as well as electronic media (i.e. videos, websites, intranet) (Chittaro et al., 2009; Cooper \& O’Reilly, 2002; Doratotaj, Macknin, \& Worley, 2008; Llupià et al., 2010; Llupià et al., 2013; Riphagen-Dalhuisen et al., 2013; Salgado et al., 2004). Reducing practical barriers and increasing access, next to offering the vaccine for free and on work site, has been done by extending the times and locations for vaccination, by in- 
creasing the amount of staff who apply vaccination, and by introducing mobile carts that allow for vaccination directly on the wards (Chittaro et al., 2009; Cooper \& O’Reilly, 2002; Llupià et al., 2010; Llupià et al., 2013; Riphagen-Dalhuisen et al., 2013; Salgado et al., 2004; Sartor et al., 2004). Another intervention element that has been used several times before is the use of social norms and role models. Working with leadership support, vaccination champions on the wards, and publishing names or showing photos/videos of vaccinated staff is supposed to present HCWs with positive social norms towards vaccination (Hood \& Smith, 2009; Llupià et al., 2010; Llupià et al., 2013; Riphagen-Dalhuisen et al., 2013; Sartor et al., 2004). Moreover, a Swiss and a Dutch study introduced the use of badges for HCWs who got vaccinated ("I am vaccinated against influenza to protect you"; "Consciously vaccinated for you."), and in the Swiss study even for the unvaccinated HCWs ("I wear a mask to protect you.") (Iten, Bonfillon, Bouvard, Siegrist, \& Pittet, 2013; Riphagen-Dalhuisen et al., 2013). Some interventions have additionally worked with incentives to promote influenza vaccination, including raffle tickets to win meals, weekend trips, vacations, I pods, laptops, and the donation of $€ 1$ per vaccinated HCW to a good cause (Ajenjo, Woeltje, Babock, Gemeinhart, \& Jones, 2010; Doratotaj et al., 2008; Llupià et al., 2010; Llupià et al., 2013).

Unfortunately, existing programs show at most moderate effects. Lam, Chambers, Pierrynowski MacDougall, and McCarthy (2010) also concluded from their systematic review, including seven studies in hospital settings, that education, promotion, and improved access to influenza vaccination show only small effects on the vaccination uptake of HCWs. Only campaigns that included regulations, such as mandatory declination forms and the requirement of wearing masks for unvaccinated staff, showed higher rates in uptake (Lam et al., 2010). However, implementing regulations that could be perceived as obligations to get vaccinated against influenza are very uncommon in Europe and will most likely meet resistance.

Effects on vaccination uptake rates are difficult to compare, because of the different designs and time periods that have been used, and very different baseline coverage rates. However, it is noticeable that vaccination rates post intervention are at a maximum of $37 \%$ in European settings (Llupià et al., 2010), while they reach $84 \%$ in the United States (Hood \& Smith, 2009), not considering the interventions that installed mandatory vaccinations.

Theory- and evidence-based approaches can be expected to have the highest potential for accomplishing behavior change. Intervention Mapping (IM) (Bartholomew, Parcel, Kok, Gottlieb, \& Fernandez, 2011) describes an iterative planning process, in which theory- and evidence-based health promotion programs are developed systematically by means of six steps. In step 1, the Needs Assessment is conducted in which the problem is analyzed combining theory and evidence. In step 2, the program objectives or behavioral goals are established. Appropriate theory-based methods and practical applications are chosen in step 3. Steps 4 and 5 include the planning of the 
program development and the implementation of that program. Finally, step 6 entails the program evaluation, which will most likely lead to a revision of the program (Bartholomew et al., 2011). Kok and colleagues (2011) proposed to apply IM to the development of interventions to promote the influenza vaccination uptake of HCWs, parts of which will be described in this cumulative dissertation.

\section{Dissertation outline}

The studies described in this dissertation were performed to gain insight into the drivers of HCWs' decision whether to get vaccinated against influenza, in order to develop a theory- and evidence-based health promotion program to increase the influenza vaccination uptake among this group.

Before investigating the reasons and social cognitive predictors of HCWs' decision to get vaccinated against influenza, the first two chapters look at the opinion of the general public and pre-clinical medical students towards influenza and vaccination. In order to understand the communication sentiment about influenza vaccination in the general public, chapter $\mathbf{2}$ describes a study in which we monitored the online communication about influenza and influenza vaccination on news sites and social media websites in the month before, during, and after the influenza epidemic 2012 occurred in the Netherlands.

Past research clearly shows the hesitance of HCWs to get vaccinated against influenza. However, it is not clear whether HCWs form this negative opinion during their clinical careers or whether it exists earlier, for instance during medical training. There are only very few studies that include medical students and no study that explored the social cognitive predictors of medical students' intention to get vaccinated against influenza. Therefore, we conducted a study investigating the social cognitive predictors of pre-clinical medical students' intention to get vaccinated against influenza, described in chapter 3.

Recent studies have almost exclusively used quantitative questionnaires in order to identify determinants of vaccination behavior. In these questionnaires, HCWs receive constructed reasons against and in favor of influenza vaccination and are forced to choose. This may lead HCWs to select answers even if they do not reflect their true reasons for (not) getting vaccinated. Chapter 4 presents the findings of a study exploring social cognitive predictors and the beliefs that underlie them by means of one-onone interviews with HCWs of hospitals in Belgium, Germany, and the Netherlands. Conducting the interviews in three neighboring European countries was meant to provide insight into similarities and differences in predictors that might have to be considered when developing health promoting programs to increase influenza vaccination uptake in health care settings.

In order to measure the relative and combined strength of the in chapter 4 identified social cognitive predictors and three additional beliefs in explaining the intention 


\section{Chapter 1}

to get vaccinated of Belgian, Dutch and German HCWs, we conducted an online quantitative study that is described in chapter $\mathbf{5}$.

Chapter 6 describes a survey study that shows the social cognitive variables that influence Dutch HCWs' motivation to get vaccinated against influenza, and whether their intention is a good predictor of their actual vaccination behavior.

Chapter 7 describes the implementation and effect evaluation of a behavior change intervention to increase the influenza vaccination uptake of Dutch HCWs in a tertiary care center of expertise for the diagnosis and treatment of patients with complex chronic organ failure.

Finally, chapter 8 discusses the major findings of the studies, describes the development and implementation of the proposed behavior change intervention and outlines practical implications and recommendations for future research. 


\section{CHAPTER 2}

\section{A qualitative study of the coverage of influenza vaccination on Dutch news sites and social media websites}

Published as:

Lehmann, B. A., Ruiter, R. A. C., \& Kok, G. (2013). A qualitative study of the coverage of influenza vaccination on Dutch news sites and social media websites. BMC Public Health, 13, 547. 


\section{ABSTRACT}

Information about influenza and the effectiveness of vaccination against influenza is largely available on the Internet, and may influence individual decision making about participation in future influenza vaccination rounds. E-health information has often been found to be inaccurate, or even to contradict Health Authority recommendations, especially when it concerns controversial topics. By means of an online media monitoring program, Dutch news sites and social media websites were scanned for the Dutch counterparts of the terms influenza, vaccination, vaccine and epidemic during February, March and April 2012. Data were processed with QSR NVivo 8.0 and analyzed using a general inductive approach. Three overarching themes were found in both media sources: (1) the (upcoming) influenza epidemic, (2) general information regarding the virus, its prevention and treatment, and (3) uncertainty and mistrust regarding influenza vaccination. Social media tended to report earlier on developments such as the occurrence of an influenza epidemic. The greatest difference was that in social media, influenza was not considered to be a serious disease, and more opposition to the flu shot was expressed in social media, as compared to news media. News media and social media discussed the same topics regarding influenza, but differed in message tone. Whereas news media reports tended to be more objective and nonjudgmental, social media more critically evaluated the harmfulness of influenza and the necessity of the flu shot. Media may influence decision making and behaviors of Internet users and may thereby influence the success of vaccination campaigns and recommendations made by Health Authorities. Social media may be more of a problem in this sense, since it is neither controlled nor censored. Future research should investigate the actual impact of Internet media on the influenza decision making process of its users. 


\section{INTRODUCTION}

The Internet is an important source for accessing health information. In fact, $55 \%$ of Internet users search for health information online (Brodie et al., 2000; Eysenbach \& Köhler, 2004; Hsu et al., 2004). The Internet is the largest and most easily accessible library in the world and enables users to find information in a time-saving way. By the year 2000, more than 70000 e-health websites existed (Grandinetti, 2000). E-health websites are websites that provide health-information for educational purposes, selfcare, and for the simplification of health-communication (Oh, Rizo, Enkin, \& Jadad, 2005). The Internet is the second most trusted source of health information, following the personal advice of one's own general practitioner (Dolan, Iredale, Williams, \& Ameen, 2004; Dumitru et al., 2007). However, concerns have been raised by medical professionals and Internet users about the quality and comprehensibility of health information, especially when it is directed at the broader public (Berland et al., 2001; Cline \& Haynes, 2001). That is, the Internet is an unregulated resource that not only enables anyone to access information, but also makes it possible for anyone to publish information (Dolan et al., 2004; Hardey, 2001). The information overload is enormous (Cline \& Haynes, 2001), which makes it increasingly difficult to ensure the credibility of health information sources. Users mostly use search engines like Google and Yahoo to access health websites. However, a study by Berland and colleagues (2001) shows that searches with a search engine require high reading abilities and often do not lead to websites with relevant content. Moreover, a number of different studies found website content did not adhere with official recommendations for prevention and treatment (Cline \& Haynes, 2001). For example, in a study about the reliability of websites that informed parents about home management of an ill child, only 4 out of 41 websites displayed information in accordance with official recommendations (Impicciatore, Pandolfini, Casella, \& Bonati, 1997).

After consulting health information on the Internet, people feel reassured twice as much as they feel anxious (Andreassen et al., 2007). This could mean that people select sources on the basis of what they already believe to be true. This also means that people who already distrust public health recommendations may be biased to read information that is given by providers holding the same attitude. In social psychology, this tendency of people to favor information that confirms their belief has been termed the confirmation bias, a bias which can occur unintentionally and without awareness (Nickerson, 1998).

\section{Social Media}

Social media refers to Internet content that is continuously modified by all collaborating users of a publicly accessible website instead of by professionals (Kaplan \& Haenlein, 2010). It offers easy ways for people to share and read information on a large 
scale. In contrast to social media websites, news sites refer to websites that generate original news written exclusively by registered members (e.g., editorial staff).

Social networking has increased rapidly in recent years, making Twitter and Facebook the most popular platforms for sharing and communicating information (Qualman, 2011). More than half of all Internet users joined a social networking site in 2009 (Wright \& Hinson, 2009). It has been found that people trust the opinions of their peers more than they trust the opinions of officials, which makes social networking sites increasingly powerful (Qualman, 2011; Schmitt-Beck, 2003). Individuals turn to each other for advice and trust that the experiences of their friends and acquaintances represent the truth (Qualman, 2011). It has been found that health information in online support groups and chat rooms is often inaccurate (Cline \& Haynes, 2001). Social networking sites, such as Twitter and Facebook may show a similar insufficiency regarding health information. There has been, however, no study as of yet investigating the content of health information on social networking sites.

The purpose of this study is to explore the content of health information regarding influenza vaccination in the month before, during, and after an influenza epidemic occurred in the Netherlands. Cline and Haynes (2001) have suggested that using the Internet for accessing health information is dangerous for already controversial topics. Influenza vaccination is a highly controversial issue in the Netherlands that is heavily discussed in the news media and also in the social media.

Objectivity is not always guaranteed and this might especially be true for social media platforms in which no censoring takes place. This could in turn negatively influence the decision making and behavior of Internet users towards influenza vaccination and thereby may influence the success of Health Authority recommendations with regard to influenza vaccination. Therefore, the purpose of this study is to describe the news site and social media website content about influenza vaccination on the Internet, as well as the similarities and differences between these two types of media content.

\section{METHODS}

\section{Data collection}

In this article, we describe the Dutch Internet content of news sites and social media websites that became accessible in the month before, during, and after the 2012 influenza epidemic occurred. We retrospectively selected the three months February (before), March (during) and April (after), based on official documentations of influenza activity in the Netherlands by the Netherlands Institute for Health Services Research (NIVEL). 
Data were collected using Clipit, an online media monitoring program that can be used to search the Internet for preselected terms. In the case of this study, the search terms influenza, vaccination, vaccine and epidemic were monitored daily in more than 10.000 Dutch Internet sources, including news sites and press reports, discussion forums, weblogs, newsletters, reviews, as well as social media websites. A search profile with the aforementioned terms was already activated in June 2011 by the National Institute for Public Health and the Environment (RIVM).

News websites, as defined by Clipit, range from print media that is additionally represented on the Internet to more unconventional websites for members of groups that share certain beliefs, such as the belief that vaccinations are harmful. Social media websites monitored by Clipit were Twitter, Facebook, Linkedin and Hyves. Hyves is the Dutch equivalent to Facebook. In the case of social networking sites, only reports that were made publicly accessible by their authors were detected by the monitoring program. Clipit continuously provided links to websites in which one or more of the above mentioned search terms were used. These links were individually opened and the content of the appearing Internet site was then copied and transferred to Word documents. Only the first page that appeared when opening the link was taken into consideration and read in its entirety. No further links were opened, thus restricting the level of analysis to the primary source. Data collection and analysis was performed by the first author.

\section{Data analysis}

The Internet content was processed with QSR NVivo 8.0 (Doncaster, Australia). The content analysis was based on a general inductive approach (Thomas, 2006) and conducted by a single coder. Through detailed reading of the raw data, themes were identified and data were subsequently categorized under separate headings (called nodes in QSR NVivo 8.0). Afterwards, categories were linked to one another, which led to the identification of overarching themes relevant for the description of online content regarding influenza as a whole. This process was repeated separately for each month and for the two different kinds of online media sources (news sites and social media websites). Exclusion criteria were website content about influenza-related topics concerning countries other than the Netherlands (including the Dutch speaking part of Belgium) and website content about the bird flu. Additionally, themes on news sites and in social media posts that only occurred once, or showed no consistency with more often reported themes were excluded to reduce the overload of information. Following analysis, quotes were selected on the basis of their representativeness for the findings and were subsequently translated from Dutch into English. 


\section{RESULTS}

In February, March and April 2012, three different overarching themes were consistently identified in the online content of news sites and social media websites: (1) the (upcoming) influenza epidemic, (2) general information regarding the virus, its prevention and treatment, and (3) the uncertainty and mistrust regarding influenza vaccination in the Netherlands. The information is summarized below according to these three themes and per month. In total, 3553 of the 4441 reports that were detected by Clipit were included in the results: 1305 reports ( $N=204$ on news websites; $N=1101$ on social media websites) in February, 1527 reports ( $N=276$ on news websites; $N=1251$ on social media websites) in March and 721 reports ( $N=61$ on news websites; $N=660$ on social media websites) in April (see Table 2.1). See Table 2.2 for an overview of the information that is summarized below.

Table 2.1 Number of included and excluded influenza reports for each month and total

\begin{tabular}{|c|c|c|c|c|c|c|c|}
\hline \multirow[b]{2}{*}{ Month } & \multirow{2}{*}{$\begin{array}{l}\text { Total reports } \\
\text { Total (\%) }\end{array}$} & \multicolumn{3}{|c|}{ News media } & \multicolumn{3}{|c|}{ Social media } \\
\hline & & Total & Incl. (\%) & Excl. (\%) & Total & Incl. (\%) & Excl. (\%) \\
\hline Reports detected & 4441 & 939 & $541(58)$ & $398(42)$ & 3502 & $3012(86)$ & $490(14)$ \\
\hline February & $1574(35)$ & 299 & $204(68)$ & $95(32)$ & 1275 & $1101(86)$ & $174(14)$ \\
\hline March & $1992(45)$ & 480 & $276(58)$ & $204(42)$ & 1512 & $1251(83)$ & 261 (17) \\
\hline April & $875(20)$ & 160 & $61(38)$ & $99(62)$ & 715 & $660(92)$ & $55(8)$ \\
\hline
\end{tabular}

\section{News websites - February 2012}

\section{Upcoming epidemic}

A number of news sites reported about the fact that influenza activity was low in the Netherlands and that epidemics usually occur in December or January. Some articles discussed the possibility that 2012 might be a year without an influenza epidemic:

"It is possible that the flu won't peak until the end of February. That is the same time that primary schools have vacation and many people will go on a winter holiday, which increases the chance that the flu may even pass us by this year." (www.nursing.nl) 
Table 2.2 Summary of website content by theme, month and source

\begin{tabular}{|c|c|c|c|}
\hline Month & Epidemic & $\begin{array}{l}\text { Information, prevention \& } \\
\text { treatment }\end{array}$ & Uncertainty \& Mistrust \\
\hline \multicolumn{4}{|c|}{ News Sites \& Press } \\
\hline $\begin{array}{l}\text { Febr } \\
2012\end{array}$ & $\begin{array}{l}\text { Influenza activity low; } \\
\text { increase in other European } \\
\text { countries; criteria for } \\
\text { epidemic; identifying the } \\
\text { type of influenza. }\end{array}$ & $\begin{array}{l}\text { Difference between flu and } \\
\text { common cold; symptoms of } \\
\text { flu; possible complications of } \\
\text { an infection; variants of virus; } \\
\text { severity of influenza; health } \\
\text { effects of vaccination; } \\
\text { vaccinated women and higher } \\
\text { birth weight; prevention and } \\
\text { treatment. }\end{array}$ & $\begin{array}{l}\text { Uncertainty about effectiveness } \\
\text { of vaccination; difficulty of } \\
\text { proving effectiveness; } \\
\text { collaboration between science, } \\
\text { politics and pharmaceutical } \\
\text { industry; dangerous effects of } \\
\text { vaccination; spreading illness; } \\
\text { narcolepsy as effect of the } \\
\text { pandemic (H1N1) vaccine in } \\
2009 .\end{array}$ \\
\hline $\begin{array}{l}\text { March } \\
2012\end{array}$ & $\begin{array}{l}\text { Epidemic in south of the } \\
\text { Netherlands; presence of } \\
\text { epidemic; recognition of the } \\
\text { flu; effectiveness of flu shot; } \\
\text { complications for risk } \\
\text { groups; decreasing influenza } \\
\text { activity. }\end{array}$ & $\begin{array}{l}\text { Only } 70 \% \text { of employees stayed } \\
\text { at home when sick, possibly } \\
\text { because of the economic crisis; } \\
\text { information about virus and } \\
\text { vaccine; Google flu; } \\
\text { prevention, treatment and } \\
\text { vaccination as the only } \\
\text { effective protection. }\end{array}$ & $\begin{array}{l}\text { Narcolepsy; collaboration } \\
\text { between science and the } \\
\text { pharmaceutical industry. }\end{array}$ \\
\hline $\begin{array}{l}\text { April } \\
2012\end{array}$ & $\begin{array}{l}\text { End of epidemic; criteria for } \\
\text { epidemic identification. }\end{array}$ & $\begin{array}{l}\text { One nursing home with } 30 \% \\
\text { infections. }\end{array}$ & $\begin{array}{l}\text { Narcolepsy; loss of vaccines as a } \\
\text { result of inaccurate storage. }\end{array}$ \\
\hline \multicolumn{4}{|c|}{ Social Media } \\
\hline $\begin{array}{l}\text { Febr } \\
2012\end{array}$ & $\begin{array}{l}\text { South of Netherlands closer } \\
\text { to epidemic threshold (links } \\
\text { to news sites); epidemic } \\
\text { present (user experiences/ } \\
\text { perceptions). }\end{array}$ & $\begin{array}{l}\text { Difference between stomach } \\
\text { flu and seasonal flu, viral and } \\
\text { bacterial infection; prevention } \\
\text { (e.g. vitamin C\&D); } \\
\text { effectiveness flu shot (user } \\
\text { experience). }\end{array}$ & $\begin{array}{l}\text { Uncertainty about effectiveness } \\
\text { of vaccination; ineffectiveness } \\
\text { flu shot (user experience + links } \\
\text { to websites); advice against } \\
\text { vaccination. }\end{array}$ \\
\hline $\begin{array}{l}\text { March } \\
2012\end{array}$ & $\begin{array}{l}\text { Late onset epidemic (links to } \\
\text { news sites); presence of } \\
\text { epidemic (links to news sites } \\
\text { + user experience). }\end{array}$ & $\begin{array}{l}40 \% \text { Dutch people have flu } \\
\text { each year; less flu-related } \\
\text { absenteeism because of } \\
\text { economic crisis; work overload } \\
\text { in hospitals during epidemic; } \\
\text { vitamin D metabolism and flu; } \\
\text { prevention; effectiveness flu } \\
\text { shot (own experience). }\end{array}$ & $\begin{array}{l}\text { Possibility of getting flu despite } \\
\text { flu shot; economic background } \\
\text { vaccination; ineffectiveness of } \\
\text { flu shot; dangerousness of flu } \\
\text { shot (e.g. Alzheimer's, } \\
\text { narcolepsy); collaboration } \\
\text { between science and } \\
\text { pharmaceutical industry. }\end{array}$ \\
\hline $\begin{array}{l}\text { April } \\
2012\end{array}$ & $\begin{array}{l}\text { Epidemic present (own } \\
\text { experience); end of epidemic } \\
\text { (links to news sites). }\end{array}$ & $\begin{array}{l}\text { Numbers of deaths epidemic } \\
2012 \text { (link to website); } \\
\text { decreasing work-absenteeism; } \\
\text { effectiveness of flu shot (own } \\
\text { experience). }\end{array}$ & $\begin{array}{l}\text { Possibility of getting flu despite } \\
\text { flu shot; ineffectiveness + } \\
\text { dangerousness of flu shot; } \\
\text { hygiene in workplace as means } \\
\text { of prevention; discussion about } \\
\text { loss of vaccines as a result of } \\
\text { inaccurate storage. }\end{array}$ \\
\hline
\end{tabular}


In the beginning of February 2012, reports showed that most European neighbors experienced similar levels of influenza activity. However, in the middle of February, news sites reported an increase in influenza activity in the south of Europe. This resulted in an increase of news reports about the threat of an upcoming epidemic in the Netherlands.

"In the past weeks, flu became more active in more and more countries around us; In South and South-East Europe, as well as in Norway. Meanwhile, the flu reached Belgium, but did not yet reach Germany, Britain and Denmark." (www.gezondheidskrant.nl)

Next to information about the development of influenza activity, news sites also informed readers about the process by which influenza epidemics are identified:

"NIVEL (Netherlands Institute for Health Services Research) speaks about an epidemic if within two successive weeks more than 51 per 100.000 people with flu-like conditions are reported and virological research reveals the virus in nose- and throat samples." (gezondheid.blog.nl)

Other reports discussed the type of virus that was expected in 2012:

"[...] it is mainly influenza B and influenza AH3N2. Both flu-variants are covered by the vaccine that was offered for high risk groups in the winter season. The AH1N1-virus that caused the pandemic in 2009 is rarely seen." (medischcontact.artsennet.nl)

Several times it was indicated in news site reports that there was no apparent reason for a late influenza epidemic.

\section{Information about the virus, prevention and treatment}

Next to information about the expected upcoming influenza epidemic, readers were informed about the differences between the flu and a common cold, symptoms of the flu, possible complications, especially for high risk groups, and the effect of influenza vaccination. The mutations that occur in the influenza virus were outlined in several articles as a means to explain why annual vaccination is required:

"No single influenza virus is the same. Every year it is a different virus. You can get the flu every year. Therefore, the vaccine has a different composition every time. The composition is matched to the viruses that are expected to be present the following winter. Therefore, it is necessary to get vaccinated every year." (mens-en-gezondheid.infonu.nl)

A number of reports emphasized the severity and consequences of influenza: 
"The flu is the most underestimated illness that exists." (www.healthylives.nl)

"Every year approximately 820.000 Dutch people get the flu. During an average influenza epidemic in the winter, 250 to 2.000 people in the Netherlands die because of the flu or complications of the flu. Victims mainly belong to the high risk groups." (www.nivel.nl)

Recent research was also discussed on news sites. For example, a study was reported describing the finding that mothers who got vaccinated against influenza while they were pregnant delivered babies with a higher birth weight than did mothers who did not get vaccinated against influenza (Steinhoff et al., 2012).

Next to general information, means of prevention and treatment of an influenza infection were described. Some articles dealt with homeopathic means to prevent and treat influenza but also indicated that this is an additional tool next to vaccination. For example:

"People that got vaccinated in autumn are protected, but what can people do that did not get vaccinated to protect themselves? [...] For people that want to protect themselves but dread getting a flu shot, there is Polyinfluenzium." (www.nieuwslog.nl)

Other reports discussed ways of keeping or acquiring a good physical resistance, such as drinking a lot of water, wearing warm clothes, eating healthily, ensuring good hand hygiene, and performing outdoor physical activities.

\section{Uncertainty \& mistrust}

News sites also dealt with uncertainty and mistrust regarding science. In particular, the uncertainty surrounding whether influenza vaccination is effective or possibly even dangerous was discussed. One report about the 'Evidence Beast' summarized the debate that was then described in the content of a number of news sites that were published online in February. It deals with the difficulty in proving that the morbidity and mortality rate would be much higher if influenza vaccination did not exist or had not been effective.

"We have to prove that by doing something good, something bad will not happen, which would have happened if we had not done anything. Sounds complicated and that is what it is. [...] We have learned this from the flu vaccination incident." (www.skipr.nl)

Some news reports discussed the developments in the Netherlands with regard to the views that the general population hold about science. One issue is that a proportion of the general public does not see science as an independent and reliable source any- 
more, when it might have direct consequences for their own lives. People are said to fear that science is biased by politics, as well as by the pharmaceutical industry.

"Citizens don't just accept the 'expert stories', even more so, when they have direct consequences for their lives. When scientists and politics get too close, problems can arise [...]." (www.volkskrant.nl)

There were several articles reporting about the dangerous effects that influenza vaccination might have. These reports provide assumed evidence for the fatal effects of vaccination. Individual ingredients and their effect on one another are described. Supposed "experts" are cited and speak about secret plans of the government to deliberately infect people with a virus through the vaccine in order to create an elite or to control the growth of the world's population.

"Recently, it (flu vaccination) is used more and more to spread illness. That is the reason why most people who get the flu in winter are the same people who get the flu shot regularly." (www.argusoog.org)

Other articles strengthened their point of view by referring to the increased incidences of narcolepsy in children and its link to the pandemic (H1N1) vaccination in 2009, which was first suggested by a Finnish study (Partinen et al., 2012). Readers are asked to be more critical about vaccinations and to propagate their opinion to other people in order to prevent fatal consequences.

"We hope that you, the reader of this article, will do your best to NOT keep quiet. That during gatherings, such as parties and dinners you will NOT avoid this topic, but instead try to convince people that vaccines are NOT safe. It is a FACT!! There are enough scientists who strongly doubt the necessity of vaccines." (www.wanttoknow.nl)

\section{News websites - March 2012}

\section{(Upcoming) epidemic}

In the beginning of March, several news sites reported that there was an on-going epidemic in the south of the Netherlands:

"The Netherlands did manage to avoid the flu for a long period this winter. In the south of the Netherlands, however, there has been an epidemic since March 1st." (www.dichtbij.nl, www.omroepzeeland.nl, www.drimble.nl)

It was also indicated that the chance for the north of the Netherlands to experience an epidemic had increased. More people were identified with the influenza virus and 
schools were about to open again after the vacation. However, in the north of the Netherlands, activity was still below the epidemic threshold.

"In the week of February 27th until March 4th, on average 50 per 100.000 Dutch people reported having flu-like complaints to their general practitioner. [...] Last week it was 47. We are actually very close to the epidemic threshold." (gezondheid.blog.nl)

After the first week of March, news sites mostly reported that the epidemic was now in fact happening in the Netherlands.

"In the beginning of March 2012, the Netherlands did experience an influenza epidemic after all. More than 78 per 100.000 inhabitants went to the general practitioner with flu-like complaints. For a start it is a mild epidemic. Most people get better after 3-5 days - says NIVEL, the Netherlands Institute for Health Services Research." (www.amstelveenweb.com)

Several articles additionally provided information about how to recognize whether someone was actually infected with the influenza virus. A few articles also referred to the wider impact of an influenza epidemic and the effectiveness of the flu shot.

"The flu is an illness that will go away by itself, so people don't have to worry. [...] The flu shot provides you with approximately 70 percent protection against the flu. People who get the flu shot and get ill anyway are still better protected against complications from the virus." (Donker, NIVEL, www.nrc.nl, www.AD.nl, www.volkskrant.nl, www.gezondheidsnet.nl)

It was also mentioned that young children, aged $0-4$ years of age, as well as the elderly, aged 65 years of age and older, are at highest risk to suffer from complications of an influenza infection. In the fourth week of March, news sites started to report about the end of the epidemic as influenza activity decreased again.

"The influenza epidemic - as far as you can call it such - seems to already be on its way out." (gezondheid.blog.nl)

Influenza activity in the Netherlands was said to be comparable to the activity in Europe with regard to the time of occurrence, as well as the type of virus that was detected.

\section{Information about the virus, prevention and treatment}

The most often reported news regarding general information about influenza in March was the discovery that in the past winter only $70 \%$ of employees decided to stay at home when ill with flu instead of going to work. Readers were advised to stay at home. 
"Real flu is not to be taken too lightly. Sometimes complications can emerge, such as a sore throat, pneumonia, meningitis, inflammation of the myocardial muscle, as well as additional bacterial infections. [...] Therefore, it is important to recover completely." (www.ffm.nl)

The economic crisis was seen as a possible reason for this phenomenon. Next to these news items, there were also some articles with general information about the virus and the process by which the vaccine works. Other articles dealt with the idea of using Google searches in order to predict epidemics, a project called Google Flu.

Discussions regarding means of prevention and treatment of influenza were similar to those reported in February. Readers were informed how to improve their health status with rest and enough sleep, a lot of water, healthy food and outdoor physical activity. As a means of prevention, a number of articles stated that the flu shot was the only effective protection. This was also the conclusion of an article that examined the effectiveness of other flu medication.

"According to pharmacist $X$, those medications (to treat flu complaints) do not help to prevent someone from getting the flu. [...] The only proved, effective method of prevention is vaccination." (www.rtl.nl)

\section{Uncertainty and mistrust}

As in February, in March there were a number of articles concerned with the finding that narcolepsy was found more often in children after the pandemic (H1N1) vaccination in 2009. Furthermore, debates about the trust in science and the collaboration between scientists and the pharmaceutical industry were again discussed in online news. Consequences of disabling the interaction between science and the industry were discussed.

"Well yes, maybe the paid experts are too positive about medication, but you could also say that medical practitioners who do not collaborate with the industry do not do so because they are too negative about medication. [...] If we have the illusion that medicine gets better if we stop the interactions, [...] then there will simply not be any new medication." (www.artsennet.nl)

\section{News websites - April 2012}

\section{End of epidemic}

In April 2012, several news sites reported that the epidemic of March had already ended. 
"The influenza epidemic was short and not even strong. In the beginning of this year, the flu stayed for some weeks beneath the threshold of an epidemic. Not until the beginning of March did the flu start to spread. However, the peak only lasted one week." (www.nerderland-davos.nl)

Readers were again informed about the criteria that are used to identify an epidemic.

\section{Information about the virus, prevention and treatment}

Some news sites reported about a nursing home in which 45 of the 160 residents had had a serious influenza infection, 12 of whom had to be hospitalized. However, the situation was resolved after a few days and further transmission was stopped.

"The flu breakout put a lot of pressure on the care in the (nursing home). Director X: 'Suddenly, a quarter of the residents got ill. The organization was in chaos for a short while, because a lot of extra care had to be given. [...]'." (www.hogeveen.nu)

\section{Uncertainty and mistrust}

Most articles dealing with uncertainty that were published in April discussed the finding that there was now more evidence that narcolepsy is caused by Pandemrix, the vaccine used for the pandemic (H1N1) in 2009, as well as a news report about an employee of the National Vaccine Institute of the Netherlands that had left those vaccines out of the refrigerator, at great financial cost.

"THE HAGUE - Pandemic (H1N1) 2009 vaccines that could have been sold abroad, were made useless by a blunder of an employee of the National Vaccine Institute (NVI) in Bilthoven." (www.rtl.nl)

\section{Social media websites - February 2012}

\section{(Upcoming) epidemic}

In the beginning of February 2012, posts on social networking sites mostly reported that influenza activity was still low and that there was still no epidemic present in the Netherlands. However, it was reported that the south of the country was getting closer to epidemic levels. Several posts on Twitter provided a link to corresponding news site reports.

"The flu epidemic is not yet present in the Netherlands, but it seems to be coming. (link to news site)" (Twitter) 
In February, there were also already posts that an epidemic had arrived in the Netherlands. People who put the posts on Twitter referred to their own experience and news they had read, without providing the corresponding article.

"The flu epidemic is a fact: Half of my floor is ill including myself \#stomach flu" (Twitter)

"What is happening to me? My throat aches. I read that there is a flu epidemic in the Netherlands." (Twitter)

\section{Information about the virus, prevention and treatment}

On some social networking sites, information about the virus was concerned with the difference between stomach flu and the seasonal flu, as well as the fact that flu is caused by a virus instead of being a bacterial infection.

"Stomach flu, right? A flu shot won't help with that because it's not really the flu." (Twitter)

Regarding prevention of the flu, there were several posts containing advice like going outside enough, getting enough rest, eating fruit and taking vitamins. Some Tweets suggested that vitamin $D$ and $C$ are effective in preventing the flu.

"Additional vitamin C! Vitamin C is a real virus killer. It has to be a high dose, a minimum of $3000 \mathrm{mg}$ per day. Can be more during flu." (Twitter)

There were several posts with links to news sites with prevention tips and information about the flu. Many posts on social networking sites were about the flu shot, of which several expressed belief in the effectiveness of the flu shot in preventing influenza.

"Luckily I got vaccinated and the variant is included (in the flu shot). Luckily, I won't get the flu!" (Twitter)

People either informed other people about their own positive experience with the flu shot, or reported that the flu shot had at least resulted in weaker flu symptoms.

"No, it's fine. I think $X$ had a small bout of flu, but thanks to the flu shot it was very weak." (Twitter)

Several people stated that they were planning to get the flu shot next time in order to prevent illness, which they were currently experiencing.

"I will see whether I can get the flu shot next year!! Again affected by one or another virus." (Twitter) 


\section{Uncertainty and mistrust}

There were also several posts expressing uncertainty regarding the flu shot. People who did not take the flu shot themselves wondered whether they should have taken it.

"The doctor also says that I should get the flu shot, but is it true? I strongly doubt it." (Twitter)

The majority of posts about the flu shot expressed doubt over its effectiveness in preventing the flu, and thought that it might even cause flu.

"Since I had the flu shot in November, I've had a double pneumonia and a strong cold. Went through the pain of the flu shot for nothing." (Twitter)

Others copied a link to a study that concluded that there is no evidence for the effectiveness of influenza vaccination.

"Summarized: 5700 studies about influenza vaccination, 31 were found to be done well and no clear evidence that influenza vaccination makes sense." (Twitter)

Several people stated that they did not believe in the effectiveness of the flu shot and advised others to not get vaccinated.

"Getting injected with diseases artificially, never start with that. Such a shot makes you ill in order to build up antibodies, but if you have the flu rarely you shouldn't get such a shot." (Facebook)

"Flu vaccination? Don't do it!!!" (Twitter)

Advice to not get vaccinated also seemed to influence uncertainty regarding influenza:

"Getting the flu shot this year after everything I've heard from others about their experiences?" (Twitter)

\section{Social media websites - March 2012}

\section{(Upcoming) epidemic}

In March 2012, there were some posts about the late flu epidemic on Twitter and Facebook. Most posts included links to public health websites or news sites. Some posts were written by representatives of the National Institute for Public Health and the Environment (RIVM). 
"X@RIVM: two things stand out about the influenza epidemic: it is mainly influenza A H3N2 and it has not been this late in 25 years." (Twitter)

Several posts, including news site links, indicated that the epidemic was expected to start soon in the Netherlands.

"There is a good chance that a national flu epidemic will strike this week. The north of the Netherlands was almost flu-free the past weeks, however with the end of the Easter vacation in this area, chances of infection will now increase." (Facebook)

In the beginning of March, there were several posts about the presence of an influenza epidemic, mostly including links to news sites. Furthermore, some people who posted about feeling ill received reactions that there was an epidemic in the Netherlands.

$X:$ "I am feeling ill. Lying in my bed since 9 o'clock, then cold, then again really hot... and I'm complaining. :-p \#stomach ache \#headache \#sigh" - Y: "Influenza virus is going around. Get well soon!" (Twitter)

\section{Information about influenza, prevention and treatment}

In March, several posts on Twitter referred to a website with statistics saying that approximately $40 \%$ of all Dutch people have the flu each year.

"Flu epidemic close: annually, approximately four out of ten Dutch people get the flu (link to website with statistics)." (Twitter)

Some social media posts informed the reader about the finding that there was less flurelated absenteeism from work, possibly because of the economic crisis.

"Less flu-related absenteeism because of the crisis (link to news site)." (Twitter)

At the same time, a number of Twitter users stated that news reports about the flu epidemic would give employees a reason for absenteeism.

"Employers watch out, the newspapers think it is necessary to give everyone a reason to stay at home. There seems to be a flu epidemic." (Twitter)

Readers were also presented with links to reports about hospitals, which were said to have had a lot of extra work during the epidemic. Several posts indicated a possible connection between metabolism of vitamin $\mathrm{D}$ and the flu.

$X:$ "There is no causal evidence for influenza virus -> flu. Vitamin D metabolism is [...] a far more logical explanation." - Y: "Vitamin D and influenza - 
Wikipedia (link) summary: influenza virus is bullshit, flu is a seasonal illness caused by a lack of Vitamin D." (Twitter)

In addition, people wondered why others worry about the on-going epidemic.

$X:$ "Why the fuck should you be worried about the flu epidemic? It is the FLU! Get over yourself!" - Y: "Sure, in the past 100 years only more than 20 million people died because of it. No big deal." - X: "That's what I mean. The flu you are suffering from during an epidemic is the same flu you would get without an epidemic." (Twitter)

Regarding flu prevention, a number of posts offered links to websites and summarized corresponding tips on how to stay healthy, including eating healthily, exposure to the sun, and wearing functional sportswear. Furthermore, vitamin D as a means of preventing flu infection was discussed again.

"The flu can be shortened or prevented with a high dose of vitamin D, but this is not possible with the flu shot." (Twitter)

Other posts expressing uncertainty were concerned with the question of whether "a mild epidemic" means that the epidemic is mild or that the flu is mild.

"You are wondering 'there is a mild flu epidemic in the Netherlands'... is it the flu that is mild or is it the epidemic?!" (Twitter)

Twitter users also asked whether the symptoms they were experiencing were normal for flu.

"I'm worried: does the recent flu have side-effects such as dizziness and prickling in hands and feet? I've had it for a week already..." (Twitter)

Some people stated that they were avoiding the flu shot because of its possible long term consequences.

As in February 2012, several posts on Twitter expressed belief in the effectiveness of the flu shot in preventing influenza. Several people stated that they had had good experiences with the flu shot and advised others to get the flu shot as well.

"What about the flu shot? Did you think about that yet? It works really well for me. Since taking the shot l've had no cold and wasn't ill anymore. \#tip" (Twitter)

Others stated that they were planning to get the flu shot next time.

"I think I will get a flu shot next year. (III again)" (Twitter) 
People belonging to the risk groups also posted about their annual flu shot.

\section{Uncertainty \& mistrust}

In March, uncertainty that was expressed on social media websites dealt with several different topics regarding influenza vaccination. Again, several people expressed uncertainty about the possibility of getting the flu in spite of being vaccinated.

"Oh yes, does a flu shot mean that you indeed can't get the flu anymore? That is what I'm wondering about." (Twitter)

Additionally, it was questioned whether it is a good idea to get vaccinated against influenza and if there are economic reasons for being invited to get the flu shot.

"Already flu complaints since 3 weeks; does the flu shot help or is it just an auxiliary income for general practitioners?" (Twitter)

Again, the majority of posts about the flu shot dealt with the ineffectiveness of it in terms of preventing the flu. People posted about their own or about others' experiences in this regard.

"I only know people who got really ill because of or despite of the flu shot." (Twitter)

Several posts discussed the harmful effects of the flu shot, including links about the flu shot causing Alzheimers and narcolepsy. Narcolepsy was thought to be caused by the pandemic (H1N1) flu shot from 2009.

"Mysterious sleep disease affects 50.000 Germans! Link to flu shot suggested in Finland. (link to website)." (Twitter)

Another related link was introduced with "All the reasons why you should not get the flu shot this year" on Twitter. Some people expressed that it was more or less a matter of luck whether one is protected by the flu shot or not.

$X:$ "Why do I always get the flu that isn't included in the flu shot????" - Y:

"Because the flu shot only protects you against half of the flu variants." (Twitter)

Some posts again indicated that there are economic reasons involved in the vaccination recommendations, which made them question the necessity of vaccination.

"It is getting more obvious that politics regarding vaccination are influenced by the principle 'the one who pays, decides.' (link) \#flu shot" (Twitter) 
Some posts discussed the collaboration between science and the pharmaceutical industry and questioned the reasons for this collaboration.

"The flu is a threat to our health, therefore, every year the flu shot has to be taken. Result: extra millions for the pharmaceutical industry and the state's finances! Explain to me why we Dutch people have to save millions?????????????????" (Facebook)

\section{Social media websites - April 2012}

\section{(End of) epidemic}

In April 2012, several Twitter users reported either that they themselves felt ill or that a lot of people around them were ill, and wondered whether a flu epidemic was occurring.

"I have the flu...I guess I'm in good company \#epidemic? \#at home" (Twitter)

Several posts informed readers that the flu epidemic of 2012 was over again and that it was comparably short.

"Flu News: Flu epidemic finished earlier than in other years: 'The flu epidemic had a rather short duration.' (link to news site)" (Twitter)

\section{Information about the virus, prevention and treatment}

Several times, links to websites providing statistics with regard to the flu were posted with information about the number of deaths.

"Two thousand additional deaths during the cold wave in February and the flu in March. (link to website with statistics)" (Twitter)

Furthermore, it was suggested that absenteeism should decrease again as a result of the end of the epidemic. In a number of Twitter-conversations, people advised each other to take time to get better after a flu infection.

$X:$ "Working through it is unreasonable, get well first." - Y: "You are right $X$; the flu will always disturb it; better to get additional rest and then hopefully get back into shape afterwards." (Twitter)

In April, several Twitter posts again expressed the belief that the flu shot is effective in preventing an infection or that it will at least weaken the symptoms of the flu. Furthermore, people informed others via Twitter that they were planning to get the flu shot next time. 


\section{Chapter 2}

"Maybe I have to get the flu shot every year." (Twitter)

\section{Uncertainty \& mistrust}

In April 2012, there were again also posts that expressed uncertainty about the possibility of getting the flu despite having had the flu shot.

"Really, are that many people ill?? I actually can't be ill because of the flu shot, but I am ill anyway, weird." (Twitter)

Most people that posted something about the flu shot reported being ill because of or despite of the flu shot.

"For the first time in my life I got a flu shot! For the first time in years I have the FLU!!!" (Twitter)

In addition, several posts speculated as to whether the flu shot is effective, or could actually be harmful to one's health.

"In this article, AGAIN the proof of the fact that vaccines don't work and are not well studied before they are given to thousands of people." (Facebook)

Furthermore, one link was posted a number of times that cites a Danish scientist, concluding that employers waste money with paying for the flu shot for their employees.

"Employers could better spend their money on improving hygiene in the workplace instead of on flu shots for their employees. (link to website)" (Twitter)

Moreover, a discussion on social media sites was visible about a news report that an employee of the National Vaccine Institute in 2009 had forgotten to put pandemic (H1N1) vaccines back in the refrigerator. As a result, 1.2 million vaccines were rendered useless.

"We never should have bought them in the first place. Fear. 'Millions of flu vaccines made useless through blunder' (link to news site)." (Twitter)

Views were expressed that a ban on any pay rise for two years was not an appropriate punishment for the mistake made by the employee concerned. Some posts provided links to news sites reporting that the RIVM and the minister of health of the Netherlands did not agree with the information that was given in the original press report. It was said to be exaggerated.

"RIVM: False article about destruction of flu vaccines in "national newspaper" (link to website)" (Twitter) 


\section{DISCUSSION}

The present study aimed to describe the online content of news sites and social media websites across a period of three months in which an influenza epidemic occurred in the Netherlands. By means of an online media monitoring program, more than 10.000 Dutch websites, including social media were scanned for the search terms influenza, vaccination, vaccine and epidemic during February, March and April 2012. Three different overarching themes were consistently identified: (1) the influenza epidemic, (2) more general information about the virus, prevention and treatment, and (3) uncertainty and mistrust regarding influenza vaccination.

With regard to content, news sites mainly reported about the progress of the influenza epidemic, the criteria for detecting an epidemic, and the type of virus that was identified in 2012. Most of the described content was neutral and informative and matched across different news sites. In line with our findings, Wright and Hinson (2009) found traditional news media to be more accurate, credible, ethical and truthtelling more often than social media. On social media websites, there were links to the same news sites, also reporting that a flu epidemic was approaching or already happening in the Netherlands. The difference was that on social media websites, the information that an epidemic was occurring was reported earlier than on news sites. This was mostly supported by user perceptions. At the same time, when news sites already reported the end of the epidemic, there were still posts on Twitter from users wondering whether there was a flu epidemic. This indicates that some people, instead of searching for news on the Internet, preferred to ask about this information on social media websites. This is in accordance with a notion outlined by Qualman (2011), who suggested that Internet users often trust the opinion of their peers more than that officials. Furthermore, the earlier appearance of information about influenza activity on social media websites is in accordance with research stating that certain information is registered earlier via social media sites than by official registration attempts (Asur \& Huberman, 2010; Marquet et al., 2006).

More general information about the influenza virus was also provided on news sites. This included explanations about differences between influenza and a common cold, symptoms of the flu, its seriousness, and possible complications, especially for young children and the elderly. Readers were further informed that the flu shot is the most effective means of prevention, but that there are also homeopathic remedies and that good physical health is important in preventing an infection. Moreover, readers could see that people are less likely to stay at home when they have been ill in the past years, possibly because of the economic crisis. Social media websites offered similar information about different kinds of infections. However, while these were stated as facts in news media, in social media this kind of information was subject to discussion. Additionally, advice for prevention of the flu was given, mostly supported by links to websites that offer advice. In contrast to what appeared on news sites, on 
social media sites vitamin $D$ and $C$ were often named as important for physical resistance to infections.

In the last category, uncertainty and mistrust, news sites and social media content both dealt with the debate about the collaboration between science, politics and the pharmaceutical industry. A topic that was discussed on more unconventional news sites was the possible dangerous consequences of flu vaccination, including conspiracy theories about controlling population growth and illnesses that are said to be caused by vaccines. On social media websites, flu was often said not to be a serious disease and there was a considerable amount of criticism regarding the flu shot. It was claimed that the flu is often taken as an excuse to not work. The belief that the national recommendations to get vaccinated were mostly driven by economic reasons was also expressed several times.

On social media websites, links to news media are posted and contents of those news sites are discussed. Additionally, as suggested by Asur and Huberman (2010), it is noticeable that several news sites, as well as public health websites, use social media to promote and spread information. It can be seen that especially on Twitter, links to news sites are shared and then re-shared by other people. As a result, information spreads quickly on a "many-to-many global platform" that is also used as a tool by a number of news sites and public health websites (Qualman, 2011).

One difference especially visible between news sites and social media websites was that the majority of news sites reported that influenza vaccination is the most effective or only effective means of preventing influenza infections. Whereas on social media websites, the majority of messages concerning the flu shot expressed views that the flu shot is not effective and may even be dangerous to one's health. Furthermore, whereas messages on social media websites that report that the flu shot is effective are mostly reports of one's own experience with the shot, anti-vaccination messages are often supported with links to supposedly scientifically proven articles from less objective websites. This is of concern, as it is conceivable that people who already distrust influenza vaccination will possibly feel their views to be confirmed by what they read and people who are unsure about the flu shot may be persuaded by what they are told is scientific evidence about vaccination. Moreover, across the three months that we monitored social networking sites, anti-vaccination messages were put online twice as much as pro-vaccination messages. Messages expressing uncertainty about influenza vaccination were present just as much as pro-vaccination messages. News media, on the other hand, presented information mainly in favor of influenza vaccination.

This study has been executed by means of a qualitative method. Therefore, the findings are descriptive in nature and do not enable us to make causal inferences about them, nor indicate relative importance of the different themes that emerged. Data collection and analysis was performed by only the first author. Not including a second coder could have biased the results and made it impossible to apply inter-rater 
reliability. It must be noted further that the distinction between the two Internet sources we described was based on the categorization that is made within the online media monitoring program Clipit. As a result, news websites included a wide range of different websites that generate original reports. These include print media that is additionally represented online, public health websites, but also more unconventional websites for people holding specific opinions. We decided not to further distinguish between those sources, because we think it is reasonable to believe that e-health users are directed to news about influenza and influenza vaccination in this broad sense or decide to engage in these topics on the social networking site of their choice. In addition, we were not able to obtain information on the number of followers and the characteristics of the readership. This information could have been helpful in stratifying the findings further in terms of importance and target group relevance.

\section{CONCLUSIONS}

News media and social media show some important similarities, as well as differences. The overarching themes identified on news sites and social media websites are roughly the same. However, particular topics seem to appear earlier on social networking sites, such as the occurrence of an influenza epidemic. Influenza is evaluated differently by the two media sources. In social media, influenza is often said not to be a serious disease. With regard to influenza vaccination, it is noticeable that there is considerably more criticism expressed on social media websites than on news sites. However, there are also a number of news sites that contradict Health Authority beliefs about the necessity of influenza vaccination. This might influence the success of vaccination campaigns and Health Authority vaccination recommendations. This study is a first step in identifying the importance of e-health in the formation of an opinion with regard to influenza vaccination. Future research should explore the specific impact of online media on decision making and actual behavior with regard to health information. 



\section{CHAPTER 3}

\section{Medical students' attitude towards influenza vaccination}

Published as:

Lehmann, B. A., Ruiter, R. A. C., Wicker, S., Chapman, G., \& Kok, G. (2015). Medical students' attitude towards influenza vaccination. BMC Infectious Diseases, 15, 185. 


\section{Chapter 3}

\section{ABSTRACT}

Influenza vaccination is recommended for all health care workers (HCWs) and most institutions offer vaccination for free and on site. However, medical students do not always have such easy access, and the predictors that might guide the motivation of medical students to get vaccinated are largely unknown. We conducted a crosssectional survey study among pre-clinical medical students in a German University hospital to assess the social cognitive predictors of influenza vaccination, as well as reasons for refusal and acceptance of the vaccine. Findings show that pre-clinical medical students have comparable knowledge gaps and negative attitudes towards influenza vaccination that have previously been reported among HCWs. Lower injunctive norms and higher feelings of autonomy contribute to no intention to get vaccinated against influenza, while a positive instrumental attitude and higher feelings of autonomy contribute to a high intention to get vaccinated. The variables in the regression model explained $20 \%$ of the variance in intention to get vaccinated. The identified factors should be addressed early in medical education, and hospitals might benefit from a more inclusive vaccination program and accessibility of free vaccines for their medical students. 


\section{INTRODUCTION}

Annual influenza epidemics are a major public health problem causing severe morbidity and mortality, especially in high risk groups. High risk groups include children younger than 2, people over the age of 65, and patients with medical conditions that make them more likely to suffer from influenza-related complications (Mertz et al., 2013; WHO, 2005). Health care workers (HCWs) can serve as vectors in the transmission of influenza to vulnerable patients and are therefore recommended to get vaccinated against influenza annually (Amodio et al., 2014; Bénet et al., 2012; CDC, 2011; WHO, 2009). Most hospitals and medical institutions offer their HCWs vaccination for free and on site. In contrast, such easy access to the vaccine is not always offered to their medical students (Talbot, Dellit, Hebden, Sama, \& Cuny, 2010), even though they have regular patient contact throughout their education.

Research has repeatedly shown that a large proportion of HCWs have unfavorable attitudes towards influenza vaccination, and the reasons for rejecting or accepting influenza vaccination have been examined extensively (Aguilar-Díaz, Jiménez-Corona, \& Ponce-de-León-Rosales, 2011; Christini, Schutt, \& Byers, 2007; Cohen \& Casken, 2012; Hofmann, Ferracin, Marsh, \& Dumas, 2006; Hollmeyer, Hayden, Poland, \& Buchholz, 2009; Looijmans-van den Akker et al., 2009; Martinello, Jones, \& Topal, 2003; Talbot et al., 2010; Van den Dool et al., 2008; Wicker, Rabenau, Doerr, \& Allwinn, 2009). However, only a few studies included medical students (Christini et al., 2007; Martinello et al., 2003; Talbot et al., 2010). Finding out about the factors that predict medical students' motivation to get vaccinated against influenza can have important implications for the education of these students in terms of developing a favorable attitude towards influenza vaccination and addressing knowledge gaps. Moreover, it can have implications for hospitals in terms of motivating their students to get vaccinated against influenza annually and to include them in vaccination programs. After all, medical students have patient contact throughout their education, and they are the physicians of the future. Therefore, we conducted a cross-sectional survey to investigate the social cognitive variables that predict the influenza vaccination intention of medical students.

The few existing studies that included medical students (Christini et al., 2007; Martinello et al., 2003; Talbot et al., 2010), exclusively investigated post-hoc reasons for acceptance and refusal of the vaccine, while the predictors that might guide the motivation of medical students are largely unknown. In the current study, we therefore utilized measures of constructs from the Reasoned Action Approach (RAA) in our cross-sectional survey (Fishbein \& Ajzen, 2010). The RAA is a social cognition model of human behavior, which proposes that the motivation or intention to perform a behavior is caused by attitudes, perceived norms, and perceived behavioral control. Attitudes are a person's overall evaluation of the anticipated advantages and disadvantages resulting from performing a behavior. Perceived norms refer to the anticipat- 
ed approval or disapproval of significant others concerning performance of a health behavior and also whether comparable others perform the behavior themselves. Finally, perceived behavioral control refers to the belief regarding degree of perceived capacity and autonomy in performing the behavior. The RAA has been successfully used to predict the influenza vaccination intentions and behavior of HCWs (Lehmann, Ruiter, Chapman, \& Kok, 2014). Attitude has been shown to be one of the strongest predictors of the intention to get vaccinated. Medical students who are lower in hospital hierarchies might be more susceptible to perceived norms than other HCW groups.

This study investigated the social cognitive variables that predict medical students' intention, as well as reasons for refusal and acceptance to get vaccinated against influenza. Results of this cross-sectional study can assist in the development of future educational programs for medical students and can provide advice to hospitals about how to include medical students into their annual HCW vaccination programs.

\section{METHODS}

\section{Participants and procedure}

Pre-clinical medical students at the University Hospital Frankfurt have to attend an occupational health screening before their preliminary medical examination, at the end of their second year. In May 2012 and 2013, these students were asked to fill in a cross-sectional survey about the factors influencing the decision to get vaccinated against influenza ( $N=264$ in 2012, $N=279$ in 2013).

\section{The questionnaire}

The first questionnaire conducted in 2012 consisted of 18 questions, answered on 7point Likert scales, unless otherwise indicated. Demographic measures included age, sex and current vaccination status. Table 3.1 provides an overview of the questions about social cognitive variables utilized in the survey.

Additionally, all participants were asked to indicate which of a set of 8 facilitating factors of influenza vaccination would apply to them (self-protection, patient protection, protection of family and friends, work ethic to not infect anyone, advice from a medical expert, to set a positive example for patients, vaccination available for free, vaccination is safe) and exclusively non-immunizers were asked to indicate which of a set of 9 inhibiting factors apply to their decision to not get vaccinated (no specific risk, influenza is not a serious disease, fear of side-effects, vaccination provides insufficient protection, influenza vaccination was never offered to me, vaccination could cause flu, no possibility to get vaccinated, medical contraindication, fear of needles). Multiple answers were possible. 
Table 3.1 Overview of constructs measured by the survey

\begin{tabular}{|c|c|}
\hline Variable & Questions \\
\hline Instrumental attitude & $\begin{array}{l}\text { Getting vaccinated against influenza every year in October/ November would } \\
\text { be: very good - very bad }\end{array}$ \\
\hline Experiential attitude & $\begin{array}{l}\text { When I think of getting vaccinated against influenza annually, it makes me: very } \\
\text { anxious - not at all anxious }\end{array}$ \\
\hline Injunctive norm & $\begin{array}{l}\text { Most people who are important to me think I should get vaccinated against } \\
\text { influenza annually. agree - disagree }\end{array}$ \\
\hline Descriptive norm & $\begin{array}{l}\text { Most physicians get vaccinated against influenza annually. very unlikely - very } \\
\text { likely }\end{array}$ \\
\hline Capacity & $\begin{array}{l}\text { I am confident that I can get vaccinated against influenza next October/ } \\
\text { November, if I want to. true - false. }\end{array}$ \\
\hline Autonomy & Getting vaccinated against influenza annually is up to me. agree - disagree \\
\hline $\begin{array}{l}\text { Behavioral beliefs (self- } \\
\text { protection and patient } \\
\text { protection) }\end{array}$ & $\begin{array}{l}\text { Getting vaccinated against influenza annually will result in fewer influenza } \\
\text { infections and less work-absenteeism. very likely - very unlikely; } \\
\text { Getting vaccinated against influenza annually will prevent at-risk patients from } \\
\text { getting influenza. true - false }\end{array}$ \\
\hline $\begin{array}{l}\text { Knowledge about } \\
\text { recommendations }\end{array}$ & $\begin{array}{l}\text { I know about the national recommendations for health care workers to get } \\
\text { vaccinated against influenza annually, in order to protect themselves and } \\
\text { patients against influenza infections. true - false }\end{array}$ \\
\hline Injunctive normative belief & $\begin{array}{l}\text { My future employer will think that: I should - I should not get vaccinated } \\
\text { against influenza annually. }\end{array}$ \\
\hline Control belief & $\begin{array}{l}\text { I expect that most hospitals enable their employees to get vaccinated against } \\
\text { influenza annually at work. very likely - very unlikely. }\end{array}$ \\
\hline Intention & $\begin{array}{l}\text { I intend to get vaccinated against influenza next October/ November. very } \\
\text { unlikely - very likely. }\end{array}$ \\
\hline Barrier (high workload) & $\begin{array}{l}\text { Imagine that on the day you get offered influenza vaccination at work, you are } \\
\text { under a lot of time pressure and barely have time to take a break. How likely is } \\
\text { it that you will still get vaccinated against influenza? very likely - very unlikely }\end{array}$ \\
\hline
\end{tabular}

Some of the questions used for analyses came from an attached questionnaire utilized by one of the co-authors of this study. Due to an unforeseen change in the set-up of the study, these questions were missing for the 2013 sample. The questionnaire conducted in 2013 was missing the demographic questions and the questions about facilitating and inhibiting factors of influenza vaccination, resulting in 14 questions from the previous questionnaire.

\section{Data analysis}

SPSS 20.0 was used to analyze the data. Following a descriptive analysis of the sample (frequencies), univariate associations between intention and social cognitive variables were analyzed with Pearson correlation coefficients. Significance was set at $p \leq .05$. Intention was not normally distributed, and thus we classified responses into three groups; no intention to get vaccinated against influenza $(0=1.0-2.0)$, not having made a clear decision about vaccination $(1=2.5-5.5)$, and a high intention to get vaccinated 
$(2=6.0-7.0)$. Therefore, multinominal logistic regression was used to examine the effects of the independent variables on the probability of (1) having no intention to get vaccinated vs. not having made a clear decision and (2) having a high intention to get vaccinated vs. not having made a clear decision. We checked for multicollinearity by inspecting the VIF and Tolerance values.

\section{RESULTS}

\section{Descriptive statistics}

The 2012 sample consisted of 264 pre-clinical medical students (see Table 3.2), with 91 males (34.5\%) and 173 females (65.5\%) and a mean age of 23 years (range 20 to 47). Of these students, 34 (12.9\%) were vaccinated against influenza. The 2013 sample consisted of 279 German medical students. In 2012, 107 (41\%) of the participants reported having no intention to get vaccinated against influenza, 99 (37\%) indicated not having made a clear decision, and $57(22 \%)$ reported a high intention to get vaccinated. In 2013, these numbers were highly similar with 115 (41\%), 100 (36\%), and 63 (23\%) respectively.

Table 3.2 Students' demographics and vaccination characteristics (2012 and 2013)

\begin{tabular}{|c|c|c|c|}
\hline & $\begin{array}{l}2012 \\
(N=264,48.6 \%)\end{array}$ & $\begin{array}{l}2013 \\
(N=279,51.4 \%)\end{array}$ & $\begin{array}{l}\text { Total } \\
(\mathrm{N}=543)\end{array}$ \\
\hline Male & $91(34.5)$ & N.A. & N.A. \\
\hline Female & $173(65.5)$ & N.A. & N.A. \\
\hline Mean age (S.D.) & $23.1(3.32)$ & N.A. & N.A. \\
\hline Vaccinated & 34 (12.9) & N.A. & N.A. \\
\hline No intention & $107(41)$ & $115(41)$ & $222(41)$ \\
\hline No clear decision & $99(37)$ & $100(36)$ & $199(36.7)$ \\
\hline High intention & $57(22)$ & $63(23)$ & $120(22.1)$ \\
\hline
\end{tabular}

Data are reported as number of participants (\%).

Data not available (N.A.).

Table 3.3 shows the facilitating and inhibiting factors that participants of the 2012 sample were asked to choose from as reasons to accept or reject influenza vaccination. Among the facilitating factors, self-protection, patient protection, and the protection of family and friends were the most frequently chosen reasons in favor of influenza vaccination by both non-immunizers and immunizers. Logistic regression analysis revealed that the facilitating factors account for $25 \%$ of the explained variance in vaccination uptake (Nagelkerke $R^{2}=.25$ ). Among the inhibiting factors, not being at a specific risk was chosen most often by non-immunizers ( $N=116,50.4 \%)$, followed by 
thinking that influenza is not a serious disease $(N=51,21.7 \%)$. Other relatively common reasons were fear of side-effects $(N=46,20 \%)$, thinking that vaccination provides insufficient protection $(N=44,19.1 \%)$, and that influenza vaccination had never been offered $(N=39,17 \%)$. Less common reasons were the belief that the vaccination could cause flu $(N=13,5.7 \%)$, not having had the possibility to get the vaccination $(N=6$, $2.6 \%)$, a medical contraindication $(N=5,2.2 \%)$, and fear of needles $(N=2,0.9 \%)$.

Table 3.3 Facilitators and inhibitors of influenza vaccination (2012 sample)

\begin{tabular}{|c|c|c|c|c|c|}
\hline Factors & $\begin{array}{l}\text { Not Vaccinated } \\
(\mathrm{N}=\mathbf{2 3 0}, \mathbf{8 7 \%})\end{array}$ & $\begin{array}{l}\text { Vaccinated } \\
(\mathrm{N}=34,3 \%)\end{array}$ & Total $(\mathrm{N}=\mathbf{2 6 4})$ & OR $(95 \% \mathrm{Cl})$ & p-value \\
\hline \multicolumn{6}{|l|}{ Facilitators } \\
\hline Self-protection & $154(67)$ & $32(94.1)$ & $186(70.5)$ & $9.62(2.12-43.55)$ & .003 \\
\hline Patient protection & 140 (60.9) & $30(88.2)$ & $170(64.4)$ & $6.08(1.83-20.13)$ & .003 \\
\hline Family and friends & $106(46.1)$ & $12(35.3)$ & $118(44.7)$ & $0.18(0.07-0.46)$ & $<.001$ \\
\hline Work ethics & $65(28.3)$ & $14(41.2)$ & 79 (29.9) & $1.53(0.59-3.94)$ & .38 \\
\hline Medical advice & $42(18.3)$ & $4(11.8)$ & $46(17.4)$ & $0.57(0.16-2.09)$ & .40 \\
\hline Set positive example & $36(15.7)$ & $6(17.6)$ & $42(15.9)$ & $0.87(0.25-2.95)$ & .82 \\
\hline Free of charge & $34(14.8)$ & $4(11.8)$ & $38(14.4)$ & $0.72(0.19-2.73)$ & .63 \\
\hline Flu shot is safe & $32(13.9)$ & $7(20.6)$ & $39(14.8)$ & $2.78(0.79-9.75)$ & .11 \\
\hline \multicolumn{6}{|l|}{ Inhibitors } \\
\hline No specific risk & $116(50.4)$ & - & $116(43.9)$ & & \\
\hline No serious disease & $51(21.7)$ & - & $51(19.3)$ & & \\
\hline Fear of side-effects & $46(20)$ & - & $46(17.4)$ & & \\
\hline Insufficient protection & $44(19.1)$ & - & $44(16.7)$ & & \\
\hline Never offered & $39(17)$ & - & $39(14.8)$ & & \\
\hline Causes flu & $13(5.7)$ & - & $13(4.9)$ & & \\
\hline No possibility & $6(2.6)$ & - & $6(2.3)$ & & \\
\hline Medical contraindication & $5(2.2)$ & - & $5(1.9)$ & & \\
\hline Fear of needles & $2(0.9)$ & - & $2(0.8)$ & & \\
\hline
\end{tabular}

Data are reported as number of participants (\%). Note $R^{2}=.25$

\section{Correlates of intention to get vaccinated}

Table 3.4 shows the correlates of intention to get vaccinated of students in 2012 in the bottom half and students in 2013 in the upper half. A small effect is $r=.10-.23$, a moderate effect $r=.24-.36$ and a large effect is $r \geq .37$ (Cohen, Cohen, West, \& Aiken, 2003). 


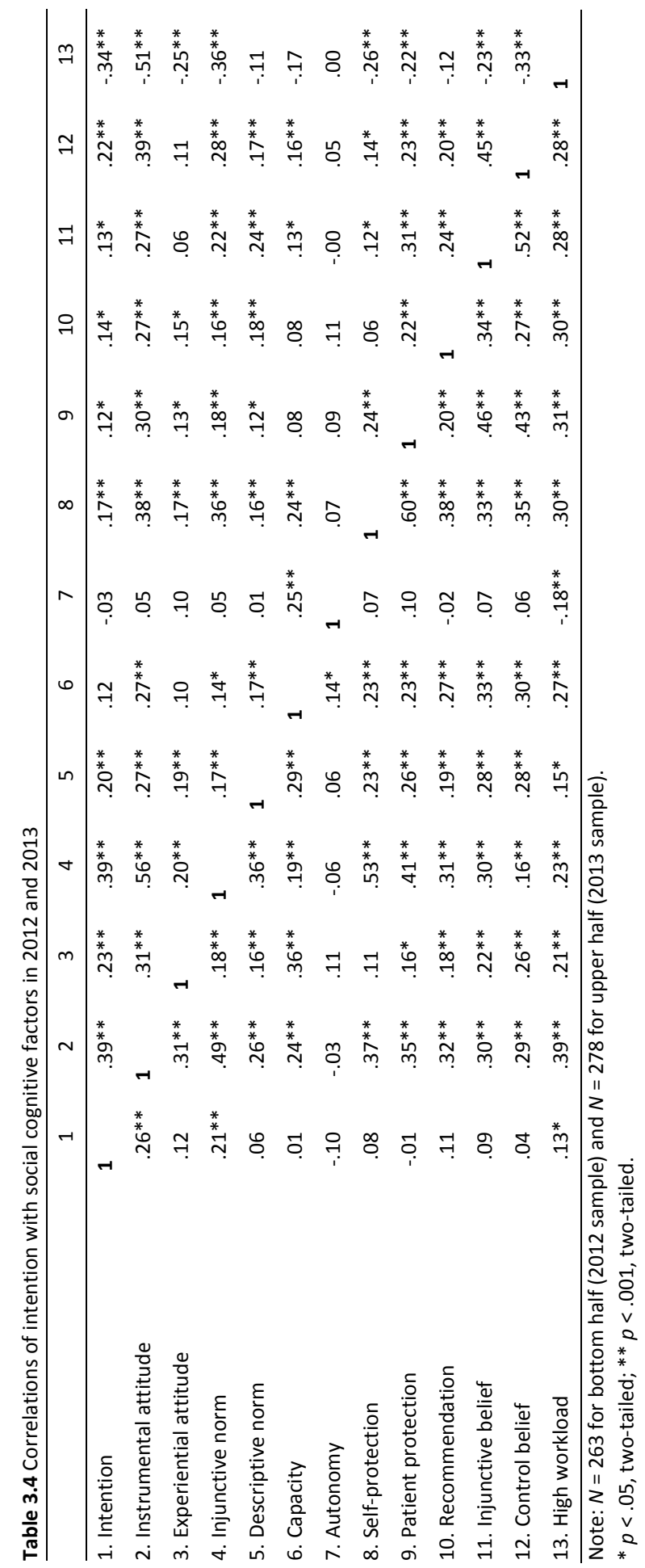


In 2012, we found a moderate positive univariate association with intention for instrumental attitude, and small positive associations with injunctive norm and high workload. In 2013, strong positive associations were found for instrumental attitude and injunctive norm. A moderate negative association was found for the barrier of high workload. Experiential attitudes, descriptive norm, self-protection, patient protection, knowledge about national recommendations, injunctive belief, and control belief all showed a small univariate association with intention in 2013 (see Table 3.4).

\section{Multinominal logistic regression}

In the influenza season $2012 / 13$, some influenza vaccines of the provider Novartis had to be retracted temporarily in some European countries, including Germany. Flocculation had been observed in some vaccines, which is the formation of visible clusters due to clumps of protein particles, and it had been recalled by the provider as a safety measure. This could have potentially affected the comparability of student responses in 2013 and we formally tested for differences between both samples because of this incident by examining possible interaction effects of the predictor variables with sample (2012 vs. 2013). Except for two interaction terms (high workload for no intention vs. no clear decision; descriptive norm for high intention vs. no clear decision), no significant interaction terms were found, suggesting no systematic evidence for an impact of sample (i.e. Novartis incident) on the relationships of the predictor variables with intention. The interaction terms were therefore removed from the model after which the analyses were repeated. Results of the multinominal logistic regression across samples are shown in Table 3.5.

Medical students with lower injunctive norms and higher feelings of autonomy were significantly more likely to have no intention to get vaccinated vs. not having made a clear decision. Having a positive instrumental attitude and higher feelings of autonomy significantly increased the probability of having a high intention to get vaccinated vs. not having made a clear decision. The variables in the regression model explained $20 \%$ of the variance in intention (Nagelkerke Pseudo $R^{2}=.20$ ), with a classification accuracy of $55.2 \%$. There was no significant contribution of sample to the prediction of intention, indicating that there was no difference in intention between both samples after the Novartis incident took place. Inspection of the VIF and tolerance values did not indicate any cause for concern with regard to multicollinearity.

\section{Additional analyses}

In an exploratory manner we excluded the most influential variables autonomy and injunctive norm from the multinominal analysis and found that negative instrumental attitude became a significant predictor of no intention to get vaccinated as opposed to an unclear decision when injunctive norm was excluded. Therefore, we conducted a binary logistic regression using a bootstrapping technique (Preacher \& Hayes, 2004) to 


\section{Chapter 3}

analyze whether injunctive norm mediated the relationship between instrumental attitude and intention (no intention/ unsure; $N=420$ ).

Table 3.5 Multinominal logistic regression

\begin{tabular}{|c|c|c|c|c|c|}
\hline Predictors & $\mathbf{r}$ & b & S.E. & Wald & $\mathbf{p}$ \\
\hline \multicolumn{6}{|c|}{ No intention vs. no clear decision $(N=421)$} \\
\hline Instrumental attitude & $-.23 * *$ & -1.47 & .09 & 2.85 & .09 \\
\hline Experiential attitude & $-.10^{*}$ & -.10 & .07 & 2.01 & .16 \\
\hline Injunctive norm & $-.28 * *$ & -.24 & .07 & 11.23 & .001 \\
\hline Descriptive norm & -.04 & .06 & .08 & .62 & .43 \\
\hline Capacity & .01 & .03 & .05 & .33 & .57 \\
\hline Autonomy & $.20 * *$ & .33 & .09 & 13.30 & $<.001$ \\
\hline Self-protection & $-.10^{*}$ & .01 & .07 & .03 & .87 \\
\hline Patient protection & -.06 & .04 & .07 & .35 & .56 \\
\hline Recommendation & -.09 & -.04 & .07 & .36 & .55 \\
\hline Injunctive belief & $-.11 *$ & -.06 & .10 & .43 & .51 \\
\hline Control belief & $-.11 *$ & -.07 & .09 & .61 & .44 \\
\hline High workload & $.10^{*}$ & .09 & .05 & 2.68 & .10 \\
\hline Sample 2012 & .02 & .03 & .22 & .02 & .90 \\
\hline Sample 2013 & . & . & . & . & . \\
\hline \multicolumn{6}{|c|}{ High intention vs. no clear decision $(N=319)$} \\
\hline Instrumental attitude & $.20 * *$ & .29 & .11 & 7.28 & .01 \\
\hline Experiential attitude & $.14^{*}$ & .09 & .10 & .87 & .35 \\
\hline Injunctive norm & .10 & -.00 & .08 & .00 & .99 \\
\hline Descriptive norm & .11 & .09 & .09 & 1.12 & .29 \\
\hline Capacity & .08 & -.03 & .07 & .18 & .67 \\
\hline Autonomy & $.26 * *$ & .24 & .11 & 5.19 & .02 \\
\hline Self-protection & .04 & -.03 & .09 & .13 & .72 \\
\hline Patient protection & .02 & -.06 & .08 & .55 & .46 \\
\hline Recommendation & .07 & -.00 & .08 & .00 & .96 \\
\hline Injunctive belief & .02 & -.04 & .12 & .08 & .77 \\
\hline Control belief & .03 & -.05 & .11 & .20 & .65 \\
\hline High workload & -.03 & .01 & .06 & .01 & .91 \\
\hline Sample 2012 & .02 & -.08 & .25 & .11 & .74 \\
\hline Sample 2013 & . & . & . & . & . \\
\hline Pseudo $R^{2}$ & & .20 & & & \\
\hline Classification accuracy (\%) & & 55.2 & & & \\
\hline
\end{tabular}

$* p<.05$, two-tailed; ** $p<.01$, two-tailed. 
The bias corrected and accelerated ( $\mathrm{BCa}$ ) confidence intervals were set at 0.95 with 5000 resamples. In the mediation analysis, instrumental attitude was the independent variable, intention (no/ unsure) was the dependent variable, and injunctive norm was the mediator. Results revealed a significant mediation effect of injunctive norm on the relationship between instrumental attitude and intention $(b=.14, \mathrm{BCa} 95 \% \mathrm{Cl}[.074$; .225]; see Figure 3.1).

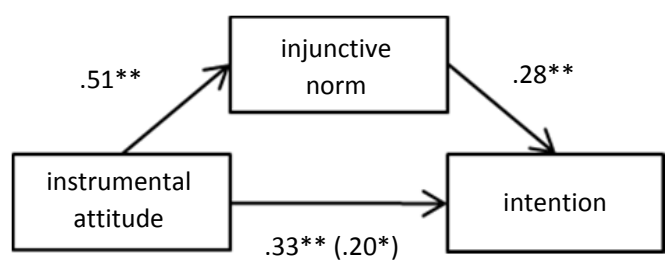

Figure 3.1 Regression coefficients for the relationship between instrumental attitude and intention to get vaccinated (no/ unsure) as mediated by injunctive norm. The path between instrumental attitude and injunctive norm is an OLS regression coefficient, while the other paths are logistic regression coefficients. The logistic regression coefficient between instrumental attitude and intention, controlling for injunctive norm, is in parentheses.

$* p<.05 ;{ }^{* *} p<.01$

\section{DISCUSSION}

This cross-sectional study aimed at identifying the social cognitive variables that predict influenza vaccination intentions of German medical students and their reasons for refusal and acceptance of vaccination. We identified only few studies that focused exclusively on medical students (Betsch \& Wicker, 2012; Hernández-García, 2012; Machowicz et al., 2010; Milunic, Quilty, Super, \& Noritz, 2010; Wicker et al., 2013). Two of these studies report insufficient knowledge (Betsch \& Wicker, 2012; Hernández-García, 2012), while the other three studies focus on medical students' reasons for acceptance and refusal of influenza vaccination (Machowicz et al., 2010; Milunic et al., 2010; Wicker et al., 2013). In these cross-sectional studies, reported vaccination coverage rates range from 4.7 to $58.1 \%$. In accordance, this study showed that only a small proportion of the medical students were motivated to get vaccinated against influenza in both samples ( $22 \%$ and $23 \%$ respectively) and that an even smaller proportion (12.9\%) had been vaccinated in 2012 . This is also consistent with the vaccination coverage rates of other HCW groups reported in European health care settings, including some of the studies about medical students (Betsch \& Wicker, 2012; Blank, Schwenkglenks, \& Szucs, 2009; Costantino et al., 2014; Endrich, Blank, \& Szucs, 2009; Hernández-García, 2012; Machowicz et al., 2010). However, some studies that includ- 
ed students had reported considerably higher vaccination rates (Christini et al., 2007; Martinello et al., 2003; Milunic et al., 2010). Some studies had additionally found that clinical medical students are more likely to be vaccinated than pre-clinical students (Milunic et al., 2010; Wicker et al., 2013), while another study had found no difference between these two groups of students (Hernández-García, 2012).

Reasons for accepting the vaccine found in previous studies were self-protection, patient protection, and that the vaccine was offered for free (Machowicz et al., 2010; Milunic et al., 2010; Wicker et al., 2013). One study additionally found professional ethics, setting an example for patients, vaccine safety and the recommendation for HCWs to get vaccinated as facilitating factors (Wicker et al., 2013). In the current study, the most common reasons reported for getting vaccinated against influenza were self-protection, patient protection and the protection of family and friends. Reasons for refusal of the vaccine reported in previous studies were inconvenience, forgetfulness, concerns about side-effects, perceiving vaccination as being unnecessary, and the cost of the vaccine. Students further indicated a low risk perception, laziness and lack of knowledge (Betsch \& Wicker, 2012; Hernández-García et al., 2012; Machowicz et al., 2010; Milunic et al., 2010; Wicker et al., 2013). Reported reasons for not getting vaccinated in the current study were mostly associated with a low risk perception, fear of side-effects, and the disbelief in the effectiveness of influenza vaccination. To a lesser extent, organizational barriers were revealed to be a possible inhibiting factor, mirroring factors associated with refusal of influenza vaccination in other studies (Christini et al., 2007; Costantino et al., 2014; Milunic et al., 2010). As was mentioned before, easy access to the vaccine is not always offered to medical students (Talbot et al., 2010), even when they have regular patient contact.

Results further suggested that participants who did not expect important others to want them to get vaccinated were more likely to have no intention to get vaccinated as opposed to being unsure about their future vaccination intentions. Injunctive norm additionally mediated the relationship between instrumental attitude and intention. This is surprising since in the RAA, perceived norms and attitude predict intention independently (Fishbein \& Ajzen, 2010). Our findings suggest that medical students that have a negative instrumental attitude towards influenza vaccination might be even more susceptible to negative injunctive norms that they might encounter when entering a clinic, and that these two determinants predict their intention to get vaccinated. One possible explanation for this is that medical students are much more susceptible to injunctive norms in general because of their lower status in the health care hierarchy, as opposed to other HCW groups. This stresses the importance of intervening early in medical students' education so that they form the right instrumental attitudes towards influenza vaccination before entering clinics. Moreover, higher feelings of autonomy in the decision whether to get vaccinated increased the probability of having no intention to get vaccinated as opposed to being unsure. A high intention to get vaccinated was most likely for participants who had a positive instrumental 
attitude and who also had high feelings of autonomy. Scores on autonomy were generally very high, suggesting that medical students feel completely free to choose whether to get vaccinated against influenza. High feelings of autonomy do not seem to be a barrier, as long as they are paired with positive instrumental attitudes.

The mentioned factors are significant but relatively weak predictors of the intention to get vaccinated against influenza. This indicates that there might be additional factors involved in the motivation of students to get vaccinated. The factors included in the multinominal logistic regression did not capture the organizational issues suggested in other studies, such as the inconvenience of getting vaccinated, not being offered vaccination, or getting vaccination for free (Christini et al., 2007; Milunic et al., 2010). However, previous studies have shown that these factors are perceived barriers to vaccination, and hospitals should therefore increase the accessibility of free vaccines to medical students and include them more actively in vaccination programs. This could also be an explanation for why the percentage of students who are vaccinated is smaller than the percentage of students who intend to get vaccinated against influenza. This intention-behavior gap has been identified across a broad range of health behaviors, including influenza vaccination (Lehmann et al., 2014; Sheeran, 2002).

This is one of the few studies to investigate the factors preceding the intention to get vaccinated among medical students. However, this study has some limitations worth mentioning. Firstly, the survey included only 18 items to reduce the length and to increase the response rate. Including more items that capture factors identified in other studies could have improved the predictive power of our model. Secondly, due to an unforeseen change in the set-up of the study, the second sample was missing questions on demographics, facilitating and inhibiting reasons, as well as the vaccination status of the participants. Therefore, we were only able to compare the intention to get vaccinated and its possible predictors across the two samples. We cannot say anything about differences in the number of people who were vaccinated. However, intention did not differ between the two years, making it likely that we would not have found considerable differences in vaccination rates between the two samples.

\section{CONCLUSIONS}

In conclusion, this study showed that pre-clinical medical students have comparable knowledge gaps and negative attitudes with regard to influenza vaccination that have been reported among HCWs already working in hospital settings. Education about influenza and vaccination should therefore be addressed early during medical training, and the importance of influenza vaccination should be taught so as to develop more favorable attitudes towards vaccination. 



\section{CHAPTER 4}

\section{"I don't see an added value for myself"}

Published as:

Lehmann, B. A., Ruiter, R. A. C., Wicker, S., van Dam, D., \& Kok, G. (2014). “I don't see an added value for myself": A qualitative study exploring the social cognitive variables associated with influenza vaccination of Belgian, Dutch and German healthcare personnel. BMC Public Health, 14, 407. 


\section{ABSTRACT}

Health Authorities recommend influenza vaccination of health care workers (HCWs) to decrease the transmission of influenza to vulnerable patients. Recent studies have almost exclusively used quantitative questionnaires in order to identify determinants of vaccination behavior. Interviews enable HCWs to express freely why they think they are (not) willing to get vaccinated against influenza. By means of semi-structured oneon-one interviews with 123 Belgian, Dutch and German HCWs, reasons for and against vaccination, experiences with influenza vaccination, intention to get vaccinated and possible barriers, as well as willingness to advice influenza vaccination to patients were investigated. Data were processed with QSR NVivo 8.0 and analyzed using a combination of a deductive and a general inductive approach. Across countries, self-protection, patient protection, and protection of family members were reported as most important reasons to get vaccinated against influenza. Reasons to not get vaccinated against influenza were fear of side effects caused by the vaccine, a low risk perception, the disbelief in the effectiveness of influenza vaccination, organizational barriers, misconceptions, and undefined negative emotions. The social cognitive variables underlying the decision of HCWs to get vaccinated against influenza (or not) seem to be similar in Belgium, Germany, and the Netherlands, even though some differences surfaced. A quantitative investigation of those social cognitive variables is needed in order to determine the importance of the social cognitive variables in explaining the intention to get vaccinated and the importance of the similarities and differences between countries that have been found in this study. 


\section{INTRODUCTION}

Annual influenza epidemics are a public health problem resulting in up to five million cases of severe illness worldwide, of which 5 to $10 \%$ result into deaths each year (WHO, 2009). Affected are especially children, the elderly and patient groups with specific health conditions (Mertz et al., 2013; WHO, 2005). Health Authorities recommend influenza vaccination of health care workers (HCWs) to decrease the transmission of influenza to vulnerable patients (WHO, 2009; CDC, 2011). Studies have shown that vaccination of HCWs can decrease clinical disease in healthy adults by $70-90 \%$ and might decrease all-cause mortality by $29 \%$ (Ahmed, Lindley, Allred, Weinbaum, \& Grohskopf, 2014; WHO, 2005). Moreover, less influenza infections among HCWs has, amongst other economic benefits, the advantage of less illness-related workabsenteeism within this group (Burls et al., 2006; Nichol et al., 1995).

Influenza vaccination has been shown to be safe and effective (Couto et al., 2012; Nichol et al., 1995) and can be given relatively effortless to a large group of people. Although benefits are clearly demonstrated (Burls et al., 2006; Nichol et al., 1995) and hospitals simplified the process of HCWs getting vaccinated by offering free vaccine on work-site and by giving necessary information (Doratotaj, Macknin, \& Worley, 2008; Llupià et al., 2010; McLennan \& Wicker, 2010), the actual vaccination numbers are generally low and stay far below Health Authority recommendations (Maltezou et al., 2008; Van Essen, Palache, Forleo, \& Fedson, 2003; WHO, 2005). A study comparing 11 European countries found vaccination coverage rates of 6.4 to $26.3 \%$ among HCWs (Blank, Schwenkglenks, \& Szucs, 2009).

Next to the professional responsibility of HCWs to get vaccinated against influenza in order to protect their patients (Talbot, 2014), HCWs and medical practitioners in particular are expected to advise influenza vaccination to their patients. However, HCWs do not always advise vaccination to their patients (Zimmerman et al., 2003).

Four reviews explored the social cognitive reasons reported by HCWs for (not) getting vaccinated against influenza (Aguilar-Díaz, Jiménez-Corona, \& Ponce-de-LeónRosales, 2011; Cohen \& Casken, 2012; Hofmann, Ferracin, Marsh, \& Dumas, 2006; Hollmeyer, Hayden, Poland, \& Buchholz, 2009). The most common reasons for rejection are a low risk perception, doubts about the effectiveness of vaccines, fear of sideeffects, and the belief that influenza is not a serious illness. The most common reasons in favor of influenza vaccination are self-protection and the belief in the effectiveness of the vaccine. Older age and previous receipt of influenza vaccination were additionally shown to predict the intention to get vaccinated (Aguilar-Díaz et al., 2011; Cohen \& Casken, 2012; Hofmann et al., 2006; Hollmeyer et al., 2009).

Recent studies have almost exclusively used quantitative questionnaires in order to identify determinants of vaccination behaviour (Aguilar-Díaz et al., 2011; Cohen \& Casken, 2012; Hofmann et al., 2006; Hollmeyer et al., 2009). In these questionnaires, $\mathrm{HCW}$ receive constructed reasons against and in favor of influenza vaccination and 
are forced to choose. This may lead HCWs to select answers even if they do not reflect their true reasons for (not) getting vaccinated. As was proposed by Fishbein and Ajzen (2010), social cognitive variables and their underlying behavioral, normative and control beliefs should be elicited by asking people directly about them. This gives HCWs the opportunity to express without restriction why they think they are (not) willing to get vaccinated against influenza. The study was conducted in three European countries, one of them not having been systematically surveyed before (Belgium). In this study, one-to-one interviews with HCWs of hospitals in Belgium, Germany and the Netherlands were used in order to gain a direct and more in-depth understanding of the beliefs underlying the decision to get vaccinated against influenza of HCWs that are already known, as well as allowing for the possibility to identify beliefs that have not been captured by previous quantitative studies.

\section{METHODS}

\section{Participants and procedure}

Three hospitals participated in this study: the University Hospital Antwerp, in Belgium, the University Hospital Frankfurt, in Germany, and the Orbis Medisch Centrum, in the Netherlands. These three hospitals were chosen, because of existing contacts to either the occupational physician or the clinical microbiologist, the existence of comparable vaccination programs, and having a substantial number of the HCWs not taking the vaccination (see Table 4.1 for distribution of immunizers and non-immunizers). All three hospitals have an annual vaccination program in which employees are encouraged to get vaccinated against influenza, they are informed about influenza vaccination and offered vaccination for free and during their working hours. However, these programs are not based on extensive social psychological investigations of the reasons HCWs have for getting vaccinated or not. Possible participants were approached during their lunch breaks or in the waiting room of the occupational physician. Participation was open to all HCWs. Particular effort was done to obtain a comparable proportion of physicians and nursing staff among employees from the three hospitals. Participants were provided with information concerning the purpose of the interview, anonymity and confidentiality conditions, and the voluntariness of participation before each interview. Participants were asked for permission to tape-record the interviews. Informed written consent was acquired. Interviewees were HCWs from different wards and with different professions. Recruitment was performed by the first author and continued until saturation occurred (Patton, 1990). Due to time constraints on the side of the HCWs, interviews were short and lasted approximately ten minutes. The interviewer had no dual relationship with the interviewees. The Research Ethics Board of 
the Faculty of Psychology and Neuroscience at Maastricht University reviewed and approved the study.

Table 4.1 Sample demographics and characteristics of German, Dutch and Belgian HCWs

\begin{tabular}{lllcc}
\hline & Total (N=123) & $\begin{array}{l}\text { German HCWs } \\
\mathbf{( N = 3 1 )}\end{array}$ & $\begin{array}{l}\text { Dutch HCWs } \\
(\mathbf{N}=\mathbf{4 5})\end{array}$ & $\begin{array}{c}\text { Belgian HCWs } \\
\mathbf{( N = 4 7 )}\end{array}$ \\
\cline { 2 - 4 } Immunizers & $50(41 \%)$ & $15(48 \%)$ & $9(20 \%)$ & $26(55 \%)$ \\
Non-immunizers & $73(59 \%)$ & $16(52 \%)$ & $36(80 \%)$ & $21(45 \%)$ \\
Gender & & & & \\
Male & $40(32 \%)$ & $9(29 \%)$ & $18(40 \%)$ & $13(28 \%)$ \\
Female & $83(68 \%)$ & $22(71 \%)$ & $27(60 \%)$ & $34(72 \%)$ \\
Mean age (SD) & $37.11(11.32)$ & $32.77(8.39)$ & $36.64(11.64)$ & $40.40(11.85)$ \\
Occupation & & & & $7(15 \%)$ \\
Physician & $32(26 \%)$ & $6(19 \%)$ & $19(42 \%)$ & $19(40 \%)$ \\
Nurse & $57(46 \%)$ & $14(45 \%)$ & $24(53 \%)$ & $2(5 \%)$ \\
Student & $7(6 \%)$ & $5(17 \%)$ & 0 & $19(40 \%)$ \\
Others & $27(22 \%)$ & $6(19 \%)$ & $2(5 \%)$ & \\
\hline
\end{tabular}

Note: $S D$, standard deviation; HCWs, health care workers

\section{Data collection and analysis}

Data were collected by means of semi-structured interviews. Questions covered the topics a) general information (i.e., What is your position in this hospital?), b) immunization status and reasons for vaccination (i.e., Did you get vaccinated against influenza in the past season?; What are the reasons why you did (not) get vaccinated against influenza in the past season?), c) experiences with influenza vaccination (i.e., What are your experiences with influenza vaccination?), d) intention to get vaccinated (i.e., Are you planning to get vaccinated against influenza in the influenza season 2012/13?), and e) patient advice (i.e., Would you recommend influenza vaccination to your patients and why?). Data were processed with QSR NVivo 8.0 (Doncaster, Australia). The content analysis was based on a combination of a deductive and a general inductive approach (Elo \& Kyngäs, 2008). The deductive analysis was based on concepts of the Reasoned Action Approach (Fishbein \& Ajzen, 2010). Content analysis was conducted by a single coder. No formal testing of reliability could be performed. However, the authors discussed and agreed on the interpretation of the data. Following analysis, quotes were selected on the basis of their representativeness for the findings and subsequently translated from German and Dutch into English. This qualitative study adheres to the RATS guidelines for reporting qualitative studies (Clark, 2003). 


\section{RESULTS}

Interviews were completed with 47 Belgian HCWs of which 26 were vaccinated (10 men and 16 women) and 21 were not vaccinated against influenza ( 3 men and 18 women ), 45 Dutch HCWs of which 9 were vaccinated (4 men and 5 women) and 36 were not vaccinated against influenza (14 men and 22 women), and 31 German HCWs of which 15 were vaccinated against influenza ( 6 men and 9 women) and 16 were not vaccinated against influenza ( 3 men and 13 women; see Table 4.1).

Inspection of the individual wards participating HCWs were working on did not show any noticeable distinction with regard to vaccination status of HCWs working on high risk vs. low risk wards. In the following, we will refer to the vaccinated participants as immunizers and the unvaccinated as non-immunizers. Results for immunizers and non-immunizers are described separately to detect differences between those two groups, in combination with similarities and differences among HCWs from different countries. See Table 4.2 for a detailed overview of the given answers that are summarized below.

\section{Social cognitive reasons}

\section{Reasons of immunizers to get vaccinated}

Belgian, Dutch and German immunizers all reported self-protection, patient protection and protection of family members as most important reasons to get vaccinated against influenza. Across the different countries, self-protection was reported most often.

"First of all to protect myself and to protect my family, that I don't take germs home. But of course also to protect patients [...]."

Other mentioned reasons that were similar across countries were feelings of pressure to get vaccinated from the occupational physician, their employer, the head of the department, or generally the ward they were working on.

"[...] the occupational physician basically insists on doing it. It is strongly recommended to do it. It is voluntary but you are explicitly made attentive that it would be necessary."

Less often reported reasons were having a medical condition that required annual vaccination, having to wear an FFP2 mask at work, having to care for small children or the elderly at home, the protection of fellow colleagues, prevention of workabsenteeism and being pregnant. 


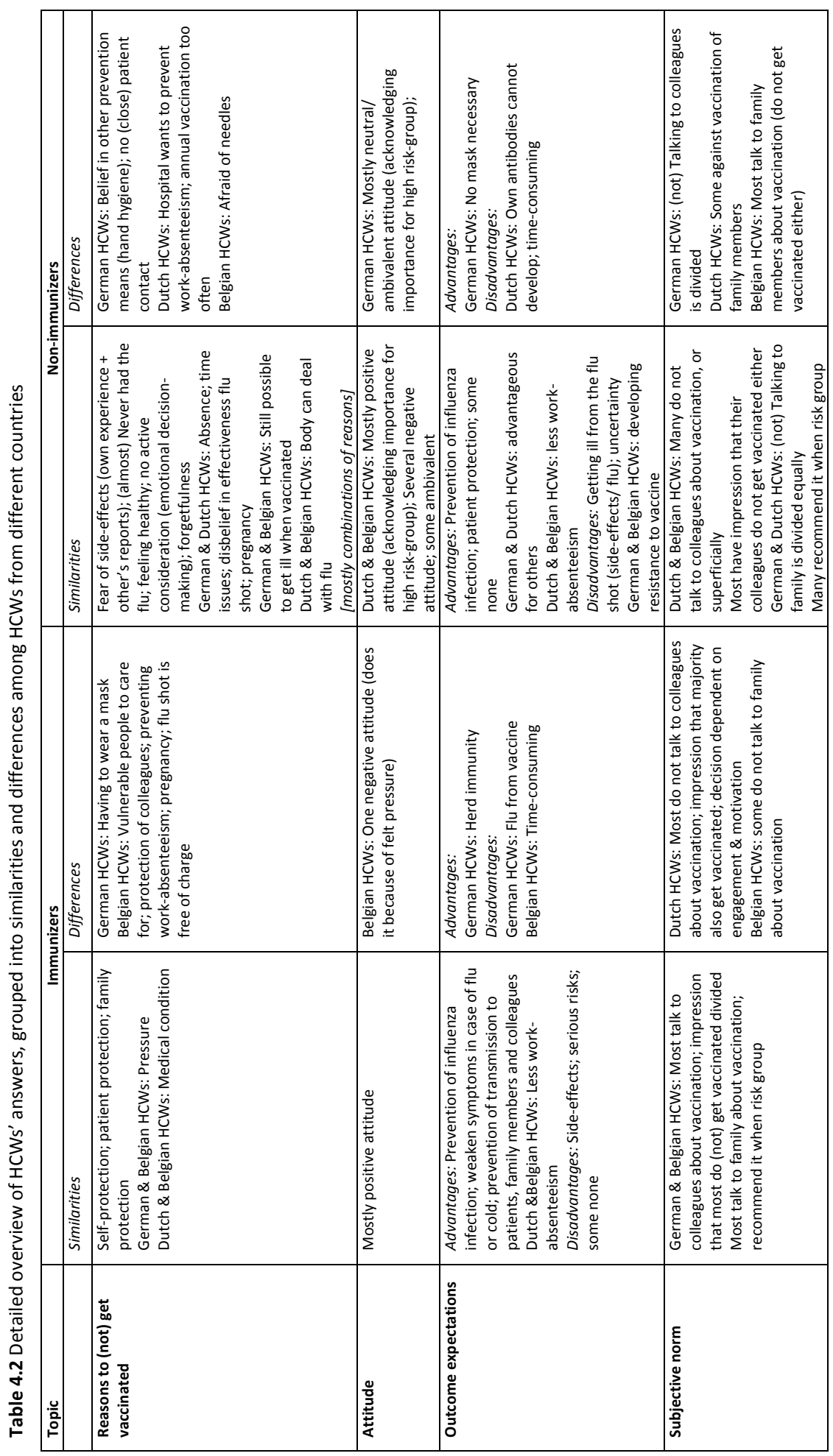




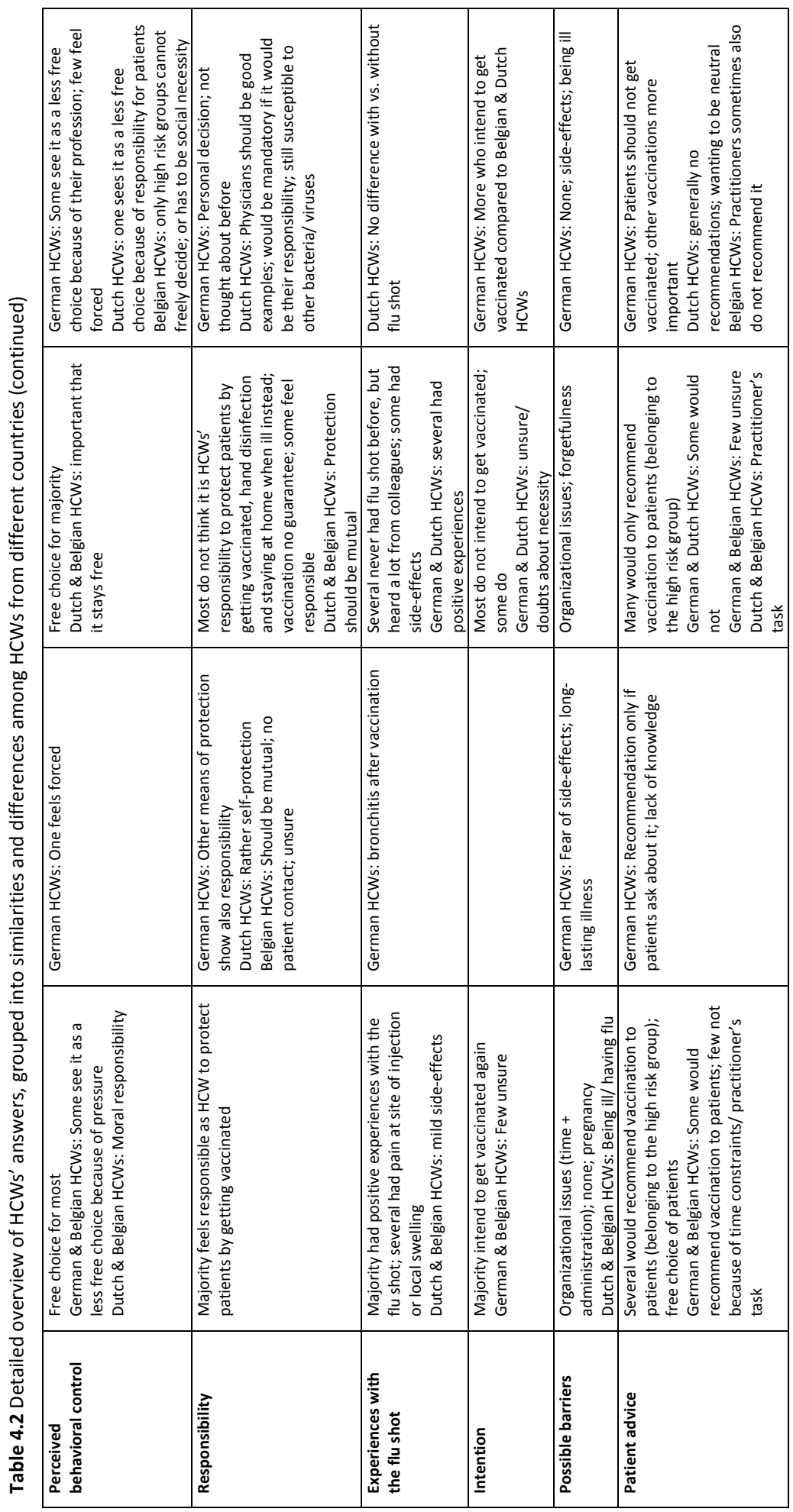




\section{Reasons of non-immunizers to not get vaccinated}

In contrast to immunizers, Belgian, Dutch and German non-immunizers' reasons for not getting vaccinated against influenza can be represented by six categories: Firstly, HCWs who did not get vaccinated the past season, mostly reported that they were afraid of side-effects that the flu shot might have, in particular flu-like symptoms. Some of these fears came from own experience and some came from experiences that others had reported.

"Well, I got vaccinated before and then I was ill with the flu. I had to stay at home for two weeks with real flu symptoms. And since then I said 'No, I don't want it anymore.'."

Secondly, non-immunizers reported that they did not feel at risk to get the flu since they never or almost never had the flu before and reported to feel healthy.

"I never got vaccinated (against influenza) and I never had the flu. So, yeah."

Thirdly, the disbelief in the effectiveness of influenza vaccination in protecting themselves or patients was reported as a reason to not get vaccinated.

"I'm uncertain whether it (influenza vaccination) is working or not."

The fourth category of reasons to not get vaccinated against influenza comprised organizational barriers.

"[...] the reason I didn't do it this season was that the appointments were set on weird times and then I couldn't make it [...]"

Lack of knowledge and misconceptions about who should get vaccinated, the belief that a person benefits from undergoing illness and that there are other protective measures that are more effective in preventing the flu were mentioned as a fifth category.

"[...] And yes, maybe I also thought that because of the pregnancy, I wasn't really sure about it (flu shot), even though the occupational physician said it (that it is recommended) [...]"

Finally, several non-immunizers reported undefined negative emotions or fear resulting from the decision whether to get vaccinated.

"I do think about it, but in the end it's a calculation of - I don't know what rather emotional arguments that tell you, okay it is going well without it (flu shot)." 


\section{Attitude and outcome expectations of immunizers}

Most immunizers reported a positive attitude towards influenza vaccination. Positive outcome expectations originated from the belief in the effectiveness of the flu shot to prevent infections and transmission of influenza to patients and family members.

"Advantages... I mainly see them for my patients. That I won't transmit flu. So actually as a protection for others. For me personally, it doesn't really make a difference. At my age."

Additionally, creating herd-immunity, but also preventing work-absenteeism was seen as an advantage.

"I can't permit getting ill with my job. So if that can be prevented, why not?"

Negative outcome expectations were possible side-effects or more serious consequences that might result from vaccination, but also the local pain that can be associated with an injection.

"The flu shot can have side effects. I experienced it myself that you feel ill immediately after vaccination, feeling shivery."

\section{Attitudes and outcome expectations of non-immunizers}

Among the non-immunizers, Belgian and Dutch HCWs reported a positive attitude towards influenza vaccination, while German HCWs were rather ambivalent. All nonimmunizers acknowledged the importance of influenza vaccination for high risk groups.

"I do have a positive attitude towards it (influenza vaccination). For people that are as healthy as I am, it's not necessary. But of course for babies and the elderly, or women who are pregnant, or people who are really ill, it is beneficial."

Positive and negative outcome expectations resembled those of the immunizers. However, additional disadvantages were mentioned to be the development of a resistance to the vaccine and that there would be no guarantee for the effectiveness of the flu shot because of annual mutations of the influenza virus.

"[...] you never know how the substance itself will affect you. How you tolerate it and you also don't know when you get vaccinated against the flu there are always different types every year - if this, exactly this type is included in the vaccine." 


\section{Subjective norms of immunizers}

With respect to subjective norms, Belgian and German immunizers tended to talk to their colleagues about influenza vaccination, while Dutch immunizers largely did not.

"Yes, we are talking about it every year on the ward, when it's autumn and a flu epidemic is coming."

Belgian and German HCWs reported being uncertain about how many of their colleagues get vaccinated against influenza, while Dutch HCWs thought that the majority of their colleagues would also get immunized.

"In my view, most people go get it (influenza vaccination). They are in favor of it, yes."

Talking to family members about influenza vaccination was common among all immunizers.

"Exactly, I also encourage my family to get vaccinated, because I think that there is also a heightened risk for flu because of me."

\section{Subjective norms of non-immunizers}

Of the non-immunizers, Belgian and Dutch HCWs reported to not talk to their colleagues about influenza vaccination or only very superficially, whereas German HCWs partially talk to their colleagues about it.

"Geez, coincidently it is talked about (influenza vaccination), but it isn't gone into much. No, little attention is paid to that."

Nevertheless, the impression of most non-immunizers was that most colleagues do not get vaccinated against influenza either.

"I think that a big part - at least of my colleagues - is against it, yes."

Talking with family members was only common among Belgian non-immunizers. Some Belgian participants mentioned that family members did not get vaccinated either.

\section{Perceived behavioral control of immunizers}

With respect to perceived behavioral control, most immunizers reported to feel that it is a free choice whether to get vaccinated against influenza. However, the perception that it is part of an occupational obligation and that there is some pressure to get vaccinated (i.e., from the occupational physician), made the decision not entirely free for some immunized HCWs. 
"Er yes, but you also have a responsibility in a hospital for your patient population. So there's a chance there will be pressure to do it from outside, because of that and as long as it doesn't involve problems for me - that I cannot tolerate it or something - yes okay, then it is part of my job to have mandatory vaccinations."

\section{Perceived behavioral control of non-immunizers}

German and Dutch non-immunizers reported the same perception with regard to their freedom to choose whether to get vaccinated as immunizers, whereas all Belgian nonimmunizers reported feeling completely free to choose. Freedom of choice was reported to be important for HCWs.

"Yes, it has to be my own decision. I just heard that you can be obligated in particular work environments. But I don't think that that's okay. It has to be out of free will. Yes."

\section{Responsibility}

\section{Responsibility of immunizers}

The majority of immunizers reported that they feel responsible as HCW to protect patients by getting vaccinated against influenza.

"When I walk around here being ill with the flu, it's also not good for our patients, of course. I mean, the people are already ill and when they then get flu in addition to that, that's never good."

Some German HCWs reported that vaccination is not the only means of protection, respectively that responsibility for patients can also be taken through other measures.

"Yes, but we otherwise have to wear a mask. [...] For me it doesn't make a difference if I'm vaccinated or if I wear a mask. Patients are protected anyway."

Direct patient contact and wanting to prevent work-absenteeism were reported to additionally increase feelings of responsibility.

\section{Responsibility of non-immunizers}

In contrast to the immunizers, many non-immunizers did not feel responsible to get vaccinated against influenza to protect patients. Reasons for not feeling responsible to protect patients by getting vaccinated were the belief that regular hand disinfection 
and staying at home when ill are equally or more effective means of protecting patients against influenza.

"Responsibility for patients, to get vaccinated? Against the flu? No, because when I have it (flu), I stay at home. I take that responsibility."

Moreover, it was reported that vaccination would not guarantee the protection of patients and can therefore not be the responsibility of HCWs and that patients would still be susceptible to other bacteria or viruses that HCWs cannot protect their patients from.

"It is my responsibility to protect patients. But being vaccinated or not, I can still get the flu. So you don't solve anything with a vaccine. So in terms of that, I don't feel responsible for it."

Moreover, a lack of reciprocity, or that patients should be equally accountable to protect HCWs by getting vaccinated decreased feelings of responsibility. One participant concluded that it is not the responsibility of HCWs to get vaccinated for patient safety, because it would otherwise be mandatory in hospitals.

\section{Experiences with the flu shot}

\section{Experiences of immunizers}

The majority of immunizers reported positive experiences with the flu shot in the past, with - if anything - local pain at the site of injection as a negative aspect.

"When I had something it was local, that you have a local pain for one or two days. But I think you have that with every vaccination."

Few participants reported the experience of mild side-effects, such as having a cold after getting vaccinated against influenza.

\section{Experiences of non-immunizers}

Many non-immunizers never got vaccinated against influenza before. The experiences of those that had been vaccinated before differed. Some experiences were positive, while some non-immunizers reported that they had experienced side-effects, ranging from a fever to flu-like symptoms and upper respiratory infections.

"Ahem, I got vaccinated on duty and then I was ill for two days, even though I maybe got ill once in 25 years. So it was actually a negative experience. That's why I say no for the time being." 
Some non-immunizers had gotten flu irrespective of being vaccinated or not and therefore said that they did not experience a difference with or without influenza vaccination.

\title{
Motivation and barriers
}

\section{Motivation and barriers of immunizers}

The intention to get vaccinated again was reported by the majority of immunized HCWs. Possible barriers of converting this intention into action were mostly organizational issues, such as time pressure and administrative barriers that might prevent them from getting the flu shot.

\begin{abstract}
"Well, that's actually mostly in terms of planning. Or at that moment, I know that last time, fortunately there was a second round or something. Because I think I was on duty and then, or there were very specific hours and we had a meeting... Simply in terms of planning."
\end{abstract}

Being ill at the time that vaccination appointments are offered and fear of side-effects were also mentioned as barriers.

"At the time that you are ill, you are not allowed to get it (flu shot) I think. But that's the only one (barrier) I think."

One misconception that was reported by some immunizers was that pregnancy would be a barrier against getting vaccinated.

"Pregnant women. I don't know for sure, I would have to look up if they are allowed to get vaccinated or not."

\section{Motivation and barriers of non-immunizers}

Among Belgian and Dutch non-immunizers, most did not intend to get vaccinated against influenza in the next season, whereas this was rather divided among German HCWs.

"No, I'm not planning on doing it. No."

Organizational issues were also reported barriers by non-immunizers, together with forgetfulness, illness, and fear of side-effects.

"Well, what I said, because you don't know how you will react to the vaccination." 


\section{Patient advice}

\section{Patient advice of immunizers}

Participants were asked if they would recommend influenza vaccination to their patients. German and Dutch immunizers mostly reported that they would recommend influenza vaccination to their patients, especially if they belong to the risk group.

"I don't recommend it to every patient, patients that have a heightened risk: immunocompromised patients, patients with lung diseases, patients aged 65 years and older. To them I do recommend it strongly."

Only some Belgian immunizers would recommend the flu shot. The belief that vaccination is a free decision and that one can only inform people about the benefits, but that it is the responsibility and free choice of the patients was present among most immunizers.

"Not really my responsibility. They still decide that themselves. But you can tell your opinion and the advice, the recommendation to do it."

Time constraints and thinking that it is the task of the responsible practitioner to recommend vaccination were additional reasons to not advice patients about influenza vaccination. All immunized physicians reported to advice influenza vaccination to patients belonging to the risk group, while some nurses said that they would not recommend it.

\section{Patient advice of non-immunizers}

In many cases, non-immunizers would also recommend influenza vaccination, if their patients belong to the risk group.

"Yes, only the patients that really need it, such as people with a cardiac valve, weak condition, those I would (recommend it), the elderly, yes."

However, some non-immunizers said that they would not recommend vaccination.

"No I wouldn't. But that's just because the topic isn't that present in my head, that I would go and address it during my work."

Duties that were perceived as being more important and not being the responsible practitioner were again reasons to not advice vaccination. Non-immunized physicians were in general more willing to advice patients about vaccination than nurses. 


\section{DISCUSSION}

The aim of this study was to explore the social cognitive variables and beliefs associated with the decision to get vaccinated of HCWs in Belgium, Germany and the Netherlands with interviews to gain a direct and more in-depth understanding of these determinants and to identify beliefs that have not been captured by previous quantitative studies.

Belgian, Dutch and German immunizers all reported self-protection, patient protection, and protection of family members as most important reasons to get vaccinated against influenza. It has been suggested before, that the realistic assessment of potential benefits of influenza vaccination for especially the self, but also for others are crucial motivating factors for HCWs to get vaccinated (Hakim, Gaur, \& McCullers, 2011; Smedley et al., 2007). In contrast to that, Belgian, Dutch and German non-immunizers' reasons to not get vaccinated against influenza can be clustered into six categories: 1 ) fear of side effects or illness caused by the vaccine, 2) a low risk perception, 3) the disbelief in the effectiveness of influenza vaccination, 4) organizational barriers, 5) misconceptions/ lack of knowledge, and 6) undefined negative emotions or fear resulting from the decision whether to get vaccinated. These reasons to not get vaccinated are in line with findings from previous empirical and review studies (Aguilar-Díaz et al., 2011; Cohen \& Casken, 2012; Hofmann et al., 2006; Hollmeyer et al., 2009; Looijmansvan den Akker et al., 2009; Smedley et al., 2007; Van den Dool et al., 2008).

The attitude towards influenza vaccination was generally positive, however outcome expectations seemed to influence the decision whether to get vaccinated. With regard to subjective norms, HCWs might be less influenced by what they talk about with colleagues (injunctive norm), than by what they think that colleagues would do (descriptive norm). HCWs that did get vaccinated themselves had the impression that most of their colleagues also get vaccinated, while the opposite was true for nonimmunizers. In contrast, Looijmans-van den Akker and colleagues (2009) had found a significant association of injunctive subjective norms with vaccination uptake. Moreover, immunized HCWs had more and better experiences with the flu shot, felt more responsible to protect patients by getting vaccinated and intended to get vaccinated against influenza again. Previous influenza vaccination uptake had been shown to be a main predictor of future vaccination uptake (Aguilar-Díaz et al., 2011; Hollmeyer et al., 2009). For some non-immunizers, feelings of responsibility to protect patients seemed to be associated with feelings of reciprocity, or the expectation that patients should be equally accountable to protect HCWs by getting vaccinated. Reciprocity has been suggested to be an important factor influencing altruistic behavior (Gintis, Bowles, Boyd, \& Fehr, 2003; Jones, 2002). With respect to perceived behavioral control, most HCWs reported to feel that it is a free choice whether to get vaccinated against influenza. Freedom of choice has been shown to be highly valued even by HCWs that get vaccinated against influenza (Hakim et al., 2011). 
Among the reasons that influenced the decision to not get vaccinated, we identified three additional beliefs that have not been extensively described in the literature before. Firstly, a reason to not get vaccinated was the belief that other means of prevention, such as regular hand disinfection or staying at home when ill are as effective or even more effective in preventing influenza transmission to patients than influenza vaccination (Bridges, Kuehnert, \& Hall, 2003). Secondly, some immunizers showed an omission bias, which is the preference to not get vaccinated over getting vaccinated if one thinks that vaccination could cause illness (Baron \& Ritov, 2004). The omission bias was previously associated with parent's decision to not vaccinate their children (Asch et al., 1994). Finally, other health beliefs surfaced that comprise the belief that it is better for one's health to undergo illness and to build own antibodies during illness than to prevent illness by getting vaccinated, which is related to a previously described belief that vaccination would weaken the immune system (Van den Dool et al., 2008).

Furthermore, HCWs recommend influenza vaccination to patients, when they belong to the risk group. However, vaccination was seen as a free choice, not only for $\mathrm{HCWs}$, but also for patients. The question was hypothetical in nature and it seemed that immunizers, as well as non-immunizers were very rarely asked about influenza vaccination by patients and did not talk about vaccination with their patients due to other topics and duties that were perceived as more important. Moreover, it was reported that only authorized practitioners would be allowed to give advice about medication and vaccination, which means that it is not a part of the duties of most of the participants in this study. Accordingly, more immunized and non-immunized physicians were willing to advice influenza vaccination to patients than immunized and nonimmunized nursing staff.

This study extends current knowledge about social cognitive variables and beliefs that affect the motivation of HCWs to get vaccinated against influenza. The study was conducted in three European countries, one of them not having been systematically surveyed before (Belgium). The present study used a qualitative research method, which has the advantage of gaining a direct and in-depth understanding of the beliefs underlying the decision to get vaccinated against influenza of HCWs (Fishbein \& Ajzen, 2010). However, due to cross-sectional analysis no causal relationships could be established, nor the relative importance of social cognitive variables and beliefs in explaining why HCWs get or do not get vaccinated against influenza. Future research should use the insights of this study and quantify the results. Moreover, future studies should explore the predictive value of the social cognitive variables and beliefs found in this study in explaining the intention to get vaccinated. Secondly, coding of the interviews was performed by only one coder, which made inter-rater reliability not possible. This could have biased the results. Qualitative research is inherently interpretive and more coders could potentially decrease bias. However, coding was done in a systematic way by developing a coding scheme and all authors discussed the analysis process and interpretation of the data extensively so as to reduce bias to a minimum. Thirdly, few 


\section{Chapter 4}

participants belonged to a high risk group $(\mathrm{N}=5)$, due to age, pregnancy or a medical condition. We did not exclude them, however it should be noted that their reasons for getting vaccinated against influenza could be related to their condition, rather than their occupation. Finally, the participating hospitals were chosen based on convenience, rather than representativeness of hospitals in the three different countries. Therefore, generalizations to the collective population of Belgian, Dutch and German HCWs should be treated with caution.

\section{CONCLUSIONS}

The reasons that HCWs have for getting vaccinated against influenza (or not) seem to be similar in Belgium, Germany and the Netherlands. This was also true for the social cognitive variables that are believed to drive the intention to get vaccinated, even though some differences surfaced. A quantitative investigation of those social cognitive variables is needed in order to determine the importance of the social cognitive variables in explaining the intention to get vaccinated and the importance of the similarities and differences between countries that have been found in this study. This would in turn shed more light onto the question whether intervention programs developed to increase vaccination uptake, have to be country-specific or if one intervention program can be used in different countries. 


\section{CHAPTER 5}

Social-cognitive predictors of health care workers' intention to get vaccinated against influenza in Belgian, Dutch and German hospital settings

Based on:

Lehmann, B. A., Ruiter, R. A. C., van Dam, D., Wicker, S., \& Kok, G. (2014). Sociocognitive predictors of the intention of healthcare workers to receive the influenza vaccine in Belgian, Dutch and German hospital settings. Journal of Hospital Infection, 89, 202209. 


\section{Chapter 5}

\section{ABSTRACT}

Influenza vaccination of health care workers (HCWs) is recommended to prevent the transmission of influenza to vulnerable patients. Nevertheless, vaccination coverage rates of HCWs in European countries have been low. The aim of this study was to investigate the relative and combined strength of social cognitive variables from past research, theory and a qualitative study in explaining the motivation to obtain influenza vaccination. An anonymous, online questionnaire was distributed among HCWs in hospital settings in Belgium, Germany and the Netherlands between February and April 2013. The findings showed that attitude and past vaccination uptake explain a considerable amount of variance in the intention to get vaccinated against influenza. Moreover, low perceived social norms, having an omission bias, low moral norms, being older, having no patient contact, and being Belgian or Dutch compared to being a German HCW increased the probability of no intention to get vaccinated compared to being unsure about vaccination. A high intention was shown to be more likely than being unsure about vaccination for HCWs with a high perceived susceptibility to contract influenza, low naturalistic views, and a lower motivation to solely get vaccinated for self-protection. Country-specific interventions and a focus on different social cognitive variables depending on whether HCWs have the intention to get vaccinated against influenza or not may be beneficial to promote vaccination uptake. 


\section{INTRODUCTION}

Influenza is a major public health problem causing severe morbidity and mortality in high risk groups (Mertz et al., 2013; WHO, 2005). Previous research has shown that vaccination of health care workers ( $\mathrm{HCWs)} \mathrm{reduces} \mathrm{all-cause} \mathrm{mortality} \mathrm{in} \mathrm{long-term}$ care patients by up to $29 \%$ (Ahmed, Lindley, Allred, Weinbaum, \& Grohskopf, 2014; Dolan et al., 2012; WHO, 2005), and might have a similar or an even higher impact on patients in acute care settings (Amodio et al., 2014; Bénet et al., 2012; Macesic, Kotsimbos, Kelly, \& Cheng, 2013; Van den Dool, Bonten, Hak, \& Wallinga, 2009). Health Authorities therefore recommend the vaccination of HCWs (CDC, 2011; WHO, 2009). Nevertheless, vaccination coverage rates of HCWs in European countries have been low, ranging from 6.4-26.3\% (Blank, Schwenkglenks, \& Szucs, 2009; Endrich, Blank, \& Szucs, 2009; Guthmann et al., 2012).

Recently different intervention programs to increase influenza vaccination rates among HCWs have been developed (Doratotaj, Macknin, \& Worley, 2008; Llupià et al., 2010; Looijmans-van den Akker et al., 2010). However, these programs show at most small effects on vaccination behavior and their long-term success is unknown. Kok et al. (2011) suggested that a systematic approach (i.e., Intervention Mapping) is needed for the successful development and implementation of programs to promote influenza vaccination among HCWs, starting with a detailed analysis of the problematic behavior and identifying social cognitive variables that drive the recommended behavior.

We recently conducted individual semi-structured interviews with HCWs in Belgium, Germany and the Netherlands $(N=123)$ to obtain an in-depth understanding of the reasons to get the influenza vaccination or not, and to gain input for the development of the survey instrument used in the present study. The results reflected most of the findings that have been previously reported in review studies on drivers of influenza vaccination (Aguilar-Díaz, Jiménez-Corona, \& Ponce-de-León-Rosales, 2011; Cohen \& Casken, 2012; Hollmeyer, Hayden, Poland, \& Buchholz, 2009). Positive associations with acceptance of influenza vaccination were found for motivation to protect oneself, willingness to protect patients, family members and/or colleagues, positive perceived norms towards vaccination, perceived moral obligations to get vaccinated, and the capacity to receive the vaccine conveniently; whereas low perceived susceptibility to contract influenza, low perceived severity of influenza, disbelief in the relevance of influenza vaccination and the supporting scientific evidence were reasons not to obtain the vaccination. Being older and being a physician as opposed to a nurse contributed to higher acceptance of influenza vaccination as well as previous vaccine receipt (Aguilar-Díaz et al., 2011; Cohen \& Casken, 2012; Hollmeyer et al., 2009).

In addition to these variables, three additional beliefs were identified that negatively influenced the decision to obtain influenza vaccination: the omission bias, naturalistic beliefs and prevention beliefs. Omission bias is the preference of inaction, when action might cause harm and has previously been associated with parent's decision to 
not vaccinate their children (Asch et al., 1994). Naturalistic beliefs comprised that it is better for one's health to undergo illness and build antibodies during illness, than preventing illness by getting vaccinated. Prevention beliefs entail different means of prevention (e.g., regular hand disinfection, staying at home when ill) that are seen as being as effective, or more effective than getting vaccinated in preventing influenza (Bridges, Kuehnert, \& Hall, 2003).

The relative strength of these and other identified variables in explaining the motivation to obtain influenza vaccination among HCWs in hospital settings in Belgium, Germany and the Netherlands is not clear. In the present study, we therefore conduct a cross-sectional survey to assess the relative and combined strength of the previously identified social cognitive variables and three additional beliefs in explaining the intention of HCWs. Moreover, the qualitative study suggested differences between neighboring countries in the variables influencing the intention to get vaccinated against influenza. This might point to the importance of a country-specific intervention development in the future.

\section{METHODS}

\section{Participants and procedure}

A cross-sectional study was performed between February-April 2013 in 20 hospitals in Belgium, Germany and the Netherlands. Hospitals were contacted via phone first and subsequently were sent detailed information about participation in the study via email or letter, if requested. In Belgium, 24 hospitals in 19 cities were approached, resulting in 7 participating hospitals (29\%). In Germany, 33 hospitals in 16 cities were contacted, resulting in 7 participating hospitals (21.2\%). In the Netherlands, 21 hospitals in 14 cities were contacted, resulting in participation of 6 hospitals (28.6\%). Included hospitals provided a contact person (e.g., the occupational physician) who agreed to distribute an email to several wards, respectively all employees of a hospital, with information on the study and a link to the online survey. Approximately three weeks later, a second email was sent to the contact person with the request to forward this reminder to hospital employees, in case they had not yet participated. For anonymity reasons we did not ask participants about the hospital and department they are working in.

\section{The online survey}

The online survey consisted of 80 questions targeting social cognitive variables and additional beliefs about annual influenza vaccination, past behavior and experiences, and socio-demographics. Variables were measured on 7-point Likert scales ranging from 1 = totally disagree to 7 = totally agree, unless otherwise indicated. Items measuring the same underlying theoretical construct were averaged into one single con- 
struct when internal consistency was sufficient (Cronbach's alpha $\alpha>.60$ or Pearson correlation coefficient $r>.50$ ). Table 5.1 provides an overview of the constructs and their internal consistency.

Table 5.1 Overview of constructs measured by the online survey

\begin{tabular}{|c|c|c|c|}
\hline Variable & $\begin{array}{l}\text { Number } \\
\text { of items }\end{array}$ & Reliability & Example questions \\
\hline Intention & 2 & $r=.97$ & I intend to get vaccinated against influenza annually. \\
\hline Attitude & 6 & $\alpha=.92$ & $\begin{array}{l}\text { Getting vaccinated against influenza annually is: very good } \\
\text { - very bad; comforting - frightening. }\end{array}$ \\
\hline Subjective Norm & 4 & $\alpha=.70$ & $\begin{array}{l}\text { Most of my colleagues get vaccinated against influenza } \\
\text { annually. }\end{array}$ \\
\hline Perceived severity & 2 & $r=.37$ & $\begin{array}{l}\text { Influenza is a serious infection that can lead to } \\
\text { complications. }\end{array}$ \\
\hline Perceived susceptibility & 3 & $\alpha=.68$ & $\begin{array}{l}\text { I am healthy, therefore I don't need to get vaccinated } \\
\text { against influenza annually. }\end{array}$ \\
\hline Capacity & 1 & n.a. & $\begin{array}{l}\text { I am confident that I could get vaccinated against influenza } \\
\text { annually (if I want to). }\end{array}$ \\
\hline Autonomy & 1 & n.a. & $\begin{array}{l}\text { Getting vaccinated against influenza annually is } \\
\text { completely up to me. }\end{array}$ \\
\hline Omission bias & 1 & n.a. & $\begin{array}{l}\text { I prefer to get influenza, instead of getting vaccinated } \\
\text { against influenza. }\end{array}$ \\
\hline Prevention beliefs & 3 & $\alpha=.62$ & $\begin{array}{l}\text { By staying at home when I am ill, I can sufficiently protect } \\
\text { patients from getting influenza. }\end{array}$ \\
\hline Naturalistic beliefs & 3 & $\alpha=.80$ & $\begin{array}{l}\text { I think that it is better to undergo influenza, then to get } \\
\text { vaccinated against influenza annually. }\end{array}$ \\
\hline Disbelief science & 2 & $r=.60$ & $\begin{array}{l}\text { As far as I know, there is insufficient scientific evidence } \\
\text { that influenza vaccination is effective in preventing } \\
\text { influenza. }\end{array}$ \\
\hline Disbelief relevance & 3 & $\alpha=.80$ & $\begin{array}{l}\text { I think that the relevance of the annual influenza } \\
\text { vaccination is overestimated. }\end{array}$ \\
\hline Moral norm & 2 & $r=.69$ & $\begin{array}{l}\text { If I would get vaccinated against influenza annually, I } \\
\text { would do it to protect my environment. }\end{array}$ \\
\hline Responsibility & 2 & $r=.62$ & $\begin{array}{l}\text { I think it is the responsibility of health care workers to get } \\
\text { vaccinated against influenza annually. }\end{array}$ \\
\hline Self-protection & 1 & n.a. & $\begin{array}{l}\text { If I would get vaccinated against influenza annually, I } \\
\text { would do it to protect myself. }\end{array}$ \\
\hline Management & 4 & $\alpha=.84$ & $\begin{array}{l}\text { How satisfied are you with the management of this } \\
\text { hospital? very satisfied - not at all satisfied. }\end{array}$ \\
\hline
\end{tabular}

In addition, past vaccination behavior was measured with two questions ('In past years I got vaccinated against influenza, when it was offered to me: 1 = always; 7 = never.'; 'Did you get vaccinated against influenza this year (season 2012/ 2013)? yes/no.'). Past experience with having influenza was measured with two questions ('How often did 
you have influenza in the past? Never/more than 10 times.'; 'Did you have influenza last winter? no/yes, once/yes, more than once.'). Demographic measures were profession (physician/nursing staff/other HCWs with patient contact/non-HCWs with no patient contact), gender, country and age group (younger than 20 years/20-29 years/30-39 years/40-49 years/50-59 years/older than 60 years). Age categories were chosen to ensure anonymity of participants.

\section{Data analysis}

SPSS 19.0 was used to analyze the data. Very few responses had not been properly saved by the online survey program. We took full account of all information available, instead of using imputed values for missing data. Following a descriptive analysis of the sample (frequencies), univariate associations between intention, social cognitive variables and additional beliefs were analyzed with Pearson correlation coefficients. Differences between HCWs from Belgium, Germany and the Netherlands were tested with multivariate analysis of covariance (MANCOVA), while controlling for significant differences among the three samples on demographic and influenza-related characteristics. Intention was shown to be distributed U-shaped and to best be classified into three groups; no intention to get vaccinated against influenza $(0=1.0)$, not having made a clear decision about vaccination (1=1.5-6.5), and a high intention to get vaccinated $(2=7.0)$. Therefore, multinominal logistic regression was used to show the effect of the independent variables on the probability of (1) having no intention to get vaccinated vs. not having made a clear decision and (2) having a high intention to get vaccinated vs. not having made a clear decision. Stepwise multinominal logistic regressions were performed so that the contribution of social cognitive variables (Model 1), additional beliefs (Model 2) and past behavior and demographics (Model 3) in explaining intention could be examined and the differences in classification accuracy demonstrated. The Nagelkerke index was chosen above the Cox and Snell index, because it is an appropriate adjustment, allowing for a maximum value of 1.00 (Cohen, Cohen, West, \& Aiken, 2003).

\section{RESULTS}

\section{Descriptive statistics}

The study sample consisted of 1022 participants working in hospital settings; 298 in Belgium, 206 in Germany, and 518 in the Netherlands (see Table 5.2). Of the participants, 227 were male (22.2\%) and 795 were female (77.9\%). Most participants were between $40-59$ years of age (58.5\%). The sample consisted of 570 nurses $(55.8 \%)$ and 152 physicians (14.9\%); 142 participants (13.9\%) indicated being other HCWs but had patient contact (e.g., paramedics, physiotherapists, dieticians), and 158 (15.5\%) indi- 
cated being no HCWs with no direct patient contact (e.g., administrative workers, microbiologists, management). In total, 379 (37.1\%) participants in the sample got vaccinated in the influenza season 2012/2013 and 195 (19.1\%) indicated having had an influenza-like illness (ILI) in that influenza season.

Chi-square analyses showed that the three samples were different on the measures of gender, age-group, occupation, vaccination status in season 2013-2014, and ILI in season 2013-2014 ( $p s<.05)$.

Table 5.2 Demographics and HCW characteristics

\begin{tabular}{|c|c|c|c|c|}
\hline & $\begin{array}{l}\text { Netherlands } \\
(\mathrm{N}=518,50.7 \%)\end{array}$ & $\begin{array}{l}\text { Belgium } \\
(\mathrm{N}=298,29.2 \%)\end{array}$ & $\begin{array}{l}\text { Germany } \\
(\mathrm{N}=206,20.1 \%)\end{array}$ & Total $(\mathrm{N}=1022)$ \\
\hline \multicolumn{5}{|l|}{ Gender } \\
\hline Male & $84(16.2)$ & $66(22.1)$ & $77(37.4)$ & $227(22.2)$ \\
\hline Female & $434(83.8)$ & $232(77.9)$ & $129(62.6)$ & $795(77.8)$ \\
\hline \multicolumn{5}{|l|}{ Age-group } \\
\hline$<20$ years & & & $1(0.5)$ & $1(0.1)$ \\
\hline $20-29$ years & $83(16)$ & $47(15.8)$ & $25(12.1)$ & $155(15.2)$ \\
\hline $30-39$ years & $101(19.5)$ & $69(23.2)$ & $51(24.8)$ & $221(21.6)$ \\
\hline $40-49$ years & $146(28.2)$ & $76(25.5)$ & $74(35.9)$ & $296(29)$ \\
\hline $50-59$ years & $158(30.5)$ & 95 (31.9) & $48(23.3)$ & $301(29.5)$ \\
\hline$>60$ years & $28(5.4)$ & $11(3.7)$ & $5(2.4)$ & $44(4.3)$ \\
\hline \multicolumn{5}{|l|}{ Occupation } \\
\hline Physician & $48(9.3)$ & $21(7)$ & $83(40.3)$ & $152(14.9)$ \\
\hline Nursing staff & $277(53.5)$ & $206(69.1)$ & $87(42.2)$ & $570(55.8)$ \\
\hline Other HCWs, patient contact & $98(18.9)$ & $36(12.1)$ & $8(3.9)$ & $142(13.9)$ \\
\hline Non-HCWs, no patient contact & $95(18.3)$ & $35(11.8)$ & $28(13.6)$ & $158(15.5)$ \\
\hline Vaccinated in season $2012-2013$ & $146(28.2)$ & $158(53)$ & $75(36.4)$ & $379(37.1)$ \\
\hline ILI in season $2012-2013$ & $107(20.7)$ & $35(11.7)$ & $53(25.7)$ & $195(19.1)$ \\
\hline
\end{tabular}

Data are reported as number of participants (\%).

Correlates of intention and differences between HCWs from different countries

Table 5.3 shows that all social cognitive variables and additional beliefs were significantly correlated with intention to get vaccinated against influenza. A small effect is $r=$ .10-.23, a moderate effect $r=.24-.36$ and a large effect is $r \geq .37$ (Cohen et al., 2003). We found strong positive univariate associations with intention for attitude, perceived norm, perceived susceptibility, moral norm, responsibility as HCWs, and past vaccination frequency. Strong negative associations with intention were found for the disbelief in the relevance of the flu shot, the disbelief in scientific evidence for effectiveness, for holding naturalistic views, and having an omission bias. 


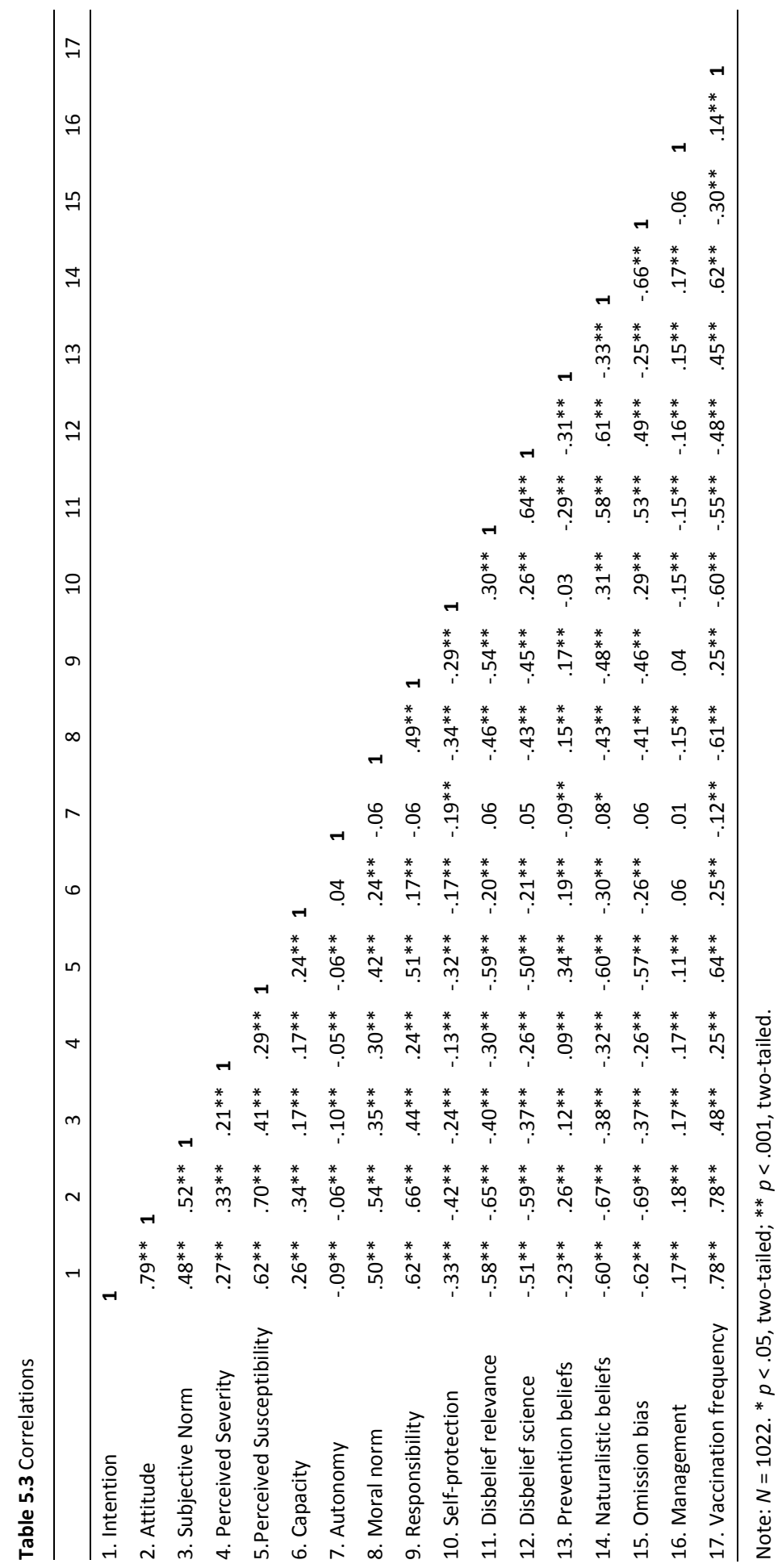


Table 5.4 presents the means and standard deviations for Belgian, German and Dutch HCWs on the social cognitive variables and additional beliefs and the results of the MANCOVA testing differences between the three countries, while controlling for the significant differences on gender, age-group, occupation, vaccination status in season 2013-2014, and ILI in season 2013-2014 among the three samples. In comparison with Dutch and German HCWs, Belgian HCWs had a significantly higher intention, more satisfaction about the hospital management, and felt more responsibility to get vaccinated. Dutch HCWs had significantly lower scores on attitude, perceived norm, perceived susceptibility, moral norm, capacity, autonomy, and vaccination frequency than German and Belgian HCWs. Moreover, disbelief in the relevance of the flu shot and omission bias scored significantly higher among Dutch compared to German and Belgian HCWs. Perceived severity of influenza, self-protection motive, disbelief in the scientific evidence of the effectiveness of the flu shot and naturalistic beliefs differed significantly among the three countries. Perceived severity was lowest for Dutch and highest for German HCWs. The self-protection motive and naturalistic beliefs were highest among Dutch HCWs and lowest in German HCWs. The disbelief in the scientific evidence was lowest in Belgian and highest in Dutch HCWs.

Table 5.4 Differences between Dutch, Belgian and German HCWs

\begin{tabular}{llllc}
\hline Variable & $\begin{array}{l}\text { Dutch HCWs } \\
\mathbf{( N = 5 1 8 )}\end{array}$ & $\begin{array}{l}\text { Belgian HCWs } \\
(\mathbf{N}=\mathbf{2 9 8})\end{array}$ & $\begin{array}{l}\text { German HCWs } \\
\mathbf{( N = 2 0 6 )}\end{array}$ & F value \\
\hline Intention & $.85(.71)^{2}$ & $1.27(.71)^{1,3}$ & $.99(.78)^{2}$ & $6.776^{* *}$ \\
Attitude & $4.17(.04)^{2,3}$ & $4.65(.06)^{1}$ & $4.71(.06)^{1}$ & $32.207^{* *}$ \\
Subjective norm & $2.79(1.00)^{2,3}$ & $3.66(1.23)^{1}$ & $3.48(1.08)^{1}$ & $46.875^{* *}$ \\
Perceived severity & $5.37(1.05)^{2,3}$ & $5.68(1.03)^{1,3}$ & $5.91(.89)^{1,2}$ & $23.442^{* *}$ \\
Perceived susceptibility & $3.77(1.64)^{2,3}$ & $4.86(1.72)^{1}$ & $4.72(1.69)^{1}$ & $28.053^{* *}$ \\
Capacity & $5.83(1.89)^{3}$ & $6.22(1.53)^{3}$ & $6.48(1.29)^{1,2}$ & $8.616^{* *}$ \\
Autonomy & $6.50(1.10)^{2}$ & $6.57(.96)$ & $6.67(.85)^{1}$ & $3.895^{*}$ \\
Management & $4.53(1.09)^{2}$ & $5.02(1.15)^{1,3}$ & $4.38(1.11)^{2}$ & $19.199^{* *}$ \\
Moral norm & $4.35(1.65)^{2,3}$ & $5.32(1.59)^{1}$ & $5.35(1.48)^{1}$ & $28.806^{* *}$ \\
Responsibility & $4.27(1.84)^{2}$ & $5.24(1.89)^{1,3}$ & $4.43(2.09)^{2}$ & $7.433^{* *}$ \\
Self-protection & $3.57(1.98)^{2,3}$ & $2.63(1.71)^{1,3}$ & $2.29(1.53)^{1,2}$ & $37.345^{* *}$ \\
Disbelief relevance & $4.25(1.36)^{2,3}$ & $3.22(1.53)^{1}$ & $3.50(1.63)^{1}$ & $27.692^{* *}$ \\
Disbelief science & $4.74(1.27)^{2,3}$ & $3.55(1.47)^{1,3}$ & $4.02(1.62)^{1,2}$ & $38.850^{* *}$ \\
Prevention beliefs & $3.84(1.25)$ & $4.05(1.42)$ & $3.94(1.47)$ & 1.157 \\
Naturalistic beliefs & $4.09(1.55)^{2,3}$ & $3.23(1.55)^{1,3}$ & $3.07(1.67)^{1,2}$ & $23.073^{* *}$ \\
Omission bias & $3.67(2.06)^{2,3}$ & $2.52(1.80)^{1}$ & $2.92(2.04)^{1}$ & $13.052^{* *}$ \\
Vaccination frequency & $3.20(2.46)^{2}$ & $4.49(2.59)^{1}$ & $3.72(2.46)$ & $3.139^{*}$ \\
\hline
\end{tabular}

Data are reported as mean (SD). ${ }^{*} p<.05$, two-tailed ; ** $p<.01$, two-tailed.

The superscripts indicate which mean scores in a row differ significantly at $p<.05$

Covariates included in this analysis are gender, age-group, occupation, vaccination status in season 20122013, and ILI in season 2012-2013 


\section{Multinominal logistic regression}

\section{No intention to get vaccinated vs. no clear decision}

The results of the multinominal logistic regression are shown in Table 5.5. Results of the final model are described. HCWs were more likely to have no intention to get vaccinated vs. not having made a clear decision when they reported a more negative attitude and perceived norm towards influenza vaccination, indicated an omission bias and lower moral norm to protect patients by getting vaccinated, had a lower frequency of influenza vaccinations in the past, and were older. Furthermore, other HCWs with patient contact were significantly less likely to have no intention to get vaccinated than non-HCWs with no patient contact. Finally, Dutch and Belgian HCWs were significantly more likely to have no intention to vaccinate than German HCWs.

\section{High intention to get vaccinated vs. no clear decision}

HCWs with a positive attitude towards influenza vaccination, a high perceived susceptibility towards influenza, low naturalistic views, self-protection motives, and satisfaction with the hospital management were significantly more likely to have a high intention to get vaccinated vs. not having made a clear decision. Also, a higher frequency of influenza vaccinations in the past increases the probability to have a high intention to get vaccinated against influenza vs. not having made a clear decision.

The multinominal logistic regression with the social cognitive variables as predictors, showed a classification accuracy of $75.1 \%$ (Pseudo $R^{2}=.73$ ). By adding the additional beliefs as predictors and subsequently past behavior and demographic variables, the classification accuracy increased to $78.8 \%$ (Pseudo $R^{2}=.82$ ). Likelihood ratio tests confirmed a significant contribution of the added predictors (Model 1 vs. Model 2: likelihood ratio $\chi^{2}(18)=87.568, p<.001$; Model 2 vs. Model 3: likelihood ratio $\chi^{2}(18)=$ 166.529, $p<.001$; Model 1 vs. Model 3: likelihood ratio $\chi^{2}(36)=254.097, p<.001$ ).

Additional analyses were conducted to test for interactions between each predictor variable and country. Next to a single significant interaction between perceived severity and country for the comparison of no intention vs. unclear decision ( $p \geq .05)$, no further interactions were detected. Therefore, country was only included as main effect and thus controls for possible effects of country when predicting the contributions of the other predictors. 
Table 5.5 Multinominal logistic regression

\begin{tabular}{|c|c|c|c|c|c|c|c|c|c|c|c|c|c|}
\hline \multirow[b]{2}{*}{ Predictors } & \multirow[b]{2}{*}{$r$} & \multicolumn{4}{|c|}{ Model $1^{\text {a }}$} & \multicolumn{4}{|c|}{ Model $2^{b}$} & \multicolumn{4}{|c|}{ Model $3^{c}$} \\
\hline & & $\mathrm{b}$ & S.E. & Wald & $\mathrm{p}$ & $\mathrm{b}$ & S.E. & Wald & $\mathrm{p}$ & $\mathrm{b}$ & S.E. & Wald & $\mathrm{p}$ \\
\hline \multicolumn{14}{|c|}{ No intention vs. no clear decision $(N=731)$} \\
\hline Attitude & $-.53^{* *}$ & -1.36 & .13 & 114.14 & .000 & -1.11 & .15 & 55.95 & .000 & -.98 & .16 & 37.68 & .000 \\
\hline Subjective Norm & $-.30 * *$ & -.29 & .11 & 6.86 & .009 & -.26 & .12 & 4.96 & .03 & -.30 & .13 & 5.15 & .02 \\
\hline $\begin{array}{l}\text { Perceived } \\
\text { Susceptibility }\end{array}$ & $-.26 * *$ & .03 & .07 & .133 & .72 & .09 & .08 & 1.20 & .27 & .10 & .09 & 1.25 & .26 \\
\hline $\begin{array}{l}\text { Perceived } \\
\text { Severity }\end{array}$ & $-.10^{* *}$ & .03 & .09 & .09 & .76 & .15 & .10 & 2.23 & .14 & .07 & .11 & .44 & .51 \\
\hline Autonomy & $.17^{* *}$ & .35 & .11 & 9.84 & .002 & .30 & .12 & 6.32 & .01 & .23 & .13 & 2.90 & .09 \\
\hline Capacity & -.06 & .07 & .05 & 2.10 & .15 & .08 & .05 & 2.12 & .15 & .06 & .06 & 1.15 & .28 \\
\hline Omission bias & $.40 * *$ & & & & & .25 & .06 & 14.54 & .000 & .23 & .07 & 11.38 & .001 \\
\hline $\begin{array}{l}\text { Naturalistic } \\
\text { beliefs }\end{array}$ & $.31 * *$ & & & & & -.05 & .09 & .26 & .61 & -.03 & .10 & .11 & .74 \\
\hline Disbelief science & $.25^{* *}$ & & & & & -.16 & .10 & 2.34 & .13 & -.16 & .11 & 2.08 & .85 \\
\hline $\begin{array}{l}\text { Disbelief } \\
\text { relevance }\end{array}$ & $.31^{* *}$ & & & & & .11 & .10 & 1.19 & .28 & .19 & .11 & 2.70 & .10 \\
\hline $\begin{array}{l}\text { Prevention } \\
\text { beliefs }\end{array}$ & $-.13^{* *}$ & & & & & -.03 & .08 & .14 & .71 & .00 & .09 & .00 & .98 \\
\hline Moral norm & $-.37 * *$ & & & & & -.27 & .07 & 14.49 & .000 & -.27 & .08 & 12.65 & .000 \\
\hline Responsibility & $-.37 * *$ & & & & & -.19 & .06 & 9.27 & .002 & -.12 & .07 & 2.43 & .12 \\
\hline Self-protection & $.11^{* *}$ & & & & & -.07 & .06 & 1.42 & .23 & .01 & .06 & .04 & .84 \\
\hline Management & $-.13 * *$ & & & & & -.19 & .09 & 4.13 & .04 & -.15 & .10 & 2.23 & .14 \\
\hline Flu last season & -.01 & & & & & & & & & .24 & .15 & 2.61 & .11 \\
\hline $\begin{array}{l}\text { Vaccination } \\
\text { frequency }\end{array}$ & $-.45^{* *}$ & & & & & & & & & -.57 & .10 & 31.96 & .000 \\
\hline Age group & $.08^{*}$ & & & & & & & & & .20 & .10 & 4.46 & .04 \\
\hline Gender & $.08^{*}$ & & & & & & & & & .36 & .29 & 1.56 & .21 \\
\hline Physician & $-.09 *$ & & & & & & & & & -.22 & .46 & .22 & .64 \\
\hline Nursing staff & $.07 *$ & & & & & & & & & -.34 & .30 & 1.81 & .18 \\
\hline $\begin{array}{l}\text { Other HCWs, p. } \\
\text { contact }\end{array}$ & $-.08 *$ & & & & & & & & & -.87 & .39 & 4.97 & .03 \\
\hline $\begin{array}{l}\text { Non-HCWs, no p. } \\
\text { contact }\end{array}$ & . & & & & & & & & & . & . & . & . \\
\hline The Netherlands & .06 & & & & & & & & & -1.47 & .35 & 17.85 & .000 \\
\hline Belgium & $-.14^{* *}$ & & & & & & & & & -1.28 & .37 & 12.05 & .001 \\
\hline Germany & . & & & & & & & & & . & . & . & . \\
\hline
\end{tabular}

a Model 1 refers to a multinominal logistic regression with only the social cognitive variables as predictors. b Model 2 refers to multinominal logistic regression with the social cognitive variables and additional beliefs as predictors.

c Model 3 refers to multinominal logistic regression with the social cognitive variables, additional beliefs, and demographics and past behavior as predictors.

$* p<.05$, two-tailed; ** $p<.01$, two-tailed. 
Table 5.5 Multinominal logistic regression (continued)

\begin{tabular}{|c|c|c|c|c|c|c|c|c|c|c|c|c|c|}
\hline \multirow[b]{2}{*}{ Predictors } & \multirow[b]{2}{*}{$r$} & \multicolumn{4}{|c|}{ Model $1^{a}$} & \multicolumn{4}{|c|}{ Model $2^{b}$} & \multicolumn{4}{|c|}{ Model $3^{c}$} \\
\hline & & $b$ & S.E. & Wald & $\mathrm{p}$ & $\mathrm{b}$ & S.E. & Wald & $\mathrm{p}$ & $\mathrm{b}$ & S.E. & Wald & $\mathrm{p}$ \\
\hline \multicolumn{14}{|c|}{ High intention vs. no clear decision $(N=733)$} \\
\hline Attitude & $.73 * *$ & 1.86 & .18 & 101.67 & .000 & 1.63 & .21 & 61.09 & .000 & 1.38 & .25 & 30.78 & .000 \\
\hline Subjective Norm & $.37 * *$ & .19 & .12 & 2.59 & .11 & .11 & .13 & .78 & .38 & .18 & .15 & 1.45 & .23 \\
\hline $\begin{array}{l}\text { Perceived } \\
\text { Susceptibility }\end{array}$ & $.63^{* *}$ & .71 & .11 & 42.56 & .000 & .60 & .12 & 24.53 & .000 & .64 & .16 & 16.77 & .000 \\
\hline $\begin{array}{l}\text { Perceived } \\
\text { Severity }\end{array}$ & $.27^{* *}$ & -.15 & .14 & 1.09 & .30 & -.20 & .15 & 1.74 & .19 & .12 & .19 & .44 & .51 \\
\hline Autonomy & .03 & .01 & .114 & .01 & .92 & -.05 & .12 & .16 & .69 & .10 & .14 & .56 & .46 \\
\hline Capacity & $.31 * *$ & .25 & .16 & 2.33 & .13 & .15 & .18 & .70 & .40 & .38 & .22 & 2.92 & .09 \\
\hline Omission bias & $-.52 * *$ & & & & & -.16 & .11 & 2.22 & .14 & -.11 & .14 & .57 & .45 \\
\hline $\begin{array}{l}\text { Naturalistic } \\
\text { beliefs }\end{array}$ & $-.57 * *$ & & & & & -.39 & .13 & 8.95 & .003 & -.37 & .16 & 5.16 & .02 \\
\hline Disbelief science & $-.47 * *$ & & & & & -.14 & .12 & 1.24 & .27 & -.17 & .15 & 1.30 & .26 \\
\hline $\begin{array}{l}\text { Disbelief } \\
\text { relevance }\end{array}$ & $-.52 * *$ & & & & & .15 & .13 & 1.30 & .25 & .18 & .15 & 1.36 & .24 \\
\hline $\begin{array}{l}\text { Prevention } \\
\text { beliefs }\end{array}$ & $.18^{* *}$ & & & & & -.03 & .11 & .08 & .78 & -.08 & .13 & .40 & .53 \\
\hline Moral norm & $.36 * *$ & & & & & -.01 & .11 & .01 & .92 & .07 & .14 & .23 & .64 \\
\hline Responsibility & $.55^{* *}$ & & & & & .28 & .12 & 5.45 & .02 & -.10 & .16 & .40 & .53 \\
\hline Self-protection & $-.36 * *$ & & & & & -.18 & .10 & 3.53 & .06 & -.25 & .11 & 5.07 & .02 \\
\hline Management & $.09 *$ & & & & & -.20 & .12 & 2.49 & .11 & -.39 & .16 & 6.22 & .01 \\
\hline Flu last season & $-.07 *$ & & & & & & & & & .04 & .17 & .07 & .80 \\
\hline $\begin{array}{l}\text { Vaccination } \\
\text { frequency }\end{array}$ & $.70 * *$ & & & & & & & & & 1.01 & .15 & 46.09 & .000 \\
\hline Age group & $.19 * *$ & & & & & & & & & -.08 & .15 & .27 & .60 \\
\hline Gender & -.07 & & & & & & & & & -.27 & .37 & .53 & .47 \\
\hline Physician & $.08^{*}$ & & & & & & & & & .21 & .59 & .13 & .72 \\
\hline Nursing staff & $-.08 *$ & & & & & & & & & .22 & .46 & .22 & .64 \\
\hline $\begin{array}{l}\text { Other HCWs, p. } \\
\text { contact }\end{array}$ & .03 & & & & & & & & & .35 & .55 & .39 & .53 \\
\hline $\begin{array}{l}\text { Non-HCWs, no } \\
\text { p. contact }\end{array}$ & . & & & & & & & & & . & . & . & . \\
\hline The Netherlands & $-.21 * *$ & & & & & & & & & .61 & .50 & 1.46 & .23 \\
\hline Belgium & $.18^{* *}$ & & & & & & & & & .59 & .51 & 1.32 & .25 \\
\hline Germany & . & & & & & & & & & . & . & . & . \\
\hline Pseudo $R^{2}$ & & .73 & & & & .77 & & & & .82 & & & \\
\hline $\begin{array}{l}\text { Classification } \\
\text { accuracy (\%) }\end{array}$ & & 75.1 & & & & 77.1 & & & & 78.8 & & & \\
\hline
\end{tabular}

a Model 1 refers to a multinominal logistic regression with only the social cognitive variables as predictors.

b Model 2 refers to multinominal logistic regression with the social cognitive variables and additional beliefs as predictors.

c Model 3 refers to multinominal logistic regression with the social cognitive variables, additional beliefs, and demographics and past behavior as predictors.

$* p<.05$, two-tailed; ** $p<.01$, two-tailed. 


\section{DISCUSSION}

The results indicate that there are differences between HCWs from neighboring countries. Belgian HCWs scored overall highest on social cognitive variables and additional beliefs that contribute positively towards getting vaccinated. They also had the highest vaccination uptake (53\%) and the highest number of past vaccinations. In contrast, Dutch HCWs scored overall highest on social cognitive variables and additional beliefs that contribute negatively to the intention of getting vaccinated and showed the lowest vaccination uptake among the three countries (28.2\%). German HCWs were more similar to the Belgian than the Dutch HCWs with respect to social cognitive variables and additional beliefs. The vaccination uptake of German HCWs was $36.4 \%$ and a large proportion consisted of physicians, which are known to be more favorable towards influenza vaccination than nursing staff (Aguilar-Díaz et al., 2011; Zhang, While, \& Norman, 2012).

Results further suggested that different social cognitive variables, additional beliefs, past experiences and demographics are influential in the explanation of whether HCWs have no intention, are unsure, or have a high intention to get vaccinated against influenza. Attitude was the strongest predictor and correlate of intention and in turn correlated moderately to strongly with the additional beliefs included in the analyses. The second strongest predictor of intention was the past behavior of participants, or how often they had been vaccinated in the past. Even though we cannot change past behaviors, they are important to detect, because they shape the perceptions of people and seem to affect the intention to perform behaviors. In previous studies, strong perceived and moral norms were found to promote influenza vaccination (Hakim, Gaur, \& McCullers, 2011; Van den Dool et al., 2008). However, this study suggests that the perception of colleagues as being opposed to vaccination and not getting vaccinated, as well as not feeling morally responsible to get vaccinated to protect others, lowers the motivation to get vaccinated, while the opposite does not lead to a high intention. HCWs who reported that self-protection was not their main reason for vaccination and those that were more satisfied with the hospital management, were more likely to report a high intention towards vaccination. Also, in line with van den Dool et al. (2008), high perceived susceptibility was found to be a predictor of vaccination intention.

Some factors were identified that had not been associated with the intention to get vaccinated before: having an omission bias or preferring to not get vaccinated if one thinks that vaccination could cause illness. However, the omission bias is related to other well-documented reasons for non-immunization: the fear of side-effects, fear of long-term illness, and that the vaccine could cause influenza (Cohen \& Casken, 2012). Naturalistic beliefs were common among HCWs in our earlier qualitative study. The absence of naturalistic beliefs, respectively believing that vaccination is more fa- 


\section{Chapter 5}

vorable for one's health than undergoing illness, were promoting factors for the intention to get vaccinated.

Across the three samples we were able to explain a substantial amount of variance in intention to obtain influenza vaccination. However, some limitations are worth noting. Firstly, some of the concepts were only measured with one item, to reduce the length of the survey for an already time-pressured group of participants. This could have led to lower measurement specificity. Secondly, there could be a response bias. The response rate of hospitals willing to participate was low (25.6\%) and could have led to an overrepresentation of hospitals with an already well-established vaccination program, respectively a high vaccination coverage rate. Thirdly, we did not consider influenza vaccination in the presence of possible comorbidities of participants. It should be noted that their reasons for getting vaccinated against influenza could be related to their condition, rather than their occupation. Finally, for anonymity-reasons, participants were not linked to their hospital. Therefore, we could not make a difference between HCWs working in smaller, peripheral hospitals and those working in university hospitals, and were also not able to control for cluster effects in the hospital level.

\section{CONCLUSIONS}

In conclusion, this study showed that even though similar reasons for and against influenza vaccination were found for HCWs worldwide, there are differences in enabling and inhibiting social cognitive variables and additional beliefs that influence the intention to get vaccinated of HCWs from neighboring countries. Intervention development might therefore benefit from a more country-specific approach. Moreover, it can be beneficial for interventions with the aim of improving influenza vaccination coverage rates to focus on different social cognitive variables and additional beliefs depending on whether HCWs have the intention to get vaccinated against influenza or not. Our findings suggest that different factors are influential for immunizers and nonimmunizers. 


\section{CHAPTER 6}

The intention to get vaccinated against influenza and actual vaccination uptake of Dutch health care workers

Published as:

Lehmann, B. A., Ruiter, R. A. C., Chapman, G., \& Kok, G. (2014). The intention to get vaccinated against influenza and actual vaccination uptake of Dutch healthcare personnel. Vaccine, 32, 6986-6991. 


\section{Chapter 6}

\section{ABSTRACT}

Health Authorities recommend annual vaccination of health care workers (HCWs) against influenza to protect vulnerable patients. Nevertheless, vaccination rates have been low among European HCWs. Here we report on a longitudinal survey study to identify social cognitive predictors of the motivation to obtain influenza vaccination, and to test whether intention is a good predictor of actual vaccination behavior. Dutch HCWs $(N=1370)$ were invited to participate in a survey (baseline). To link intention to behavior, participants who completed the first survey $(N=556)$ were sent a second survey after vaccinations were offered (follow-up). Multinominal regression analysis showed that HCWs with a positive attitude and a higher frequency of past vaccinations were more likely to have a high intention to get vaccinated. A negative attitude, high feelings of autonomy in the decision whether to get vaccinated, a preference of inaction over vaccination, a lesser sense of personal responsibility, and high self-protection motives increased the probability of no intention to get vaccinated. Social cognitive predictors were identified that explain the intention to get vaccinated against influenza of HCWs, which in turn proved to be a good predictor of behavior. Future interventions should focus on these variables to increase vaccination coverage rates. 


\section{INTRODUCTION}

Influenza vaccination of health care workers (HCWs) reduces all-cause morbidity and mortality especially of those at high risk for influenza complications: young children, people above the age of 65 and high-risk patients (Ahmed, Lindley, Allred, Weinbaum, \& Grohskopf, 2014; Amodio et al., 2014; Mertz et al., 2013; WHO, 2005). Focusing on Europe, all HCWs are advised by Health Authorities to get vaccinated against influenza annually (CDC, 2011; WHO, 2009). Unfortunately, with vaccination coverage rates ranging from 6.4-26.3\% among European HCWs (Blank, Schwenkglenks, \& Szucs, 2009; Endrich, Blank, \& Szucs, 2009), the recommendations have not had their intended impact, and recent intervention programs developed to increase vaccination rates show at most small effects (Doratotaj, Macknin, \& Worley, 2008; Llupià et al., 2010; Looijmans-van den Akker et al., 2009; Looijmans-van den Akker et al., 2010; Looijmansvan den Akker et al., 2011).

In order to identify the social cognitive variables that predict influenza vaccination uptake by HCWs, a detailed analysis is needed. As suggested by Kok et al. (2011), systematic approaches (i.e. Intervention Mapping) have the potential to eventually lead to the successful development and implementation of programs to increase vaccination coverage rates among HCWs. We therefore developed an online survey instrument, which assessed a combination of social cognitive variables from the Reasoned Action Approach (RAA) (Fishbein \& Ajzen, 2010), and previous research (Lehmann, Ruiter, Wicker, van Dam, \& Kok, 2014). The purpose of the present study was to replicate results of one of our previous cross-sectional studies that had shown that the utilized social cognitive variables contribute largely to the explanation of HCWs' motivation to get vaccinated against influenza (Lehmann, Ruiter, van Dam, Wicker, \& Kok, 2015). However, this time we additionally conducted a follow-up survey to test whether the intention to get vaccinated, as well as the measured social cognitive variables, are good predictors of the actual vaccination behavior of HCWs.

The RAA is a social cognition model that specifies potentially modifiable antecedents of health behaviors (Fishbein \& Ajzen, 2010). The basic assumption of this model is that the motivation to perform a certain behavior is reflected in people's intention, which is determined by attitude, perceived norms, and perceived behavioral control. We further included measures of risk perception, which includes the constructs of perceived susceptibility to experience negative consequences if one does not perform the behavior under consideration and the perceived severity of those consequences. Moreover, the survey includes questions covering possible motivating factors for vaccination uptake (i.e. feelings of personal responsibility to protect others, selfprotection motives), and inhibiting factors for vaccination uptake (i.e. the disbelief in the scientific evidence of the effectiveness of influenza vaccination and its relevance) that have been described in previous research (Aguilar-Díaz, Jiménez-Corona, \& Poncede-León-Rosales, 2011; Cohen \& Casken, 2012; Hofmann, Ferracin, Marsh, \& Dumas, 
2006; Hollmeyer, Hayden, Poland, \& Buchholz, 2009; Looijmans-van den Akker et al., 2009; Van den Dool et al., 2008; Wicker, Rabenau, Doerr, \& Allwinn, 2009). Next to these concepts, measures of three additional beliefs were included that had been identified in a qualitative study we recently conducted (Lehmann et al., 2014). Some people had indicated that they favor risking an illness instead of performing a behavior that might prevent illness such as vaccination, when the performance of the behavior itself is believed to entail risk. We called this phenomenon omission bias, taking over Asch and colleagues' definition of the preference of inaction over action, even though inaction is more likely to result in a harmful outcome (Asch et al., 1994). Another commonly stated reason for non-immunization was the belief that vaccination weakens the natural immune system, which will be referred to as naturalistic beliefs (da Costa DiBonaventura \& Chapman, 2008). Finally, prevention beliefs constitute the opinion that other means of prevention (i.e. regular hand disinfection, staying at home when ill) are more effective in preventing influenza than vaccination (Bridges, Kuehnert, \& Hall, 2003).

The aim of this longitudinal study was to test with a survey whether the intention to get vaccinated, as well as the measured social cognitive variables, are good predictors of the actual vaccination behavior of HCWs. The social cognitive variables that will be identified to predict actual vaccination uptake can serve as reference points for the systematic development of a program to increase influenza vaccination uptake of HCWs.

\section{METHODS}

\section{Participants and procedure}

Dutch HCWs belonging to an online panel $(N=1370)$ were invited in the last week of September 2013 to participate in a longitudinal survey about the factors that influence the decision to get vaccinated against influenza (baseline). HCWs in the Netherlands commonly get offered influenza vaccination between October and November. Participants who got vaccinated before the last week of September were excluded from the sample $(N=23)$, as were HCWs that indicated that they did not have direct patient contact $(N=199)$. In total, 556 participants were included in the baseline measure (response rate $40.6 \%$ ). To link intention to actual vaccination behavior, participants who completed the first questionnaire were sent a second questionnaire in the last week of November 2013 (follow-up). The follow-up survey was completed by 458 (82\%) participants. 


\section{The questionnaires}

The first online questionnaire consisted of 42 questions targeting social cognitive variables and additional beliefs about annual influenza vaccination, past behavior, and socio-demographics. Variables were measured on 7-point Likert scales ranging from 1 $=$ totally disagree to 7 = totally agree, unless otherwise indicated. Items measuring the same underlying theoretical construct were averaged into one single construct when internal consistency was sufficient (Cronbach's alpha $\alpha>.60$ or Pearson correlation coefficient $r>.40$ ). Table 6.1 provides an overview of the constructs and their internal consistency.

Table 6.1 Overview of constructs measured by the online survey

\begin{tabular}{|c|c|c|c|}
\hline Variable & $\begin{array}{l}\text { Number } \\
\text { of items }\end{array}$ & Reliability & Example questions \\
\hline Intention & 2 & $r=.92$ & I intend to get vaccinated against influenza annually. \\
\hline Attitude & 6 & $\alpha=.90$ & $\begin{array}{l}\text { Getting vaccinated against influenza annually is: very } \\
\text { good - very bad; comforting - frightening. }\end{array}$ \\
\hline Subjective Norm & 4 & $\alpha=.77$ & $\begin{array}{l}\text { Most of my colleagues get vaccinated against } \\
\text { influenza annually. }\end{array}$ \\
\hline Perceived susceptibility & 2 & $r=.40$ & $\begin{array}{l}\text { I am healthy, therefore I don't need to get vaccinated } \\
\text { against influenza annually. }\end{array}$ \\
\hline Perceived severity & 2 & $r=.48$ & $\begin{array}{l}\text { Influenza is a serious infection that can lead to } \\
\text { complications. }\end{array}$ \\
\hline Autonomy & 1 & n.a. & $\begin{array}{l}\text { Getting vaccinated against influenza annually is } \\
\text { completely up to me. }\end{array}$ \\
\hline Capacity & 1 & n.a. & $\begin{array}{l}\text { I am confident that I could get vaccinated against } \\
\text { influenza annually (if I want to). }\end{array}$ \\
\hline Omission bias & 1 & n.a. & $\begin{array}{l}\text { I prefer to get influenza, instead of getting vaccinated } \\
\text { against influenza. }\end{array}$ \\
\hline Naturalistic beliefs & 3 & $\alpha=.87$ & $\begin{array}{l}\text { I think that it is better to undergo influenza, then to } \\
\text { get vaccinated against influenza annually. }\end{array}$ \\
\hline Disbelief science & 2 & $r=.70$ & $\begin{array}{l}\text { As far as I know, there is insufficient scientific } \\
\text { evidence that influenza vaccination is effective in } \\
\text { preventing influenza. }\end{array}$ \\
\hline Disbelief relevance & 3 & $\alpha=.81$ & $\begin{array}{l}\text { I think that the relevance of the annual influenza } \\
\text { vaccination is overestimated. }\end{array}$ \\
\hline Prevention beliefs & 3 & $\alpha=.65$ & $\begin{array}{l}\text { By staying at home when I am ill, I can sufficiently } \\
\text { protect patients from getting influenza. }\end{array}$ \\
\hline Personal responsibility & 4 & $\alpha=.72$ & $\begin{array}{l}\text { I think it is part of the responsibilities as a HCW to get } \\
\text { vaccinated against influenza annually. }\end{array}$ \\
\hline Self-protection & 1 & n.a. & $\begin{array}{l}\text { If I would get vaccinated against influenza annually, I } \\
\text { would do it to protect myself. }\end{array}$ \\
\hline
\end{tabular}


In addition, past behavior was measured with two questions ('In past years I got vaccinated against influenza, when it was offered to me: 1 = always; 7 = never.'; 'Did you get vaccinated against influenza this year (season 2012/ 2013)? yes/no.'). Past experience with influenza was measured with two questions ('How often did you have influenza in the past? 1 = never; 7 = more than 10 times.'; 'Did you have influenza last winter? no/yes, once/yes, more than once.'). These items measured own experiences of influenza-like illness (ILI) instead of laboratory confirmed influenza. Demographic measures assessed profession (physician/nursing staff/other HCWs), gender and age.

The follow-up questionnaire consisted of five questions. Behavior was measured with one question ('Did you get vaccinated against influenza in the past three months? yes/ no'). Participants who indicated that they got vaccinated against influenza were asked about the vaccination location and experiences with the vaccination ('Where did you get vaccinated against influenza? At work/ at my general practitioner/ other, namely'; How would you describe your vaccination experience? 1=very good; 7=very bad, 1=very pleasant; $7=$ very unpleasant, $1=$ very painful; $7=$ not at all painful; Did you experience a reaction or side-effects from the vaccine? Specify.'). Participants who indicated that they did not get vaccinated were asked to specify their reasons for nonimmunization ('Specify shortly why you did not get vaccinated against influenza.').

\section{Data analysis}

SPSS 20.0 was used to analyze the data. Following a descriptive analysis of the sample (frequencies), univariate associations between intention and social cognitive variables were analyzed with Pearson correlation coefficients. Intention was shown to be distributed U-shaped and to best be classified into three groups; no intention to get vaccinated against influenza $(0=1.0-2.0)$, not having made a clear decision about vaccination ( $1=2.5-5.5)$, and a high intention to get vaccinated $(2=6.0-7.0)$. Therefore, multinominal logistic regression was used to show the effect of the independent variables on the probability of (1) having no intention to get vaccinated vs. not having made a clear decision and (2) having a high intention to get vaccinated vs. not having made a clear decision. A logistic regression that included only HCWs who participated in the follow-up examined the link between intention and the independent variables used to predict intention at baseline to actual vaccination behavior at follow-up.

\section{RESULTS}

\section{Response and descriptive statistics}

At baseline, the study sample consisted of 556 participants (see Table 6.2). Of the total sample, 86 were male (15\%) and 470 were female (85\%). Participants had a mean age of 39.9 years (range 19 to 67). The sample consisted of 173 participants working in 
hospital settings (31\%), 94 were physicians (17\%), 139 were nursing staff $(25 \%)$, and 323 (58\%) indicated being other HCWs (e.g., paramedics, physiotherapists, dieticians). In the Netherlands, there are 333.939 registered care givers, of which $23 \%$ are physicians, $54 \%$ are nursing staff, and $23 \%$ are other HCWs. Of the respondents, 458 (82\%) participated in the follow-up and were included in the analysis to assess the extent to which intention predicts behavior.

Table 6.2 Demographics and HCW characteristics

\begin{tabular}{|c|c|c|c|}
\hline & Total $(\mathrm{N}=556)$ & $\begin{array}{l}\text { Vaccinated } \\
(\mathrm{N}=90,19.7 \%)\end{array}$ & $\begin{array}{l}\text { Not vaccinated } \\
(\mathrm{N}=368,80.3 \%)\end{array}$ \\
\hline \multicolumn{4}{|l|}{ Gender } \\
\hline Male & $86(15.5)$ & 17 (18.9) & $57(15.5)$ \\
\hline Female & $470(84.5)$ & $73(81.1)$ & $311(84.5)$ \\
\hline Age (mean, SD) & $39.9(11.6)$ & $44.2(12.9)$ & $38.8(11.1)$ \\
\hline \multicolumn{4}{|l|}{ Occupation } \\
\hline Hospital HCWs & $173(31.1)$ & $37(41.1)$ & $102(27.7)$ \\
\hline Physician & $94(16.9)$ & $19(21.1)$ & $56(15.2)$ \\
\hline Nursing staff & $139(25.0)$ & $25(27.8)$ & $92(25)$ \\
\hline Other HCWs & $323(58.1)$ & $46(51.1)$ & $220(59.8)$ \\
\hline ILI in season 2012-2013 & $113(20.4)$ & 17 (18.9) & $65(17.6)$ \\
\hline
\end{tabular}

Data are reported as number of participants (\%).

\section{Social-cognitive variables of HCWs' vaccination intention}

Table 6.3 shows that all social cognitive variables and additional beliefs were significantly correlated with intention. A small effect is $r=.10-.23$, a moderate effect $r=.24$ .36 and a large effect is $r \geq .37$ (Cohen, Cohen, West, \& Aiken, 2003).

We found strong positive univariate associations with intention for attitude, perceived norm, perceived susceptibility, personal responsibility, and past vaccination frequency. Strong negative associations with intention were found for having an omission bias, holding naturalistic views, for the disbelief in scientific evidence that influenza vaccination is effective, and the disbelief in the relevance of the flu shot. Results of the multinominal logistic regression are shown in Table 6.4. 


\section{Chapter 6}

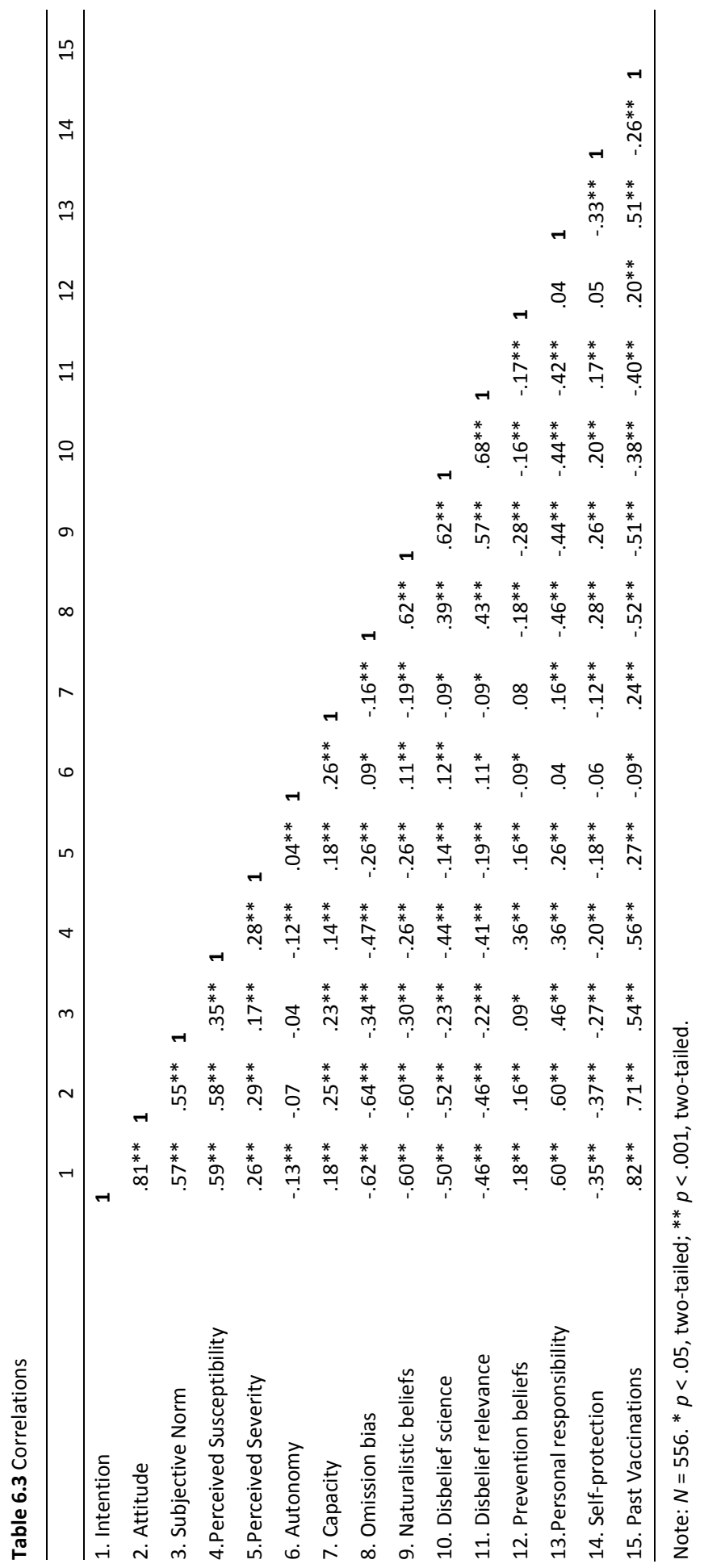


Table 6.4 Multinominal logistic regression

\begin{tabular}{|c|c|c|c|c|c|}
\hline Predictors & $\mathbf{r}$ & b & S.E. & Wald & $\mathbf{p}$ \\
\hline \multicolumn{6}{|c|}{ No intention vs. no clear decision ( 1 vs. $0 ; N=441)$} \\
\hline Attitude & $-.53^{* *}$ & -1.35 & .28 & 23.32 & $<.001$ \\
\hline Subjective Norm & $-.34^{* *}$ & -.21 & .15 & 1.85 & .17 \\
\hline Perceived Susceptibility & $-.30^{* *}$ & -.12 & .14 & .81 & .37 \\
\hline Perceived Severity & $-.13^{* *}$ & .03 & .14 & .06 & .82 \\
\hline Autonomy & $.23^{* *}$ & .27 & .12 & 4.98 & .03 \\
\hline Capacity & -.00 & .13 & .08 & 2.40 & .12 \\
\hline Omission bias & $.41^{* *}$ & .24 & .10 & 5.53 & .02 \\
\hline Naturalistic beliefs & $.37^{* *}$ & .09 & .15 & .35 & .56 \\
\hline Disbelief science & $.31^{* *}$ & .18 & .17 & 1.14 & .29 \\
\hline Disbelief relevance & $.31^{* *}$ & .27 & .18 & 2.32 & .13 \\
\hline Prevention beliefs & $-.10^{*}$ & .03 & .14 & .04 & .84 \\
\hline Personal responsibility & $-.39 * *$ & -.41 & .16 & 6.79 & .01 \\
\hline Self-protection & $.20^{* *}$ & .22 & .09 & 5.67 & .02 \\
\hline Past vaccination frequency & $-.48^{* *}$ & -.44 & .10 & 19.54 & $<.001$ \\
\hline \multicolumn{6}{|c|}{ High intention vs. no clear decision ( 1 vs. $0 ; \mathrm{N}=274$ ) } \\
\hline Attitude & $.69^{* *}$ & 1.31 & .34 & 14.87 & $<.001$ \\
\hline Subjective Norm & $.37^{* *}$ & .13 & .23 & .33 & .57 \\
\hline Perceived Susceptibility & $.51^{* *}$ & .27 & .21 & 1.64 & .20 \\
\hline Perceived Severity & $.20^{* *}$ & -.14 & .24 & .32 & .57 \\
\hline Autonomy & $.14^{*}$ & .18 & .20 & .82 & .36 \\
\hline Capacity & $.26^{* *}$ & -.11 & .16 & .48 & .50 \\
\hline Omission bias & $-.46^{* *}$ & -.26 & .19 & 1.83 & .18 \\
\hline Naturalistic beliefs & $-.42^{* *}$ & -.18 & .25 & .42 & .52 \\
\hline Disbelief science & $.34^{* *}$ & -.40 & .22 & 3.23 & .07 \\
\hline Disbelief relevance & $-.30^{* *}$ & .16 & .25 & .42 & .52 \\
\hline Prevention beliefs & .12 & -.29 & .20 & 2.04 & .15 \\
\hline Personal responsibility & $.46^{* *}$ & -.03 & .27 & .01 & .91 \\
\hline Self-protection & $-.24^{* *}$ & -.06 & .14 & .21 & .65 \\
\hline Past vaccination frequency & $.70^{* *}$ & .79 & .15 & 29.91 & $<.001$ \\
\hline Pseudo $R^{2}$ & & .80 & & & \\
\hline Classification accuracy (\%) & & 82 & & & \\
\hline
\end{tabular}

$* p<.05$, two-tailed; $* * p<.01$, two-tailed.

HCWs were more likely to have no intention to get vaccinated vs. not having made a clear decision when they reported a negative attitude towards influenza vaccination and high feelings of autonomy, when they showed a stronger omission bias, a lesser sense of personal responsibility to protect patients by getting vaccinated, when they reported high self-protection motives, and lower frequency of influenza vaccinations in the past. 
When comparing having a high intention vs. not having made a clear decision, we found that HCWs with a positive attitude towards influenza vaccination and a higher frequency of influenza vaccinations in the past were more likely to have a high intention vs. not having made a clear decision. No other significant unique contributions to the prediction of having a high intention were found. The variables in the regression model explained $80 \%$ of the variance in intention (Pseudo $R^{2}=.80$ ), with a classification accuracy of $82 \%$.

\section{Additional analyses}

In an exploratory manner we excluded the most influential variable, attitude, from the multinominal analysis, because we hypothesized that it might overrule the (indirect) influence of other variables on intention. Only one additional significant predictor appeared in this analysis: higher sense of personal responsibility significantly predicts a high intention to get vaccinated as opposed to an unclear decision when attitude is excluded.

We next tested whether attitude mediates the relationship between personal responsibility and high intention vs. an unclear decision. To test for mediation, we used the SPSS macros that Preacher and Hayes (2004) provide for a binary logistic regression with bootstrapping technique. The bias corrected and accelerated (BCa) confidence intervals were set at 0.95 with 5000 resamples. The mediation analysis revealed that there is a meaningful indirect effect of attitude on the relationship between personal responsibility and intention ( $b=1.29, \mathrm{BCa} 95 \% \mathrm{Cl}[.874 ; 1.856])$, only for participants in the categories high intention vs. no clear decision $(N=274)$. The fact that zero falls outside this interval indicates a significant mediation effect. For the regression coefficients for the relationship between personal responsibility and intention (high/ unsure) as mediated by attitude, see Figure 6.1.

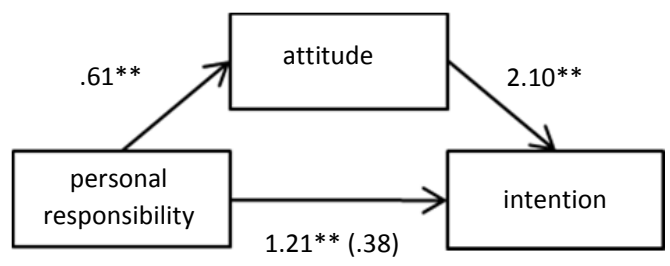

Figure 6.1 Regression coefficients for the relationship between personal responsibility and intention to get vaccinated (high/ unsure) as mediated by attitude. The path between personal responsibility and attitude is an OLS regression coefficient, while the other paths are logistic regression coefficients. The logistic regression coefficient between personal responsibility and intention, controlling for attitude, is in parentheses.

$* p<.05 ; * * p<.01$ 


\section{Social-cognitive variables of HCWs' vaccination behavior}

Table 6.5 shows that amongst the HCWs that got vaccinated against influenza, the majority had reported to have a high intention to get vaccinated at baseline $(N=68$, $73.9 \%)$. The percentage of participants that were vaccinated differed by intention, $\chi^{2}$ $(2, N=458)=224.42, p<.001$. Of the HCWs who participated in the follow-up survey $(N=458), 90(19.7 \%)$ got vaccinated against influenza.

Table 6.5 Crosstab of HCWs' intention to get vaccinated and their actual vaccination behavior

\begin{tabular}{lcclc}
\hline & No intention & No clear decision & High intention & Total \\
\hline Not vaccinated & $235(98.7)$ & $109(85.2)$ & $24(26.1)$ & $268(80.3)$ \\
Vaccinated & $3(1.3)$ & $19(14.8)$ & $68(73.9)$ & $90(19.7)$ \\
Total & $238(100)$ & $128(100)$ & $92(100)$ & $458(100)$ \\
\hline
\end{tabular}

Data are reported as number of participants (\%).

Intention was a significant predictor of vaccination behavior (OR=15.50, 95\% Cl: 9.2425.99). Intention to get vaccinated explained $58 \%$ of the variance in behavior (Nagelkerke $R^{2}=.58$ ). Attitude and past vaccination frequency explained an additional $6 \%$ in behavior (Nagelkerke $R^{2}=.64$ ).

Of those that got vaccinated $(N=90), 43(47.8 \%)$ indicated that they had gotten vaccinated at work and $47(52.2 \%)$ indicated receiving vaccination from their general practitioner. The three items measuring vaccination experience showed high internal consistency $(\alpha=.76)$ and were averaged into one construct. With an average score of $5.6(S D=1.3)$ on a 7-point scale, the vaccination experience can generally be described as positive. Reactions to or side-effects from the vaccine were reported by 33 participants who got vaccinated. The most common reported occurrence were a minor local reaction at the site of injection $(N=27)$, followed by general malaise $(N=4)$, flu-like symptoms $(N=3)$, and having a cold $(N=2)$. Headaches and influenza were each indicated once.

HCWs who did not get vaccinated ( $N=368 ; 80.4 \%$ ) were asked to specify their reasons for non-immunization. A low risk perception was indicated most often by HCWs ( $N=234,49.6 \%)$, followed by organizational issues ( $N=58,12.3 \%)$, such as time constraints, not being offered the vaccination, or absence. The disbelief in the effectiveness of the vaccine in protecting oneself or others was reported 45 times and fear of side-effects or illness from the vaccine was reported by 43 participants. Misconceptions including the belief that the vaccine weakens the immune system and the belief that pregnant women should not get vaccinated were reported by 36 of the participants. Some non-immunizers indicated feeling negative about getting something injected $(N=15)$. Few participants indicated medical reasons $(N=3)$, fear of needles $(N=$ 
1) and the advice of their general practitioner to not get vaccinated $(N=1)$ as reasons for non-immunization. Two participants indicated that they were still planning to get vaccinated.

\section{DISCUSSION}

This study shows that, relative to having no clear intention, different social cognitive variables predict high versus no intention to get vaccinated against influenza. In accordance with a previous study from our institute, the only factors shown to be indicative of both, having no intention and having a high intention to get vaccinated were attitude and past vaccination frequency. Attitude seems to be most influential for the prediction of intention and is also the strongest correlate of intention. Positive attitudes and previous vaccine receipt had been shown to be predictors of vaccination uptake in past research (Aguilar-Díaz et al., 2011; Hollmeyer et al., 2009; Van den Dool et al., 2008).

Previous research has shown that autonomy in the decision whether to get vaccinated against influenza is highly valued by HCWs (Hakim, Gaur, \& McCullers, 2011), and our study shows that, compared to those with no clear intention, HCWs with no intention to get vaccinated have high feelings of autonomy. Moreover, low feelings of personal responsibility to protect people in the environment and strong self-protection motives were associated with having no intention to get vaccinated. These findings are in contradiction with previous studies that had shown that self-protection is amongst the most often reported facilitating factors of influenza vaccination uptake (Hakim et al., 2011; Looijmans-van den Akker et al., 2009; Van den Dool et al., 2008). The efforts to improve vaccination uptake of HCWs are primarily motivated by the fact that vaccinating HCWs can reduce all-cause morbidity and mortality of vulnerable patients (Ahmed et al., 2014; Amodio et al., 2014; Mertz et al., 2013; WHO, 2005). Therefore, it is important that HCWs themselves feel personally responsible to protect their patients through vaccination. Although we found that low feelings of personal responsibility were associated with having no intention to vaccinate, relative to having no clear intention, surprisingly, we did not find an influence of personal responsibility on high intention to get vaccinated, which let us to investigate a possible mediation effect. Indeed, we found that feelings of personal responsibility did predict high intention, relative to unsure intention, but this effect was mediated by attitude. Our findings suggest that addressing feelings of responsibility might therefore be an important determinant to focus on in changing attitudes.

Furthermore, we replicated the finding that HCWs who prefer not to get vaccinated because of the fear that the vaccines might cause harm, are more likely to have no intention to get vaccinated. This omission bias had previously been shown to decrease 
the likelihood of accepting influenza vaccination (da Costa DiBonaventura \& Chapman, 2008).

Interestingly, there were many more unique predictors of no intention as opposed to being unsure than of high intention to get vaccinated. A possible explanation for this finding is that HCWs that have a high intention know exactly why they are willing to get vaccinated, while HCWs who have no intention to get vaccinated might not be able to justify their unwillingness and negative feelings as easily and might therefore be more susceptible to agree with the more negative end of the utilized items.

Of the HCWs who participated in the follow-up, fewer than $20 \%$ got vaccinated against influenza. The vaccination experience of immunizers was generally perceived as positive, with the most often reported side-effect being minor local pain. The reasons that were given by non-immunizers for not getting vaccinated are welldocumented inhibiting factors and misconceptions in the literature (Aguilar-Díaz et al., 2011; Cohen \& Casken, 2012; Hofmann et al., 2006; Hollmeyer et al., 2009; Van den Dool et al., 2008; Wicker et al., 2009). Almost half of the non-immunizers indicated not feeling at risk of getting infected with influenza. Moreover, organizational barriers, doubts about the effectiveness of the vaccine, and fear of adverse effects from the vaccine were reported. Misconceptions included the belief that the vaccine weakens the immune system and the belief that pregnancy is a contraindication for influenza vaccination. In 2013, the Global Advisory Committee on Vaccine Safety (GACVS) established by the WHO concluded that influenza vaccination of pregnant women is safe and beneficial for the mother and the unborn child (2013).

Because our study included a follow-up survey we were able to link intention with actual vaccination behavior. Intention was a good predictor of HCWs' vaccination behavior, exceeding the average explained variance of intention-behavior relationships as stated in a meta-analysis by Sheeran (2002). The majority of HCWs who had a high intention to get vaccinated actually did get vaccinated, but only $15 \%$ of the HCWs who indicated being unsure about vaccination got vaccinated. HCWs in the latter category might be a promising group to target in future intervention programs to increase vaccination uptake. They have the highest potential of eventually making a transition to the high intention group, when the right determinants are targeted.

The current study had some limitations. We reduced the survey length in an attempt to improve response rates among HCWs by measuring some constructs with only one item, which could have lowered measurement specificity. Another limitation of this study is a possible response bias. HCWs who completed the follow-up survey likely expected to be asked about their vaccination status. Consequently, vaccinators may be overrepresented in our sample due to self-selection. Moreover, nursing staff and HCWs working in hospitals are slightly underrepresented in our sample, which might reduce the representativeness of Dutch HCWs as a whole. Finally, because of anonymity and confidentiality reasons we did not collect detailed data about the dif- 


\section{Chapter 6}

ferent occupational groups and specifics about participants' patient contact. This information could have been helpful in further stratifying the findings.

\section{CONCLUSIONS}

In conclusion, this study replicated one of our previous studies by showing that different factors are influential for immunizers and non-immunizers. A number of the socialcognitive variables we investigated contribute largely to the explanation of HCW' motivation to get vaccinated against influenza, and intention was a strong predictor of actual vaccination behavior. We plan to use these determinants to develop a program to promote influenza vaccination in HCWs using the Intervention Mapping approach (Bartholomew, Parcel, Kok, Gottlieb, \& Fernandez, 2011). 


\section{CHAPTER 7}

\section{Changing the default to promote influenza vaccination among health care workers}

Submitted as:

Lehmann, B. A., Chapman, G. B., Franssen, F. M. E., Kok, G., \& Ruiter, R. A. C. Changing the default to promote influenza vaccination among health care workers. 


\section{ABSTRACT}

The prevention of health care acquired infections is an important objective for patient safety and infection control in all health care settings. Influenza vaccination uptake among health care workers (HCWs) is the most effective method to prevent transmission to patients, but vaccination coverage rates are low among HCWs. Several educational campaigns have been developed to increase the influenza vaccination coverage rates of HCWs, but showed only small effects. The aim of this study was to test the default strategy in promoting uptake among HCWs in a tertiary care center for patients with complex chronic organ failure. HCWs were randomly assigned to one of two conditions. In the opt-out condition, participants received an e-mail with a pre-scheduled appointment for influenza vaccination, which could be changed or canceled. In the opt-in condition, participants received an e-mail explaining that they had to schedule an appointment if they wanted to get vaccinated. The findings show no statistically detectable effect of condition on being vaccinated against influenza. However, the difference of $11.5 \%$ in vaccination uptake between the conditions is comparable with another study that had used this approach to promote vaccination. Moreover, HCWs in the opt-out condition were more likely to have an appointment for influenza vaccination, which in turn increased the probability of getting vaccinated. To change the default to promote influenza vaccination among HCWs might be an easy and costeffective alternative to the complex vaccination campaigns that have been proposed in recent years. 


\section{INTRODUCTION}

The prevention of health care acquired or nosocomial infections is an important objective for patient safety and infection control in all health care settings (WHO, 2002). Several studies reported on the incidence of influenza infections leading to nosocomial outbreaks with negative consequences for patients and the health care organization (Amodio et al., 2014; Bénet et al., 2012; Salgado, Farr, Hall, \& Hayden, 2002; Sartor et al., 2002; Voirin, Barret, Metzger, \& Vanhems, 2009). A review including 12 nosocomial outbreaks in health care settings reported an infection prevalence of up to $50 \%$ among patients on the epidemic ward (Salgado et al., 2002). Sartor and colleagues (2002) found that $41 \%$ of patients and $23 \%$ of health care workers (HCWs) contracted influenza on an internal medicine ward during an outbreak, which resulted in additional morbidity, as well as considerable interferences with and delay of health care services.

Nosocomial outbreaks are especially problematic for immunosuppressed patients, including those with underlying chronic diseases leading to increased morbidity, mortality and associated costs (Glezen, Greenberg, Atmar, Piedra, \& Couch, 2000; Gorse et al., 2006; Macesic, Kotsimbos, Kelly, \& Cheng, 2013). In particular, patients with chronic obstructive pulmonary disease (COPD) have been shown to suffer from a $15-50 \%$ acute exacerbation following a respiratory infection (Gorse et al., 2006). Patients get infected with influenza through relatives, other patients, or HCWs. It is estimated that $20 \%$ of HCWs get infected with influenza annually (Elder, O'Donnell, McCruden, Symington, \& Carman, 1996). Many of them continue working and thereby promote the spread of influenza (Weingarten, Riedinger, Burnes Bolton, Miles, \& Ault, 1989). Vaccination against influenza is the most effective method to prevent nosocomial transmission (CDC, 2010; Dolan, Iredale, Williams, \& Ameen, 2004), and studies showed that vaccination helps to reduce influenza-related diseases and mortality among patients with chronic lung diseases (Wongsurakiat et al., 2004). A Dutch study executed in University hospitals showed that an increase of $10.8 \%$ in the vaccination uptake of HCWs through means of a multi-faceted program resulted in approximately $6 \%$ fewer patients with nosocomial influenza and/or pneumonia compared with control hospitals (Riphagen-Dalhuisen et al., 2013). In addition, studies clearly indicated that vaccinating HCWs is cost-effective (Burls et al., 2006; Nichol et al., 1995; Sartor et al., 2002).

Despite all evidence for the effectiveness of vaccination in the prevention of nosocomial infections, vaccination coverage rates among European HCWs are low. A study by Blank, Schwenkglenks, and Szucs (2009) in eleven European countries reported vaccination rates of between 6.4 and $26.3 \%$ among HCWs. Attitude is an important determinant predicting $\mathrm{HCWs}^{\prime}$ intention to get vaccinated against influenza (Lehmann, Ruiter, Chapman, \& Kok, 2014; Lehmann, Ruiter, van Dam, Wicker, \& Kok, 2015). This implies an educational strategy to persuade HCWs to get vaccinated. However, several educational campaigns have been developed to increase the influenza vaccination coverage rates of HCWs (Doratotaj, Macknin, \& Worley, 2008; Llupià et al., 2010; 
Looijmans-van den Akker et al., 2011; Riphagen-Dalhuisen, 2013), but showed only small effects. Consequently, there seems to be a need for a radically different approach to change vaccination behavior.

An approach that has shown to be effective in influencing behavior is nudging (Li \& Chapman, 2013). Nudges are small and simple changes in the environment that push decision makers in the right direction without restricting their choice autonomy. One such nudge that has shown to be able to promote health behaviors is the default effect (Goldstein, Johnson, Herrmann, \& Heitmann, 2008; Li \& Chapman, 2013). Decision makers show the tendency of sticking with a default option, the option that comes into effect if the decision maker does not actively decide against it. A study by Chapman, Li, Colby, and Yoon (2010) manipulated the default by sending e-mail appointments for annual influenza vaccination to University staff. Employees in the opt-out condition had an appointment by default and had to actively cancel it if they did not want to have an appointment (or they could ignore the appointment, which most did). Employees in the opt-in condition did not have an appointment and had to actively make an appointment if they wanted to have an appointment for vaccination (or they could be vaccinated as walk-ins). A $12 \%$ absolute increase in vaccination rate was found in favor of the opt-out condition. In addition, it was found that appointment status mediated the relationship between condition and getting vaccinated.

Because HCWs are an important source of nosocomial infections in vulnerable patient groups, and previous educational interventions have failed or only reached small effects, this study tested the use of the default strategy to increase the influenza vaccination uptake of HCWs in a Dutch expert center for patients with chronic organ failure using a randomized experimental design.

\section{METHODS}

\section{Setting, participants, design and procedure}

$\mathrm{CIRO}+$ is a center of expertise for the diagnosis and treatment of patients with complex chronic organ failure, in particular obstructive pulmonary diseases (i.a. COPD and asthma) and chronic heart failure. It is located in the south of the Netherlands. The center employs 122 people, including (chest) physicians, nursing staff, occupational therapists, physiotherapists, laboratory workers, biomechanical engineers, dieticians, and researchers. Most employees have patient contact.

In the beginning of October 2014, CIRO+ employees were invited to attend a presentation, outlining the available evidence regarding the effectiveness of influenza vaccination in protecting patients, during one of their regular educational seminars. In mid-October, all 122 employees at CIRO+ were randomly assigned to one of two conditions in a one-factorial between-subjects design (email invitation: opt-in vs. opt-out). 
Those in the opt-out condition received an e-mail from the responsible chest physician explaining that they had been scheduled for the annual influenza vaccination, with the day, time, and location provided. Vaccinations free of charge were given on two different days of the week. Hyperlinks in the e-mail allowed participants to change or cancel the appointment day and/or time. For those in the opt-in condition, the e-mail explained that there were two days on which free influenza vaccinations were available and they had to schedule an appointment with the chest physician if they wanted to get vaccinated. In the week of the vaccinations, all opt-out participants that had changed or did not cancel their appointment were sent a reminder e-mail. Opt-in participants were not sent a reminder.

\section{Data analysis}

Pearson Chi-Square analysis was conducted with SPSS 21.0 to test for a difference in influenza vaccination uptake between the opt-in and the opt-out condition. Mplus 7 was used to test for mediation of appointment status. The bias corrected and accelerated $(\mathrm{BCa})$ confidence intervals were set at .95 with 5000 resamples.

\section{RESULTS}

The study sample consisted of $122 \mathrm{CIRO}+$ employees, of which 97 (79.5\%) were female. Of the 61 participants that were randomly assigned to the opt-in condition, 12 scheduled an appointment, of which 8 got vaccinated, while 49 participants did not make an appointment, of which 2 got vaccinated. In the opt-out condition, 37 of the 61 participants cancelled their appointment. Of the 24 participants that did not cancel their appointment, 19 retained their original appointment of whom 12 got vaccinated and 7 did not. The appointment was changed to a different time and/or day by 5 participants; all 5 received the vaccination (see Table 7.1).

Table 7.1 Overview of HCWs' behavior in the two conditions and vaccination uptake

\begin{tabular}{lll}
\hline & Opt-in & Opt-out \\
\hline Assigned & 61 & 61 \\
Appointment & 12 & 24 (5 rescheduled) \\
Vaccinated & 10 (2 without appointment) & 17 \\
$\%$ & $16.4 \%$ & $27.9 \%$ \\
\hline
\end{tabular}

In the opt-in condition, 10 of 61 participants (16.4\%) were vaccinated against influenza, compared with 17 of 61 participants (27.9\%) in the opt-out condition, an $11.5 \%$ absolute difference $[95 \% \mathrm{Cl}, 3.3 \%-25.8 \%]$. Despite the non-significance of this differ- 
ence $\left(\chi^{2}(1, N=122)=2.33, p=.13\right)$, mediation analysis revealed that there is a meaningful indirect effect of appointment status (canceled vs. made/ kept) on the relationship between condition (opt-in vs. opt-out) and flu shot (yes vs. no) ( $b=.553$, BCa $95 \%$ $\mathrm{Cl}$ [.107;1.043]; see Figure 7.1). In the opt-in condition, 12 of the 61 staff members had an appointment, compared with 24 of the 61 staff members in the opt-out condition. Of the 36 staff members with an appointment, 25 got vaccinated, while only 2 of the 86 staff members without an appointment got vaccinated. The fact that zero falls outside the bootstrapped interval of the total effect indicates a significant mediation of the effect of opt-out vs. opt-in on vaccination rate through appointment status.

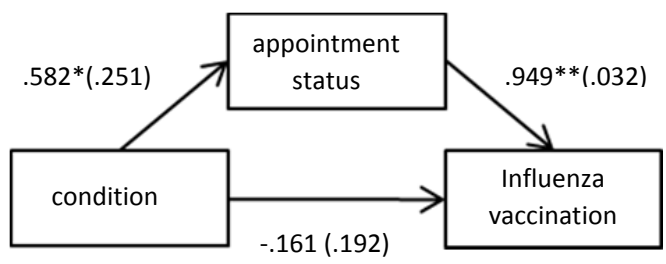

Figure 7.1 Logistic regression coefficients (S.E.) for the relationship between condition (opt-out vs. opt-in) and influenza vaccination (yes vs. no) as mediated by appointment status (yes vs. no).

$* p<.05 ; * * p<.01$

\section{DISCUSSION}

This study tested whether a default manipulation increases the influenza vaccination uptake rate among HCWs. We did not find a significant increase of the likelihood of the opt-out condition on the probability to get vaccinated. However, the difference of $11.5 \%$ in the influenza vaccination uptake rate between HCWs in the opt-out and in the opt-in condition is comparable with the difference reported by Chapman and colleagues (2010), who did report a significant effect of condition on vaccination uptake. Following the study of Chapman and colleagues (2010), we hypothesized that there might be an indirect effect of appointment status on this relationship. Contrary to what Baron and Kenny originally proposed (1986), others do suggest that there can be mediation in the absence of an effect of the independent variable on the dependent variable, as long as there is a significant relationship between the independent variable and the mediator, as well as a significant relationship between the mediator and the dependent variable (MacKinnon, Lockwood, Hoffman, West, \& Sheets, 2002). Similar to Chapman and colleagues, we found that the effect of the opt-out intervention was 
mediated by the appointment status of participants. Participants in the opt-out condition were more likely to have a vaccination appointment than participants in the opt-in condition, which increased the probability of getting vaccinated (Chapman et al., 2010). That is, the opt-out condition increased the likelihood of having an appointment for influenza vaccination, which in turn increased the probability of getting vaccinated.

Halpern, Ubel, and Asch (2007) have suggested that default options could be used to improve health care. Especially in the domain of organ donation, changing the default option has been shown to be effective. The number of registered organ donors is considerably larger in countries where people must opt-out from being registered if they do not wish to donate than in countries where people actively have to opt-in to be registered if they wish to donate (Johnson \& Goldstein, 2003). The Infectious Disease Society of America (IDSA) (2007) recommended a similar approach for annual influenza vaccination for HCWs. Halpern et al. (2007) suggested that the effect of the default procedure can be expected to be largest when people have a neutral attitude towards a health behavior and when it is not too easy to opt-out. Without strong preferences that guide a decision, people may be more likely to not act and to accept the default as the recommended behavior. Our previous studies suggest that most HCWs have a clear preference, both in favor or against influenza vaccination (Lehmann et al., 2015; Lehmann et al., 2014), which is likely to interfere with the default effect in this health domain. In addition, findings of previous studies had suggested that HCWs might build up more resistance against vaccination when their autonomy to choose is taken away (Lehmann, Ruiter, Wicker, van Dam, \& Kok, 2014). This is why we chose to make it fairly easy for them to opt-out by simply following a link in the invitation email and choosing the option to cancel the appointment. Even though this seemed necessary, it might additionally explain why the effects were not significant in our study.

Moreover, Li and Chapman (2013) proposed that the default procedure must be easily enforceable, which is the case for having an appointment, but when HCWs choose to not opt-out, they still have to remember their appointment, make time for it, and go to the vaccination location, which is not enforceable. Nevertheless, it is surprising that this relatively effortless and low-priced nudging strategy can show a difference in uptake that is comparable with the difference in uptake achieved by complex, multi-faceted campaigns to increase influenza vaccination uptake among HCWs (Looijmans-van den Akker et al., 2011; Riphagen-Dalhuisen et al., 2013).

A major strength of this study is the randomized experimental design that allowed for comparison of the two conditions while keeping the environment the same. However, the intervention location had the disadvantage of a modest sample size $(N=$ 122), which might have led to a too small power to detect an effect of condition on vaccination uptake. A post hoc power analysis with the program GPower (Erdfelder, Faul, \& Buchner, 1996) revealed a 0.28 power to detect a $12 \%$ absolute difference in vaccination uptake between the two groups, when $N=61$ per condition. Moreover, it has to be noted that because this study was executed in a tertiary care center of ex- 


\section{Chapter 7}

pertise for the diagnosis and treatment of patients with complex chronic organ failure, findings may not be generalizable to other health care settings.

\section{CONCLUSIONS}

In conclusion, even though we did not find an effect of the default option on influenza vaccination, being in the opt-out condition did increase the likelihood of HCWs to have an appointment for vaccination, which increased the likelihood of getting vaccinated. These findings suggest that using the default procedure could be a good alternative to the complex vaccination campaigns that have been proposed in recent years. This is especially the case because it is relatively easy to implement and it is low in cost, and as such even more cost-saving than other campaigns. However, since the achieved increases in vaccination rates are not nearly as high as proposed by Health Authorities, mandatory approaches should be considered. 
CHAPTER 8

General Discussion 



\section{GENERAL DISCUSSION}

The main objective of this dissertation was to gain more insight into the reasons why health care workers (HCWs) do (not) get vaccinated against influenza and to apply Intervention Mapping (IM) to the development of an intervention to promote influenza vaccination uptake among this group. Most of the studies described in this dissertation are part of the first step of the IM process. In this step, the Needs Assessment, the health problem, its consequences, and the social cognitive variables that influence the recommended behavior are analyzed (Bartholomew, Parcel, Kok, Gottlieb, \& Fernandez, 2011). For this purpose, past research on the topic is reviewed and on the basis of that, new evidence is generated under consideration of relevant theories. This final chapter discusses findings of the conducted studies, and describes the development and implementation of our behavior change program. Moreover, it summarizes the strengths and limitations of the studies and provides practical implications and future directions for research about influenza vaccination uptake among HCWs.

\section{Predictors of health care workers' decision to get vaccinated}

Past research had shown that HCWs have unfavorable attitudes towards influenza vaccination (Cohen \& Casken, 2012; Hofmann, Ferracin, Marsh, \& Dumas, 2006; Hollmeyer, Hayden, Poland, \& Buchholz, 2009). However, only a few studies included medical students and it is unknown when medical students develop these unfavorable attitudes (Christini, Schutt, \& Byers, 2007; Martinello, Jones, \& Topal, 2003; Talbot, Dellit, Hebden, Sama, \& Cuny, 2010). We therefore administered a questionnaire in a pre-clinical medical student population that suggested that knowledge gaps and negative attitudes towards influenza vaccination are already present before HCWs start their clinical careers (chapter 3 ).

To extend existing knowledge on facilitating and inhibiting factors of HCWs' vaccination uptake and to gain a more direct and in-depth insight into the social cognitive variables and underlying beliefs that guide their motivation to get vaccinated, we conducted one-on-one interviews with HCWs in Belgian, Dutch, and German hospitals (chapter 4). As was found in previous quantitative studies among HCWs, the most often reported reasons for vaccination were self-protection, patient protection, and protection of family members (Cohen \& Casken, 2012; Hofmann et al., 2006; Hollmeyer et al., 2009). The most often reported reasons against vaccination were fear of sideeffects or illness caused by the vaccine, a low risk perception to contract or transmit influenza, the disbelief in the effectiveness of the vaccine, organizational barriers, lack of knowledge, and undefined negative emotions resulting from the vaccination decision (Cohen \& Casken, 2012; Hofmann et al., 2006; Hollmeyer et al., 2009). We additionally identified three beliefs that are not commonly included in questionnaire studies of HCWs' decision to get vaccinated against influenza: the belief that other means 
of prevention are as effective or even more effective than influenza vaccination (prevention beliefs), the preference not to get vaccinated over getting vaccinated if one believes that the vaccine can cause illness (omission bias), and the belief that it is beneficial for the immune system to undergo illness (naturalistic beliefs) (Baron \& Ritov, 2004; Bridges, Kuehnert, \& Hall, 2003; Van den Dool et al., 2008). Moreover, the comparison of answers by HCWs from the three countries indicated some differences, which we further investigated in the study presented in chapter 5 . Findings of the online survey first of all suggested that HCWs can be categorized into three groups, on the basis of their motivation to get vaccinated against influenza: HCWs with no intention to vaccinate, the ones who did not form a clear intention, and HCWs with a strong positive intention to receive vaccination. We showed that these motivational states were in turn predicted by different social cognitive variables, additional beliefs, past experiences and demographics.

Furthermore, we found that attitude and past vaccination uptake explain a considerable amount of variance in the intention to get vaccinated against influenza, which is in line with previous research (Aguilar-Díaz, Jiménez-Corona, \& Ponce-deLeón-Rosales, 2011; Hollmeyer et al., 2009; Van den Dool et al., 2008). In addition to that, perceived negative social norms, having an omission bias, low moral norms, being older, having no patient contact, and being Belgian or Dutch compared to German increased the probability of no intention to get vaccinated compared to being unsure about vaccination. For HCWs with a high perceived susceptibility to contract influenza, low naturalistic views, and a lower motivation to solely get vaccinated for selfprotection, a strong positive intention was shown to be more likely than being unsure about vaccination.

Dutch HCWs scored overall highest on social cognitive variables and additional beliefs that contribute negatively to the intention to get vaccinated and showed the lowest vaccination uptake among the three countries. We therefore focused on Dutch HCWs in our longitudinal study (chapter 6), in which we investigated the same social cognitive variables, but included a follow-up measurement to test whether intention is a good predictor of the actual vaccination behavior of these HCWs. We again showed that HCWs can be categorized into three groups on the basis of their intention to get vaccinated against influenza, and attitude and past vaccination uptake again proved to be good predictors of intention. Findings from the Dutch sample suggested that HCWs with strong feelings of autonomy about the decision whether to get vaccinated were more likely to have no intention to get vaccinated against influenza, compared to HCWs who are unsure. Previous research showed that HCWs, independent of their immunization status, highly value the autonomy in regard to this decision (Hakim, Gaur, \& McCullers, 2011). This finding suggests that it might be beneficial to make HCWs aware of the consequences of their decision for patients. It has been suggested that these welfare concerns should outweigh HCWs autonomy in the decision whether to get vaccinated against influenza (Galanakis, Jansen, Lopalco, \& Giesecke, 2013). 
Additionally, HCWs who perceived vaccination as solely a matter of self-protection were more likely to have no intention than to be unsure about influenza vaccination. In this line, our findings further suggested the importance of promoting feelings of personal responsibility to protect patients by getting vaccinated to promote a positive attitude towards influenza vaccination, which predicts a more positive intention to get vaccinated.

The social cognitive variables identified in our studies, explained a considerable amount of variance in HCWs' intention to get vaccinated against influenza, which in turn explained a considerable amount of variance in the actual behavior of HCWs. Sheeran (2002) has shown that the average explained variance of intention-behavior relationships over a broad range of health behaviors to be $28 \%$. In our longitudinal study, intention accounted for $58 \%$ of the variance in behavior. Even though this is a good prediction, there is still $42 \%$ of unexplained variance that we do not understand and that HCWs might not be able to communicate. In the qualitative interview study, and both questionnaire studies (chapter 4, 5 and 6), consistently more unique predictors of no intention to get vaccinated against influenza, compared to being unsure, than for having a high intention were found. A possible explanation is that HCWs with a positive intention know exactly why they get vaccinated, while HCWs with no intention might be less clear about the reasons and as a consequence of this might agree more easily with the negative end of the utilized items in order to explain their unwillingness to get vaccinated. This is in accordance with the social psychological concept of cognitive dissonance. The cognitive dissonance theory states that individuals strive for internal consistency and that they are motivated to reduce dissonance by, for example, aligning their beliefs with their behavior (Festinger, 1962). In the case of influenza vaccination, dissonance might occur because of $\mathrm{HCWs}^{\prime}$ knowledge that influenza vaccination is recommended and that it is their occupational responsibility to protect patients. To be more consistent with their unwillingness to get vaccinated, HCWs may agree more easily with reasons for non-immunization.

Part of the unexplained variance might also be explained by the negative emotions that have been reported to result from the vaccination decision (chapter 4). This negative affect might be associated with a general mistrust and negative media attention that influenza vaccination has received, as well as the negative social norms that can be found in health care settings (Looijmans-van den Akker et al., 2009; Takayanagi, Cardoso, Costa, Araya, \& Machado, 2007), including the indifference of the management of some health care facilities to support the infection control measure of vaccinating HCWs against influenza. Understanding the source of the reported undefined negative feelings and uneasiness that result from the decision whether to get vaccinated might shed more light on this matter. HCWs in the other two categories - unsure and positive intention - should be more responsive to intervention programs intended to increase vaccination uptake. 


\section{The need for a new approach}

Our studies (chapter $3,5,6$ ) suggest that attitude is the most important predictor of HCWs' intention to get vaccinated against influenza. Together with past vaccination behavior, attitude explained a considerable amount of the variance in intention to get vaccinated. Looking at the predictors of attitude, we found that most of the social cognitive variables, as well as underlying beliefs included in our studies, contribute to the explanation of variance in attitude $\left(R^{2}=.89\right.$, chapter 5$)$. Therefore, the logical consequence would be to target these social cognitive variables and underlying beliefs to promote more positive attitudes. Step 3 of the IM process involves the identification of appropriate theoretical methods and practical applications that have been shown to change certain determinants and to reach program objectives (established in step 2). For attitude, these involve theoretical methods such as belief selection, persuasive communication, and repeated exposure (Bartholomew et al., 2011). All of these methods could be embedded into an educational program component.

Recent campaigns have almost all used educational approaches to increase the vaccination uptake of HCWs (Chittaro et al., 2009; Cooper \& O'Reilly, 2002; Doratotaj, Macknin, \& Worley, 2008; Llupià et al., 2010; Llupià et al., 2013; Riphagen-Dalhuisen et al., 2013; Salgado, Giannetta, Hayden, \& Farr, 2004). However, these programs did not show the intended effect. Interestingly, a recent study by Llupià and colleagues (2013) has shown that the intervention components they used had the intended effect on the determinants they focused on; knowledge and risk perception with regard to influenza increased among HCWs. However, this did not show an effect on the vaccination uptake of HCWs. The limited effects on behavior do not seem to be explained by a lack of intention to get vaccinated. Our longitudinal study (chapter 6 ) showed that $20 \%$ of the HCWs had a positive intention to get vaccinated, of which $74 \%$ did get vaccinated. Next to that, there is a group that does not have a clear preference whether to get vaccinated $(28 \%)$ and of those HCWs only $15 \%$ ultimately did get vaccinated. The remaining HCWs had no intention to get vaccinated and of the whole sample, fewer than $20 \%$ received influenza vaccination.

Consequently, there seems to be a need for an extra intervention component, or maybe even a radically different approach to change behavior, especially for HCWs who have a positive intention and for those that are still unsure. However, it should be considered that applying pressure will likely lead to defensive reactions (chapter 3 and 4). HCWs have been shown to value highly the autonomy in their decision whether to get vaccinated against influenza (Hakim et al., 2011). A method that has been shown to be effective in changing behavior without restricting the freedom of the decision maker is nudging (Goldstein, Johnson, Herrmann, \& Heitmann, 2008; Li \& Chapman, 2013). Nudges are small changes in the environment that push the decision maker to make a healthy decision, without actually restricting his/her decision autonomy. For our intervention, we brainstormed several different nudges, some of which are derived from 
social psychological principles that are known to influence behavior without much effort (i.e. social norms, incentives, implementation intentions), and some of which additionally make use of decision biases (i.e. framing effect, default effect) (Li \& Chapman, 2013).

Some of these nudges have previously been included in intervention programs to increase influenza vaccination uptake among HCWs. For instance, several interventions made use of social norms by publishing names or showing pictures of vaccinated staff (Llupià et al., 2010; Llupià et al., 2013; Riphagen-Dalhuisen et al., 2013), and some interventions have used incentives to stimulate vaccination uptake (Ajenjo, Woeltje, Babock, Gemeinhart, \& Jones, 2010; Doratotaj, Macknin, \& Worley, 2008; Llupià et al., 2010; Llupià et al., 2013). However, since they were embedded into programs with other components, it is unknown what their individual effect on vaccination uptake is.

Another nudge that we considered was the use of implementation intentions. Implementation intentions are action plans in which the individual specifies where, when, and how a behavior is executed to achieve a desired goal (Gollwitzer \& Sheeran, 2006). Milkman, Beshears, Choi, Laibson, and Madrian (2011) have tested the use of implementation intentions for influenza vaccination uptake in a large firm. Employees either received an email stating the times and locations for vaccination, or they received this email together with a prompt to write down the time and date when they were planning to get vaccinated. This resulted into an increase of the vaccination uptake. However, this strategy assumes an initial intention to get vaccinated, which is not always present among HCWs (chapter 5 and 6).

Research about framing effects suggests that decision makers perceive the same option very differently, depending on how it is framed (Li \& Chapman, 2013; Rothman \& Salovey, 1997). One promising application is to use the framing effect in combination with social norms. When framing social norm messages, research suggests that it is more effective to use descriptive social norm messages that are framed positively, instead of stating the negative social norm (Mollen, 2013). An example of a negative descriptive norm is: "The majority of HCWs do not get vaccinated against influenza." Alternatively, with health behaviors that are disregarded by the majority, it can be beneficial to communicate what people ought to do (injunctive norms), instead of what they do (Cialdini et al., 2006; Mollen, Ruiter, \& Kok, 2010).

Finally, a study by Chapman, Li, Colby, and Yoon (2010) used the default effect by sending e-mail appointments for annual influenza vaccination to University staff, which resulted in a $12 \%$ absolute difference in the vaccination rate, compared with staff that had to schedule their own appointment. Making annual influenza vaccination a default for HCWs has been recommended by the Infectious Disease Society of America (2007) and researchers in this field (Halpern, Ubel, \& Asch, 2007). After brainstorming several options for a new approach to increase influenza vaccination among HCWs, we considered the following components in collaboration with our planning group members: steering behavior with the opt-out default strategy, increasing knowledge and coun- 
teracting misconceptions, raising awareness of the social norm, and increasing the visibility of the vaccination status.

\section{Steering behavior with the opt-out default strategy}

Our main strategy to increase $\mathrm{HCWs}^{\prime}$ influenza vaccination uptake was to install the opt-out default procedure. From our studies, it becomes clear that there is a group of HCWs that has not yet formed a clear decision about influenza vaccination, or that has not translated their positive intentions into behavior. These HCWs might benefit from a scheduled appointment.

For this purpose, the default is manipulated by sending e-mail appointments for annual influenza vaccination to HCWs. HCWs in the opt-out condition get a prescheduled appointment by default and have to actively change or cancel it via a link in the e-mail if they do not want to have an appointment, while HCWs in the opt-in condition do not get an appointment and have to actively make an appointment if they want to have an appointment for vaccination. Personalized e-mails are sent with Qualtrics software, which automatically registers if participants retain, change, or cancel their appointment. This simplifies the sending of reminders, and ensures additional anonymity of the participating HCWs.

\section{Increasing knowledge and counteracting misconceptions}

Independent of the fact that the provision of information alone does not seem to add much to an increase in the vaccination uptake of HCWs, we wanted to provide the right information about the relevance of influenza vaccination, its safety, and the scientific evidence about influenza vaccination for the prevention of influenza and the transmission to patients. Llupià and colleagues (2013) showed that education does increase knowledge about influenza vaccination and that it heightens risk perceptions with regard to influenza. We therefore developed an educational presentation. In line with suggestions from Theories of Information Processing (Petty, Barden, \& Wheeler, 2009), the presented information contained good arguments in favor of influenza vaccination that are relevant for HCWs and not too distant from their beliefs. Moreover, information was included that was likely new to the audience (i.e. the prevalence of non-symptomatic influenza and transmission) and exposure to the main statements was repeated.

\section{Raising awareness of the social norm}

Two of our studies (chapter 3,4 ) had shown that the intention to get vaccinated against influenza was predicted by HCWs' perception that most colleagues do not get vaccinated against influenza. Social norms primarily play a role in situations in which people are unsure how to behave (Cialdini et al., 2006). The motivation to belong and to keep a positive self-image leads people to behave like the majority of people in their 
environment. Research on social norms shows that it is possible to regulate behavior by communicating the social norm belonging to the recommended behavior (Mollen, 2013). However, in situations in which the majority of people acts unhealthy (as in the example of influenza vaccination among HCWs), social norms can have a negative effect on others. In such a case, it is important not to communicate what the majority does (descriptive norm), but instead to communicate what people expect you to do (injunctive norm) (Cialdini et al., 2006; Mollen et al., 2010). In our case this means that we have to communicate social norms that imply that getting vaccinated against influenza is the recommended behavior. For this purpose, our planning group members decided to print posters with photos of medical staff leaders getting vaccinated against influenza, which were distributed on the different wards. Additionally, the posters depicted the social norm messages "Protect yourself and our patients with the flu shot.", and "I'm vaccinated; we are protected."

\section{Increasing the visibility of the vaccination status}

To make HCWs more aware of the social norm, we had additionally considered to make use of badges that are given to either immunized HCWs, or both immunizers and non-immunizers. In Switzerland, this approach has shown to lead to an increase in vaccination behavior. Iten, Bonfillon, Bouvard, Siegrist, and Pittet (2013) had given badges to vaccinated HCWs saying "I am vaccinated to protect you.", and HCWs who did not get vaccinated received a badge saying "I wear a mask to protect you." Wearing the badge was encouraged, but not mandatory. Despite the increase in the vaccination uptake of HCWs, this strategy was not used in the following years after introduction. A Dutch study did something similar by giving badges only to vaccinated HCWs, saying "Consciously vaccinated for you." ("Bewust geprikt voor u.") (RiphagenDalhuisen et al., 2013).

\section{Implementation of the strategy}

Two health care facilities agreed to participate as intervention settings: a tertiary care center of expertise for the diagnosis and treatment of patients with complex chronic organ failure, and a peripheral hospital, both in the south of the Netherlands. Additionally, another peripheral hospital agreed to act as control setting to the intervention hospital. Following an intensive planning phase in which several different planning group members from the intervention hospital were included (microbiology, infection control, communication department, human resources department), we agreed on implementing the opt-out default procedure, the educational presentation as part of regular educational seminars, and the posters with photos of vaccinated staff from the intervention hospital, which depicted social norm messages. We introduced the idea of using badges for vaccinated staff, but the idea was not well received and ultimately not utilized. 
Midway into the four-week period in which the HCWs where scheduled for influenza vaccination, the workers' union of our intervention hospital received a complaint by employees of the hospital who had been scheduled for influenza vaccination. We were asked to provide written comment on the union's concerns about privacy of employee data, not disclosing to HCWs that they were taking part in a study, and that the research project had been funded by a pharmaceutical company.

With regard to privacy of employees, we explained that it was agreed upon that only the executing researcher received and used email addresses of employees to ensure the highest possible anonymity of participants considering the kind of intervention used. We also stated that the online tool Qualtrics that had been used to send the personalized emails with appointments for vaccination was further protected by a password and guaranteed data confidentiality. Moreover, we were only interested in the number of people who would get vaccinated against influenza in the two conditions. We were not planning to further stratify the results by looking at any other information concerning the individual employees (i.e. age, gender, ward) that could have revealed their identity.

With regard to disclosing to HCWs that they were taking part in a study, we explained that we had communicated openly what the new procedure for annual influenza vaccination would be and also discussed this at a meeting for unit managers. However, fully disclosing to HCWs that they were randomly assigned to one of two conditions and that we hypothesized that this would lead to an increase in vaccination uptake for HCWs with an appointment for vaccination, would have likely influenced the effectiveness of the strategy. We explained that the study had been approved by a research ethics board and that not fully disclosing the studies' procedure conformed with standard research procedures in social psychological studies. Moreover, we explained that the choice whether to get vaccinated against influenza was still voluntary for HCWs.

With regard to concerns about funding by a pharmaceutical company, we explained that the project was funded by an unrestricted educational grant, which means that the funders neither had a role in the conception of the studies, the analyses or interpretation of the published data, nor did they have to approve the published studies. The acquired data are the property of Maastricht University, which has been established in a contract between the funders and the University. Despite convincing the board of directors of the fact that the study had been executed correctly, the workers' union urged us to stop the ongoing intervention, eventually leading to the director's decision to withdraw the permission to use the data generated by our study to avoid further conflict with the union.

Consequently, we can only report on our findings from the tertiary care center. Here we implemented the opt-out default procedure, randomly assigning half of the staff members to the opt-in condition and half of the staff members to the opt-out condition, and the educational presentation that could be attended by all staff mem- 
bers on a voluntary basis (chapter 7). We did not find a statistically significant increase in the likelihood of the opt-out condition on the probability to get vaccinated, because of limited power to detect an effect, shown by a post-hoc power analysis. Nevertheless, the difference of $11.5 \%$ in the influenza vaccination uptake rate between HCWs in the opt-out and in the opt-in condition is comparable with the difference reported by Chapman and colleagues (2010). We were additionally able to show that similar to findings by Chapman and colleagues, the effect of the opt-out intervention on vaccination uptake was mediated by the appointment status of participants. Participants in the opt-out condition were more likely to have a vaccination appointment than participants in the opt-in condition, which increased the probability of getting vaccinated.

\section{Strengths and limitations}

This dissertation has several strengths that should be highlighted. Existing knowledge about the factors that influence HCWs' decision whether to get vaccinated against influenza is largely based on post-hoc reasons for acceptance and refusal of the vaccine, while there is a limited number of studies that investigate theory-based predictors that might guide the motivation of HCWs. We therefore extended existing knowledge about social cognitive variables and beliefs that affect the motivation of HCWs to get vaccinated against influenza by combining findings from past research and theory to develop a questionnaire that is based on theoretical concepts and allows for multivariate analyses. Indeed, this led to the identification of factors that had not been associated with the intention to get vaccinated before: alternative prevention beliefs, the omission bias, and naturalistic beliefs. These beliefs contributed to the explanation of variance in the intention to get vaccinated, in addition to the determinants specified by the Reasoned Action Approach (Fishbein \& Ajzen, 2010). Moreover, two of our studies were conducted in three European countries, one of which had not been systematically surveyed before (Belgium). The studies showed that even though similar reasons for and against influenza vaccination have been reported for HCWs worldwide, there were differences in enabling and inhibiting social cognitive variables and additional beliefs that influenced the intention to get vaccinated of HCWs from neighboring countries.

Another strength of the studies we have conducted is the variety of methods we used. We combine knowledge from a qualitative online media analysis (chapter 2 ) that describes the communication sentiment regarding influenza vaccination of the general public, and a qualitative interview study among HCWs (chapter 4) that allows for a direct and in-depth analysis of the reasons and underlying beliefs for vaccination, with quantitative theory-based questionnaire studies that explore the predictive value of the social cognitive variables in explaining HCWs' intention to get vaccinated (chapter $5,6)$. Moreover our longitudinal study (chapter 6 ) included a follow-up survey, which made it possible to link intention with actual vaccination behavior. Intention proved to 
be a good predictor of HCWs' vaccination behavior, exceeding the average explained variance of intention-behavior relationships as stated in a meta-analysis by Sheeran (2002). Taken together, the studies described in this dissertation help to create a more complete picture of why the influenza vaccination coverage rates among European HCWs are so low, and they fill gaps that we identified in existing research about this topic.

Finally, another major strength of this dissertation is that we used a radically different approach to promote influenza vaccination uptake among HCWs, one that does not focus on the determinants of intention to get vaccinated or their reported reasons for vaccination. We thereby bypass the well-known intention-behavior gap that affects all health behaviors, by directly influencing vaccination behavior. Our intervention, even though underpowered and therefore not statistically significant, demonstrates an easy and cost-effective way that can potentially increase the influenza vaccination uptake of HCWs.

Next to the strengths, there are also several limitations to our studies that will be discussed here. The findings of studies in this dissertation that have been executed by means of qualitative methods (chapter 2 and 4), are descriptive in nature and do not enable us to make causal inferences about them, nor indicate relative importance of the different themes that emerged. Moreover, data collection and analysis was performed by only the first author, which could have biased the results and made it impossible to apply inter-rater reliability. Qualitative research is inherently interpretive and more coders could potentially decrease bias. However, coding was done in a systematic way by developing a coding scheme and all authors discussed the analysis process and interpretation of the data extensively so as to reduce bias to a minimum.

Moreover, due to the cross-sectional nature of the studies performed in chapters 3,4 , and 5 , no causal relationships could be established, nor the relative importance of social cognitive variables and beliefs in explaining why HCWs get or do not get vaccinated against influenza. In addition to that, the surveys included a limited number of questions, and some of the concepts were only measured with one item to reduce the length and to increase the response rate for an already time-pressured group of participants. Including more items could have improved the predictive power of our model.

In most of the studies, a response bias cannot be ruled out. The response rate of hospitals willing to participate was low and could have led to an overrepresentation of hospitals with an already well-established vaccination program, respectively a high vaccination coverage rate. The participating hospitals were chosen based on convenience, rather than representativeness of hospitals in the three different countries. Therefore, generalizations to the collective population of HCWs should be treated with caution. 


\section{Practical implications and future research}

The strategy that we developed and that is described in this dissertation, showed some potential to increase the influenza vaccination uptake among HCWs. We do think that it is suitable for implementation in hospitals, however some precautions should be made beforehand. One of the most important lessons learned is the importance of ensuring approval by and collaboration of the workers' union as planning group members from the start. Moreover, it should be communicated to the entire staff of the health care setting, several months before introduction of the opt-out default strategy, that there is a change in the usual procedure and that they will all receive a scheduled appointment for influenza vaccination to prevent the unrest we encountered. Additionally, it should be made clear that influenza vaccination will still be a voluntary choice for HCWs. From some of the replies on emails with pre-scheduled appointments, we learned that this aspect was not clear for everyone. When resistance can be reduced and HCWs get used to the new approach, this strategy will possibly show a higher impact. We recommend not only to introduce the opt-out default strategy into hospitals, but also to maintain this strategy, in order to be able to monitor how vaccination uptake changes over time.

Influenza vaccination should not be a topic that is only discussed once a year. Awareness of the risks of influenza, especially for people who are more likely to suffer from severe complications following an infection, and the importance of influenza vaccination in the prevention of nosocomial infections, and the transmission of influenza from HCWs to patients should be raised and regularly repeated through education. Our study among pre-clinical medical students (chapter 3 ), suggests that education about influenza and influenza vaccination in its current form might not be enough to create the right knowledge, and positive attitudes towards influenza vaccination. We therefore think that medical students and student nurses need to be adequately educated about influenza early in their medical training and this education should continue throughout the clinical careers of HCWs as a standard quality of care measure. Another possible target group that could be made more aware, next to the HCWs are the patients that enter health care settings. Educating patients about the importance of influenza vaccination and their risks to contract influenza through relatives, other patients, and HCWs might not only lead to a better self-protection, but also a heightened awareness towards the people they receive medical treatment from.

The ecological theory suggests that individual health behaviors can only be understood by considering the behaviors of the surrounding environments (Kok, Gottlieb, Commers, \& Smerecnik, 2008). This ecological approach is also one of the cornerstones of Intervention Mapping (Bartholomew et al., 2011; Kok, 2014). With regard to influenza vaccination, Kok and colleagues (2011) suggested the different ecological levels being the at risk patients, who are surrounded by HCWs as interpersonal environmental agents, which in turn are surrounded by the hospital or nursing home man- 
agement on the organizational level, which are surrounded by governmental policies. Most interventions focus on HCWs as target group, in combination with changes in the organization, such as reducing practical barriers and seeking support from the hospital management (Chittaro et al., 2009; Cooper \& O'Reilly, 2002; Lam, Chambers, Pierrynowski MacDougall, \& McCarthy, 2010; Llupià et al., 2010; Llupià et al., 2013; Riphagen-Dalhuisen et al., 2013; Salgado, Giannetta, Hayden, \& Farr, 2004). According to the ecological approach, interventions can be executed on any level (Kok et al., 2008). This means in practice, that interventions to increase influenza vaccination uptake among HCWs can for instance be targeted at patients, as stated above. One possibility would be to inform patients (belonging to the risk groups) about the importance of immunized HCWs in patient safety and to encourage patients to ask their attending HCW whether he/she is vaccinated against influenza and if necessary ask to be treated by a different physician or nurse. Probably less controversial would be to mobilize social support in the direct environment of HCWs. The use of so called vaccination champions - HCWs that actively promote influenza vaccination among their colleagues - has shown some positive effects (Talbot, Dellit, Hebden, Sama, \& Cuny, 2010). Intervention programs targeted on the organizational level, such as mobilizing management support and leadership-modelled programs also show some promising results on vaccination uptake (i.e., Hood \& Smith, 2009; Talbot et al., 2010). Lastly, it should be considered to change health care policies on the governmental level, which can be especially beneficial when it concerns nation-wide policies that are implemented in all hospitals (Maltezou et al., 2011; Palache, 2011). A policy that acknowledges the importance of influenza vaccination for infection control and patient safety may in the long run encounter less resistance from HCWs and health care organizations than behavior change programs imposed by external parties.

This also brings us to the point of discussion whether influenza vaccination should be voluntary or not. Given the low vaccination coverage rates and moderate effects of voluntary vaccination programs, it can be argued that a mandatory approach is necessary to achieve sufficient protection for patients. The debate whether influenza vaccination can be made a mandatory requirement for employment in health care facilities generates a lot of controversy in the scientific and medical field (Dubov \& Phung, in press; Galanakis et al., 2013; Lantos et al., 2010). Mandatory vaccination programs usually work with exemptions for HCWs who do not want to or cannot get vaccinated against influenza due to religious or medical reasons (Babcock, Gemeinhart, Jones, Dunagan, \& Woeltje, 2010; Quan et al., 2012; Rakita, Hagar, Crome, \& Lammert, 2010). HCWs who do not wish to get vaccinated, but fall outside such an exemption are usually required to wear a mask during influenza season, and in the strictest case are discharged from work (Babock et al., 2010; Quan et al., 2012). Opponents of making influenza vaccination mandatory for HCWs are mainly concerned about violations of civil liberties and that mandatory vaccination policies are violating the individual right to refuse treatment (Dubov \& Phung, in press; Finch, 2006; Galanakis et al., 2013). Advo- 
cates of a mandatory approach acknowledge the failure of voluntary programs to sufficiently ensure patient safety and argue that in the case of influenza vaccination of HCWs, welfare concerns outweigh autonomy concerns (Galanakis et al., 2013; Tilburt, Muellera, Ottenberga, Poland, \& Koeniga, 2008).

Hospitals in the U.S. that have mandatory vaccination programs report influenza vaccination coverage rates of $98 \%$ and higher, and a national survey study reported that the majority of infectious disease consultants support mandatory policies (Babock et al., 2010; Polgreen, Septimus, Talbot, Beekmann, \& Helms, 2010; Rakita et al., 2010). European HCWs are reported to largely oppose mandatory policies (Van Delden et al., 2008; Wicker, Marckmann, Poland, \& Rabenau, 2010). We propose for future research to compare the attitude towards influenza vaccination between HCWs working in a hospital with a vaccination mandate and those without a mandate. Research has shown that HCWs that have gotten vaccinated against influenza more frequently in the past, much more positively evaluate influenza vaccination and they are more likely to get vaccinated again (Looijmans-van den Akker et al., 2009; Takayanagi, Cardoso, Costa, Araya, \& Machado, 2007; Zhang, While, \& Norman, 2011). We therefore hypothesize that HCWs who are required to get vaccinated against influenza will adjust their opinion towards vaccination accordingly.

Two of our studies had been executed in neighboring European countries (chapter 3 and 4), and findings suggested that there are some differences with regard to social cognitive predictors of the intention of HCWs to get vaccinated against influenza. Even though there is no indication that nudges work differently in different countries, it might be beneficial to investigate whether the default effect is larger, respectively smaller in certain countries and why. Halpern and colleagues (2007) had suggested that using the default procedure for influenza vaccination would be easily implemented and unlikely be seen as controversial. However, we did encounter resistance that even led to the termination of the intervention in our hospital setting. Possibly, there is less resistance in the U.S., where it also gets more common for hospitals to employ vaccination mandates. When we look at existing intervention programs, we see that European countries in general seem to have lower influenza vaccination uptake rates than for instance the U.S., Canada, and Australia (Lam et al., 2010). Next to legal differences, there might be cultural differences that lead to the higher compliance rates outside of Europe. We recommend to explore these differences in different countries and to investigate whether the intention-behavior gap is smaller in countries with higher compliance rates. However, it is also possible that the efforts to increase the influenza vaccination uptake have been simply going on for a longer time, as suggested by Looijmans-van den Akker (2009).

Finally, our (social) media analysis (chapter 2) confirmed concerns about critical information regarding influenza vaccination on the Internet, which might influence the success of vaccination campaigns and Health Authority recommendations. Betsch, Renkewitz, Betsch, and Ulshöfer (2010) demonstrated that participants who visited 


\section{Chapter 8}

vaccine critical websites had an increased risk perception towards vaccination and a lower intention to get vaccinated. We therefore recommend the monitoring of online media coverage about influenza vaccination, especially on social media websites which showed considerably more criticism than news websites. As a consequence, effective communication strategies have to be developed to help e-health users, including HCWs to find reliable information about influenza and influenza vaccination on the Internet, and to counteract the enormous amount of negative media coverage that is currently available online. Betsch and colleagues (2012) recommend that public health websites should be easier to find, easy to use, and that they should provide the information, support, and advice with regard to vaccinations that e-health users are searching for.

\section{Concluding remarks}

This research project investigated the social cognitive variables that influence HCWs' decision whether to get vaccinated against influenza. The studies described in this dissertation suggest that HCWs can be categorized according to their intention to get vaccinated against influenza. Depending on their intention, different social cognitive variables are influential. However, the most influential determinant of a positive intention to get vaccinated seems to be a positive attitude towards influenza vaccination, which is in turn predicted by several other social cognitive variables. Instead of focusing on these determinants, we here proposed an alternative approach to promote influenza vaccination uptake among HCWs. With the opt-out default procedure, we showed an effortless and cost-effective nudging strategy to increase influenza vaccination uptake, which additionally has the advantage of offering an easy way to implement and maintain program use over a longer time-period for health care facilities without much external assistance. 
References 



\section{References}

Ablah, E., Konda, K., Tinius, T., Long, R., Vermie, G., \& Burbach, C. (2008). Influenza vaccine coverage and presenteeism in Sedgwick County, Kansas. American Journal of Infection Control, 36, 588-591.

Aguilar-Díaz, F. C., Jiménez-Corona, M. E., \& Ponce-de-León-Rosales, S. (2011). Influenza vaccine and healthcare workers. Archives of Medical Research, 42, 652-657.

Ahmed, F., Lindley, M. C., Allred, N., Weinbaum, C. M., \& Grohskopf, L. (2014). Effect of influenza vaccination of healthcare personnel on morbidity and mortality among patients: Systematic review and grading of evidence. Clinical Infectious Diseases, 58, 50-57.

Ajenjo, M. C., Woeltje, K. F., Babock, H. M., Gemeinhart, N., \& Jones, M. (2010). Influenza vaccination among healthcare workers: Ten-year experience of a large healthcare organization. Infection Control and Hospital Epidemiology, 31, 233-240.

Amodio, E., Restivo, V., Firenze, A., Mammina, C., Tramuto, F., \& Vitale, F. (2014). Can influenza vaccination coverage among healthcare workers influence the risk of nosocomial influenza-like illness in hospitalized patients? Journal of Hospital Infection, 86, 182-187.

Andreassen, H. K., Bujnowska-Fedak, M. M., Chronaki, C. E., Dumitru, R. C., Pudule, I., Santana, S., Voss, H., \& Wynn, R. (2007). European citizens' use of e-health services: A study of seven countries. BMC Public Health, 7, 53-59.

Asch, D. A., Baron, J., Hershey, J. C., Kunreuther, H., Meszaros, J., Ritov, I., \& Spranca, M. (1994). Omission bias and pertussis vaccination. Medical Decision Making, 14, 118-123.

Asur, S., \& Huberman, B. A. (2010). Predicting the future with social media. Retrieved from: http://www.arxiv.org arXiv:1003.5699v1.

Babcock, H. M., Gemeinhart, N., Jones, M., Dunagan, W. C., \& Woeltje, K. F. (2010). Mandatory influenza vaccination of health care workers: Translating policy to practice. Clinical Infectious Diseases, 50, 459464.

Baron, R. M., \& Kenny, D. A. (1986). The moderator-mediator variable distinction in social psychological research: Conceptual, strategic, and statistical considerations. Journal of Personality and Social Psychology, 51, 1173-1182.

Baron, J., \& Ritov, I. (2004). Omission bias, individual differences, and normality. Organizational Behavior and Human Decision Processes, 94, 74-85.

Bartholomew, L. K., Parcel, G. S., Kok, G., Gottlieb, N. H., \& Fernandez, M. E. (2011). Planning health promotion programs: An Intervention Mapping approach (3rd ed.). San Francisco: Jossey-Bass.

Bénet, T., Régis, C., Voirin, N., Robert, O., Lina, B., Cronenberger, S., Comte, S., Coppéré, B., \& Vanhems, P. (2012). Influenza vaccination of healthcare workers in acute-care hospitals: A case-control study of its effect on hospital-acquired influenza among patients. BMC Infectious Diseases, 12, 30-35.

Berland, G. K., Elliott, M. N., Morales, L. S., Algazy, J. I., Kravitz, R. L., Broder, M. S., Kanouse, D. E., Muñoz, J. A., Puyol, J.-A., Lara, M., Watkins, K. E., Yang, H., \& McGlynn, E. A. (2001). Health information on the internet. Accessibility, quality, and readability in English and Spanish. Journal of the American Medical Association, 285, 2612-2621.

Betsch, C., Brewer, N. T., Brocard, P., Davies, P., Gaissmaier, W., Haase, N., Leask, J., Renkewitz, F., Renner, B., Reyna, V. F., Rossmann, C., Sachse, K., Schachinger, A., Siegrist, M., \& Stryk, M. (2012). Opportunities and challenges of web 2.0 for vaccination decisions. Vaccine, 30, 3727-3733.

Betsch, C., Renkewitz, F., Betsch, T., \& Ulshöfer, C. (2010). The influence of vaccine-critical websites on perceiving vaccination risks. Journal of Health Psychology, 15, 446-455.

Betsch, C., \& Wicker, S. (2012). E-health use, vaccination knowledge and perception of own risk: Drivers of vaccination uptake in medical students. Vaccine, 30, 1143-1148.

Beyer, W. E. P., McElhaney, J., Smith, D. J., Monto, A. S., Nguyen-Van-Tam, J. S., \& Osterhaus, A. D. M. E. (2013). Cochrane re-arranged: Support for policies to vaccinate elderly people against influenza. Vaccine, 31, 6030-6033. 
Bijl, D., \& Schellekens, H. (2011). The sponsored pandemic of the Mexican flu? The International Journal of Risk and Safety in Medicine, 23, 73-79.

Blank, P. R., Schwenkglenks, M., \& Szucs, T. D. (2009). Vaccination coverage rates in eleven European countries during two consecutive influenza seasons. Journal of Infection, 58, 441-453.

Bridges, C. B., Kuehnert, M. J., \& Hall, C. B. (2003). Transmission of influenza: Implications for control in health care settings. Clinical Infectious Diseases, 37, 1094-1101.

Brodie, M., Flournoy, R. E., Altman, D. E., Blendon, R. J., Benson, J. M., \& Rosenbaum, M. D. (2000). Health information, the internet, and the digital divide. Health Affairs, 19, 255-265.

Brown, K. F., Long, S. J., Ramsay, M., Hudson, M. J., Green, J., Vincent, C. A., Kroll, J. S., Fraser, G., \& Sevdalis, N. (2012). UK parents' decision-making about measles-mumps-rubella (MMR) vaccine 10 years after the MMR-autism controversy: A qualitative analysis. Vaccine, 30, 1855-1864.

Burls, A., Jordan, R., Barton, P., Olowokure, B., Wake, B., Albon, E., \& Hawker, J. (2006). Vaccinating healthcare workers against influenza to protect the vulnerable - Is it a good use of healthcare resources? A systematic review of the evidence and an economic evaluation. Vaccine, 24, 4212-4221.

Centers for Disease Control and Prevention. (2010). Prevention and control of influenza with vaccines: Recommendations of the Advisory Committee on Immunization Practices (ACIP). Morbidity and Mortality Weekly Report, 59, 1-5.

Centers for Disease Control and Prevention. (2011). Immunization of health-care workers: Recommendations of the Advisory Committee on Immunization Practices (ACIP). Morbidity and Mortality Weekly Report, 60(RR07), 1-45.

Chapman, G. B., Li, M., Colby, H., \& Yoon, H. (2010). Opting in vs opting out of influenza vaccination. The Journal of the American Medical Association, 204, 43-44.

Chittaro, M., Turello, D., Calligaris, L., Farneti, F., Faruzzo, A., Fiappo, E., Panariti, M., \& Brusaferro, S. (2009). Impact of vaccinating HCWs on the ward and possible influence of avian flu threat. Infection, 37, 29-33.

Christini, A. B., Schutt, K. A., \& Byers, K. E. (2007). Influenza vaccination rates and motivators among healthcare worker groups. Infection Control and Hospital Epidemiology, 28, 171-177.

Cialdini, R. B., Demaine, L. J., Sagarin, B. J., Barrett, D. W., Rhoads, K., \& Winter, P. L. (2006). Managing social norms for persuasive impact. Social Influence, 1, 3-15.

Clark, J. P. (2003). How to peer review a qualitative manuscript. In Peer Review in Health Sciences (2nd ed.). London: BMJ Books.

Cline, R. J. W., \& Haynes, K. M. (2001). Consumer health information seeking on the internet: The state of the art. Health Education Research, 16, 671-692.

Cohen, D. L., \& Casken, J. (2012). Why are healthcare workers so resistant to the acceptance of influenza vaccine? A review of the literature to examine factors that influence vaccine acceptance. International Journal of Caring Sciences, 5, 26-35.

Cohen, J., Cohen, P., West, S., \& Aiken, L. (2003). Applied multiple regression/ correlation analysis for the behavioral sciences (3rd ed.). Mahwah, NJ: Lawrence Erlbaum.

Commission of the European Communities. (2009). Council recommendation on seasonal influenza vaccination. Brussels, 13.07.2009, retrieved from: http://ec.europa.eu/health/ph_threats/com/Influenza/docs/seasonflu_rec2009_en.pdf

Cooper, E., \& O'Reilly, M. (2002). A novel staff vaccination strategy. Infection Control and Hospital Epidemiology, 23, 232-233.

Costantino, C., Mazzucco, W., Azzolini, E., Baldini, C., Bergomi, M., Biafiore, A. D., \& Amodio, E. (2014). Influenza vaccination coverage among medical residents: An Italian multicentre survey. Human Vaccines and Immunotherapeutics, 10, 1204-1210.

Council of the European Union. (2009). Council recommendation of 22 December 2009 on seasonal influenza vaccination. Official Journal of the European Union (2009/1019/EU), retrieved from: http://www.epha.org/IMG/pdf/Council_Reccomendation_on_seasonal_flu_vaccine.pdf

Couto, C. R., Pannuti, C. S., Paz, J. P., Fink, M. C. D., Machado, A. A., de Marchi, M., \& Machado, C. M. (2012). Fighting misconceptions to improve compliance with influenza vaccination among health care workers: An educational project. PLOS ONE, 7, 1-6. 
Da Costa DiBonaventura, M., \& Chapman, G. B. (2008). Do decision biases predict bad decisions? Omission bias, naturalness bias, and influenza vaccination. Medical Decision Making, 28, 532-539.

Dolan, G. P., Harris, R. C., Clarkson, M., Sokal, R., Morgan, G., Mukaigawara, M., Horiuchi, H., Hale, R., Stormont, L., Béchard-Evans, L., Chao, Y.-S., Eremin, S., Martins, S., Tam, J. S., Peñalver, J., Zanuzdana, A., \& Nguyen-Van-Tam, J. S. (2012). Vaccination of health care workers to protect patients at increased risk for acute respitory disease. Emerging Infectious Diseases, 18, 1225-1234.

Dolan, G., Iredale, R., Williams, R., \& Ameen, J. (2004). Consumer use of the internet for health information: A survey of primary care patients. International Journal of Consumer Studies, 28, 147-153.

Doratotaj, S., Macknin, M. L., \& Worley, S. (2008). A novel approach to improve influenza vaccination rates among health care professionals: A prospective randomized controlled trial. American Journal of Infection Control, 36, 301-303.

Dubov, A., \& Phung, C. (in press). Nudges or mandates? The ethics of mandatory flu vaccination. Vaccine.

Dumitru, R. C., Bürkle, T., Potapov, S., Lausen, B., Wiese, B., \& Prokosch, H.-U. (2007). Use and perception of internet for health related purposes in Germany: Results of a national survey. International Journal of Public Health, 52, 275-285.

Elder, A. G., O’Donnell, B., McCruden, E. A. B., Symington, I. S., \& Carman, W. F. (1996). Incidence and recall of influenza in a cohort of Glasgow healthcare workers during the 1993-4 epidemic: Results of serum testing and questionnaire. British Medical Journal, 313, 1241-1242.

Elo, S., \& Kyngäs, H. (2008). The qualitative content analysis process. Journal of Advanced Nursing, 62, 107115.

Endrich, M. M., Blank, P. R., \& Szucs, T. D. (2009). Influenza vaccination uptake and socioeconomic determinants in 11 European countries. Vaccine, 27, 4018-4024.

Erdfelder, E., Faul, F., \& Buchner, A. (1996). GPower: A general power analysis program. Behavior Research Methods, Instruments, and Computers, 28, 1-11.

Eysenbach, G., \& Köhler, C. (2004). Health-related searches on the internet. Journal of the American Medical Association, 291, 2946.

Festinger, L. (1962). A theory of cognitive dissonance. Stanford, CA: Stanford University Press.

Finch, M. (2006). Point: Mandatory influenza vaccination for all health care workers? Seven reasons to say "No". Clinical Infectious Diseases, 42, 1141-1143.

Fishbein, M., \& Ajzen, I. (2010). Predicting and changing behavior: The reasoned action approach. New York, NY: Psychology Press.

Fox, S. (2011). The social life of health information. Retrieved from: http://www.pewinternet.org/files/oldmedia/Files/Reports/2011/PIP_Social_Life_of_Health_Info.pdf

Galanakis, E., Jansen, A., Lopalco, P. L., \& Giesecke, J. (2013). Ethics of mandatory vaccination for healthcare workers. Eurosurveillance, $18, \mathrm{pii}=20627$.

Geneesmiddelenbulletin. (2011). Werkzaamheid en effectiviteit van influenzavaccinatie. 45, 109-117.

Gesser-Edelsburg, A., Walter, N., \& Green, M. S. (2014). Health care workers - Part of the system or part of the public? Ambivalent risk perception in health care workers. American Journal of Infection Control, 42, 829-833.

Gezondheidsraad. (2011). Briefadvies Vaccinatie tegen seizoensgriep. 28 september 2011. Retrieved from: http://www.gezondheidsraad.nl/sites/default/files/201121Vaccinatie\%20tegen\%20seizoensgriep0.pdf

Gezondheidsraad. (2014). Grip op griep. Den Haag: Gezondheidsraad; publicatienr. 2014/16.

Gintis, H., Bowles, S., Boyd, R., \& Fehr, E. (2003). Explaining altruistic behavior in humans. Evolution and Human Behavior, 24, 153-172.

Glezen, W. P., Greenberg, S. B., Atmar, R. L., Piedra, P. A., \& Couch, R. B. (2000). Impact of respiratory virus infections on persons with chronic underlying conditions. The Journal of the American Medical Association, 283, 499-505.

Goldstein, D. G., Johnson, E. J., Herrmann, A., \& Heitmann, M. (2008). Nudge your customers toward better choices. Harvard Business Review, 86, 99-105.

Gollwitzer, P. M., \& Sheeran, P. (2006). Implementation intentions and goal achievement: A meta-analysis of effects and processes. Advances in Experimental Social Psychology, 38, 69-119. 
Gorse, G. J., O’Connor, T. Z., Young, S. L., Habib, M. P., Wittes, J., Neuzil, K. M., \& Nichol, K. L. (2006). Impact of a winter respiratory virus season on patients with COPD and association with influenza vaccination. Chest, 130, 1109-1116.

Grandinetti, D. A. (2000). Doctors and the web: Help your patients surf the net safely. Journal of Medical Economics, 77, 28-34.

Guthmann, J. P., Fonteneau, L., Ciotti, C., Bouvet, E., Pellissier, G., Lévy-Bruhl, D., \& Abiteboul, D. (2012). Vaccination coverage of health care personnel working in health care facilities in France: Results of a national survey, 2009. Vaccine, 30, 4648-4654.

Hakim, H., Gaur, A. H., \& McCullers, J. A. (2011). Motivating factors for high rates of influenza vaccination among healthcare workers. Vaccine, 29, 5963-5969.

Halpern, S. D., Ubel, P. A., \& Asch, D. A. (2007). Harnessing the power of default options to improve health care. The New England Journal of Medicine, 357, 1340-1344.

Hardey, M. (2001). 'E- health': The internet and the transformation of patients into consumers and producers of health knowledge. Information, Communication, and Society, 4, 388-405.

Harmsen, I. A. (2014). Vaccinating: Self-evident or not? Development of a monitoring system to evaluate acceptance of the National Immunization Program. Doctoral dissertation. Maastricht University: The Netherlands.

Hernández-García, I. (2012). Influenza vaccination rates and determinants among Spanish medical students. Vaccine, 31, 1-2.

Hofmann, F., Ferracin, C., Marsh, G., \& Dumas, R. (2006). Influenza vaccination of healthcare workers: A literature review of attitudes and beliefs. Infection, 34, 142-147.

Hollmeyer, H. G., Hayden, F., Poland, G., \& Buchholz, U. (2009). Influenza vaccination of health care workers in hospitals: A review of studies on attitudes and predictors. Vaccine, 27, 3935-3944.

Hood, J., \& Smith, A. (2009). Developing a "Best Practice" influenza vaccination program for health care workers - An evidence-based, leadership-modeled program. Professional Practice, 57, 308-312.

Hsu, J., Huang, J., Kinsman, J., Fireman, B., Miller, R., Selby, J., \& Ortiz, E. (2004). Use of e-health services between 1999 and 2002: A growing digital divide. Journal of the American Medical Informatics Association, 12, 164-171.

Impicciatore, P., Pandolfini, C., Casella, N., \& Bonati, M. (1997). Reliability of health information for the public on the World Wide Web: Systematic survey of advice on managing fever in children at home. British Medical Journal, 314, 1875-1879.

Iten, A., Bonfillon, C., Bouvard, T., Siegrist, C.-A., \& Pittet, D. (2013). P037: Nosocomial influenza prevention using multi-modal intervention strategies; 20-years of experience. Antimicrobial Resistance and Infection Control, 2, P37.

Jefferson, T., Di Pietrantonj, C., Al-Ansary, L. A., Ferroni, E., Thorning, S., \& Thomas, R. E. (2010). Vaccines for preventing influenza in the elderly. Cochrane Database of Systematic Reviews, 2. doi: 10.1002/14651858.CD004876.pub3.

Johnson, E. J., \& Goldstein, D. (2003). Do defaults save lives? Science, 302, 1338-1339.

Jones, R. (2002). Declining altruism in medicine. Understanding medical altruism is important in workforce planning. BMJ, 324, 624-625.

Kaplan, A. M., \& Haenlein, M. (2010). Users of the world, unite! The challenges and opportunities of social media. Business Horizons, 53, 59-68.

Kok, G. (2014). A practical guide to effective behavior change. How to apply theory- and evidence-based behavior change methods in an intervention. The European Health Psychologist, 16, 156-170.

Kok, G., Gottlieb, N. H., Commers, M., \& Smerecnik, C. (2008). The ecological approach in health promotion programs; A decade later. American Journal of Health Promotion, 22, 437-442.

Kok, G., van Essen, G. A., Wicker, S., Llupià, A., Mena, G., Correia, R., \& Ruiter, R. A. C. (2011). Planning for influenza vaccination in health care workers: An intervention mapping approach. Vaccine, 29, 85128519. 
Kummervold, P. E., Chronaki, C. E., Lausen, B., Prokosch, H. U., Rasmussen, J., Santana, S., Staniszewski, A., \& Wangberg, S. C. (2008). eHealth trends in Europe 2005-2007: A population-based survey. Journal of Medical Internet Research,10, e42.

Kuster, S. P., Shah, P. S., Coleman, B. L., Lam, P.-P., Tong, A., Wormsbecker, A., \& McGeer, A. (2011). Incidence of influenza in healthy adults and healthcare workers: A systematic review and meta-analysis. PLOS ONE, 6, e26239.

Lam, P.-P., Chambers, L. W., Pierrynowski MacDougall, D. M., \& McCarthy, A. E. (2010). Seasonal influenza vaccination campaign for the health care personnel: Systematic review. Canadian Medical Association Journal, 182, E542-E548.

Lantos, J. D., Jackson, M. A., Opel, D. J., Marcuse, E. K., Myers, A. L., \& Connelly, B. L. (2010). Controversies in vaccine mandates. Current Problems in Pediatric and Adolescent Health Care, 40, 38-58.

Lehmann, B. A., Ruiter, R. A. C., Chapman, G., \& Kok, G. (2014). The intention to get vaccinated against influenza and actual vaccination uptake of Dutch healthcare personnel. Vaccine, 32, 6986-6991.

Lehmann, B. A., Ruiter, R. A. C., van Dam, D., Wicker, S., \& Kok, G. (2015). Sociocognitive predictors of the intention of healthcare workers to receive the influenza vaccine in Belgian, Dutch and German hospital settings. Journal of Hospital Infection, 89, 202-209.

Lehmann, B. A., Ruiter, R. A. C., Wicker, S., van Dam, D., \& Kok, G. (2014). “I don't see an added value for myself": A qualitative study exploring the social cognitive variables associated with influenza vaccination of Belgian, Dutch and German healthcare personnel. BMC Public Health, 14, 407.

Li, M., \& Chapman, G. B. (2013). Nudge to health: Harnessing decision research to promote health behavior. Social and Personality Psychology Compass, 7, 187-198.

Llupià, A., García-Basteiro, A. L., Olivé, V., Costas, L., Ríos, J., Quesada, S., Varela, P., Bayas, J. M., \& Trilla, A. (2010). New interventions to increase influenza vaccination rates in health care workers. American Journal of Infection Control, 38, 476-481.

Llupià, A., Mena, G., Olivé, V., Quesada, S., Aldea, M., Sequera, V. G., Ríos, J., García-Basteiro, A. L., Varela, P., Bayas, J. M. \& Trilla, A. (2013). Evaluating influenza vaccination campaigns beyond coverage: A beforeafter study among health care workers. American Journal of Infection Control, 41, 674-678.

Looijmans-van den Akker, I. (2009). Increasing influenza vaccine uptake. A comprehensive approach. Doctoral dissertation. Utrecht University: The Netherlands.

Looijmans-van den Akker, I., Hulscher, M. E., Verheij, T. J. M., Riphagen-Dalhuisen, J., van Delden, J. J. M., \& Hak, E. (2011). How to develop a program to increase influenza vaccine uptake among workers in health care settings? Implementation Science, 6, 47-55.

Looijmans-van den Akker, I., van Delden, J. J. M., Verheij, T. J. M., van der Sande, M. A. B., van Essen, G. A., Riphagen-Dalhuisen, J., Hulscher, M. E., \& Hak, E. (2010). Effects of a multi-faceted program to increase influenza vaccine uptake among health care workers in nursing homes: A cluster randomised controlled trial. Vaccine, 28, 5086-5092.

Looijmans-van den Akker, I., van Delden, J. J. M., Verheij, Th. J. M., van Essen, G. A., van der Sande, M. A. B., Hulscher, M. E., \& Hak, E. (2009). Which determinants should be targeted to increase influenza vaccination uptake among health care workers in nursing homes? Vaccine, 27, 4724-4730.

Macesic, N., Kotsimbos, T. C., Kelly, P., \& Cheng, A. C. (2013). Hospital-acquired influenza in an Australian sentinel surveillance system. Medical Journal of Australia, 198, 370-372.

Machowicz, R., Wyszomirski, T., Ciechanska, J., Mahboobi, N., Wnekowicz, E., Obrowski, M., Zycinska, K., \& Zielonka, T. M. (2010). Knowledge, attitudes, and influenza vaccination of medical students in Warsaw, Strasbourg, and Teheran. European Journal of Medical Research, 2, 235-240.

MacKinnon, D. P., Lockwood, C. M., Hoffman, J. M., West, S. G., \& Sheets, V. (2002). A comparison of methods to test mediation and other intervening variable effects. Psychological Methods, 7, 83-104.

Maltezou, H. C., Maragos, A., Katerelos, P., Paisi, A., Karageorgou, K., Papadimitriou, T., \& Pierroutsakos, I. N. (2008). Influenza vaccination acceptance among health-care workers: A nationwide survey. Vaccine, 26, 1408-1410. 
Marquet, R. L., Bartelds, A. I., van Noort, S. P., Koppeschaar, C. E., Paget, J., Schellevis, F. G., \& van der Zee, J. (2006). Internet-based monitoring of influenza-like illness (ILI) in the general population of the Netherlands during the 2003-2004 influenza season. BMC Public Health, 6, 242-249.

Martinello, R. A., Jones, L., \& Topal, J. E. (2003). Correlation between healthcare workers' knowledge of influenza vaccine and vaccine receipt. Infection Control and Hospital Epidemiology, 24, 845-847.

McLennan, S., \& Wicker, S. (2010). Reflections on the influenza vaccination of healthcare workers. Vaccine, 28, 8061-8064.

Melén, K., Partinen, M., Tynell, J., Sillanpää, M., Himanen, S.-L., Saarenpää-Heikkilä, O., Hublin, C., Olsen, P., Ilonen, J., Nohynek, H., Syrjänen, R., Kilpi, T., Vuorela, A., Kirjavainen, T., Vaarala, O., \& Julkunen, I. (2013). No serological evidence of influenza A H1N1 pdm09 virus infection as a contributing factor in childhood narcolepsy after pandemrix vaccination campaign in Finland. PLOS ONE. doi: 10.1371/journal.pone.0068402.

Mertz, D., Kim, T. H., Johnstone, J., Lam, P.-P., Science, M., Kuster, S. P., Fadel, S. A., Tran, D., Fernandez, E., Bhatnagar, N., \& Loeb, M. (2013). Populations at risk for severe or complicated influenza illness: Systematic review and meta-analysis. British Medical Journal, 347, 1-16.

Milkman, K. L., Beshears, J., Choi, J. J., Laibson, D., \& Madrian, B. C. (2011). Using implementation intentions prompts to enhance influenza vaccination rates. Proceedings of the National Academy of Sciences, 108, 10415-10420.

Milunic, S. L., Quilty, J. F., Super, D. M., \& Noritz, G. H. (2010). Patterns of influenza vaccination among medical students. Infection Control and Hospital Epidemiology, 31, 85-88.

Molinari, N.-A. M., Ortega-Sanchez, I. R., Messonnier, M. L., Thompson, W. W., Wortley, P. M., Weintraub, E., \& Bridges, C. B. (2007). The annual impact of seasonal influenza in the US: Measuring disease burden and costs. Vaccine, 25, 5086-5096.

Mollen, S. (2013). Fitting in or breaking free? On health behavior, social norms and conformity. Doctoral dissertation. Maastricht University: The Netherlands.

Mollen, S., Ruiter, R. A. C., \& Kok, G. (2010). Current issues and new directions in psychology and health: What are the oughts? The adverse effects of using social norms in health communication. Psychology and Health, 25, 265-270.

McNab, C. (2009). What social media offers to health professionals and citizens. Bulletin of the World Health Organization, 87, 566-566. doi: 10.2471/BLT.09.066712

Nichol, K. L., Lind, A., Margolis, K. L., Murdoch, M., McFadden, R., Hauge, M., Magnan, S., \& Drake, M. (1995). The effectiveness of vaccination against influenza in healthy, working adults. The New England Journal of Medicine, 333, 889-893.

Nickerson, R. S. (1998). Confirmation bias: A ubiquitous phenomenon in many guises. Review of General Psychology, 2, 175-220.

Oh, H., Rizo, C., Enkin, M., \& Jadad, A. (2005). What is eHealth (3): A systematic review of published definitions. Journal of Medical Internet Research, 7, e1.

Palache, A. (2011). Seasonal influenza vaccine provision in 157 countries (2004-2009) and the potential influence of national public health policies. Vaccine, 29, 9459-9466.

Partinen, M., Saarenpää-Heikkilä, O., Ilveskoski, I., Hiblin, C., Linna, M., Olsén, P., Nokelainen, P., Alen, R., Wallden, T., Espo, M., Rusanen, H., Olme, J., Sätilä, H., Arikka, H., Kaipainen, P., Julkunen, I., \& Kirjavainen, T. (2012). Increased incidence and clinical picture of childhood narcolepsy following the 2009 H1N1 pandemic vaccination campaign in Finland. PLOS ONE, 7, e33723.

Patton, M. Q. (1990). Qualitative evaluation and research methods (2nd ed.). Newbury Park: Sage Publications.

Petty, R. E., Barden, J., \& Wheeler, S. C. (2009). The Elaboration Likelihood Model of Persuasion: Developing health promotions for sustainable behavioral change. In DiClemente, R. J., Crosby, R. A., \& Kegler, M. C. (Eds.). Emerging theories in health promotion practice and research (2nd ed., pp. 185-214). San Francisco: Jossey-Bass. 
Polgreen, P. M., Septimus, E., Talbot, T. R., Beekmann, S. E., \& Helms, C. (2010). Results of a national survey of infectious disease specialists regarding influenza vaccination programs for healthcare workers. Infection Control and Hospital Epidemiology, 31, 1063-1065.

Preacher, K. J., \& Hayes, A. F. (2004). SPSS and SAS procedures for estimating indirect effects in simple mediation models. Behavior Research Methods, Instruments, and Computers, 36, 717-31.

Preaud, E., Durand, L., Macabeo, B., Farkas, N., Sloesen, B., Palache, A., Shupo, F., \& Samson, S. I. (2014). Annual public health and economic benefits of seasonal influenza vaccination: A European estimate. BMC Public Health, 14, 813.

Qualman, E. (2011). Socialnomics. How social media transforms the way we live and do business. Ipswich, MA: Business Book Summaries.

Quan, K., Tehrani, D. M., Dickey, L., Spiritus, E., Hizon, D., Heck, K., Samuelson, P., Kornhauser, E., Zeitany, R., Mancia, S., Thrupp, L., Tiso, S. M., \& Huang, S. S. (2012). Voluntary to mandatory: Evolution of strategies and attitudes toward influenza vaccination of healthcare personnel. Infection Control and Hospital Epidemiology, 33, 63-70.

Rakita, R. M., Hagar, B. A., Crome, P., \& Lammert, J. K. (2010). Mandatory influenza vaccination of healthcare workers: A 5-year study. Infection Control and Hospital Epidemiology, 31, 881-888.

Riphagen-Dalhuisen, J., Burgerhof, J. G., Frijstein, G., van der Geest-Blankert, A. D., Danhof-Pont, M. B., de Jager, H. J., Bos, A. A., Smeets, E. E., de Vries, M. J., Gallee, P. M., \& Hak, E. (2013). Hospital-based cluster randomised controlled trial to assess effects of a multi-faceted programme on influenza vaccine coverage among hospital healthcare workers and nosocomial influenza in the Netherlands, 2009 to 2011. Eurosurveillance, 18, pii=20512.

Rondy, M., van Lier, A., van de Kassteele, J., Rust, L., \& de Melker, H. (2010). Determinants of HPV vaccine uptake in the Netherlands: A multilevel study. Vaccine, 28, 2070-2075.

Rothman, A. J., \& Salovey, P. (1997). Shaping perceptions to motivate healthy behavior: The role of message framing. Psychological Bulletin, 123, 3-19.

Salgado, C. D., Farr, B. M., Hall, K. K., \& Hayden, F. G. (2002). Influenza in the acute hospital setting. The Lancet Infectious Diseases, 2, 145-155.

Salgado C. D., Giannetta, E. T., Hayden, F. G., \& Farr, B. M. (2004). Preventing nosocomial influenza by improving the vaccine acceptance rate of clinicians. Infection Control and Hospital Epidemiology, 25, 923928.

Sartor, C., Tissot-Dupont, H., Zandotti, C., Martin, F., Roques, P., \& Drancourt, M. (2004). Use of a mobile cart influenza program for vaccination of hospital employees. Infection Control and Hospital Epidemiology, 25, 918-922.

Sartor, C., Zandotti, C., Romain, F., Jacomo, V., Simon, S., Atlan-Gepner, C., Sambuc, R., Vialettes, B., \& Drancourt, M. (2002). Disruption of services in an internal medicine unit due to a nosocomial influenza outbreak. Infection Control and Hospital Epidemiology, 23, 615-619.

Schmitt-Beck, R. (2003). Mass communication, personal communication and vote choice: The filter hypothesis of media influence in comparative perspective. British Journal of Political Science, 33, 233-259.

Schnirring, L. (2007, January 25). IDSA urges requiring flu shots for healthcare workers. Center for Infectious Disease Research and Policy. Retrieved from: http://www.cidrap.umn.edu/news-pespetive/2007/01 /idsa-urges-requiring -flu-shots-healthcare-workers

Sheeran, P. (2002). Intention-behavior relations: A conceptual and empirical review. In Strobe, W., Hewstone, M., editors. European review of social psychology, vol. 12 (pp. 1-30). Chichester, UK: Wiley.

Smedley, J., Poole, J., Waclawski, E., Stevens, A., Harrison, J., Watson, J., Hayward, A., \& Coggon, D. (2007). Influenza immunization: Attitudes and beliefs of UK healthcare workers. Occupational and Environmental Medicine, 64, 223-227.

Steinhoff, M. C., Omer, S. B., Roy, E., El Arifeen, S., Raqib, R., Dodd, C., Breiman, R. F., \& Zaman, K. (2012). Neonatal outcomes after influenza immunization during pregnancy: A randomized controlled trial. $\mathrm{Ca}$ nadian Medical Association Journal, 184, 645-653. 
Takayanagi, I. J., Cardoso, M. R. A., Costa, S. F., Araya, M. E. S., \& Machado, C. M. (2007). Attitudes of health care workers to influenza vaccination: Why are they not vaccinated? American Journal of Infection Control. doi:10.1016/j.ajic.2006.06.002

Talbot, T. R. (2014). Update on immunizations for healthcare personnel in the United States. Vaccine, 32, 4869-4875.

Talbot, T. R., Dellit, T. H., Hebden, J., Sama, D., \& Cuny, J. (2010). Factors associated with increased healthcare worker influenza vaccination rates: Results from a national survey of university hospitals and medical centers. Infection Control and Hospital Epidemiology, 31, 456-462.

The Lancet. (2010). Retraction-lleal-lymphoid-nodular hyperplasia, non-specific colitis, and pervasive developmental disorder in children. The Lancet, 375, 445.

Thomas, D. R. (2006). A general inductive approach for analyzing qualitative evaluation data. American Journal of Evaluation, 27, 237-246.

Thomas, R. E., Jefferson, T., \& Lasserson, T. J. (2010). Influenza vaccination for healthcare workers who work with the elderly. Cochrane Database Systematic Review, 2, CD005187.

Tilburt, J. C., Muellera, P. S., Ottenberga, A. L., Poland, G. A., \& Koeniga, B. A. (2008). Facing the challenges of influenza in healthcare settings: The ethical rationale for mandatory seasonal influenza vaccination and its implications for future pandemics. Vaccine, 26S, D27-D30.

Van Delden, J. J. M., Ashcroft, R., Dawson, A., Marckmann, G., Upshur, R., \& Verweij, M. F. (2008). The ethics of mandatory vaccination against influenza for health care workers. Vaccine, 26, 5562-5566.

Van den Dool, C., Bonten, M. J., Hak, E., \& Wallinga, J. (2009). Modeling the effects of influenza vaccination of health care workers in hospital departments. Vaccine, 27, 6261-6267.

Van den Dool, C., van Strien, A. M., Looijmans-van den Akker, I., Bonten, M. J. M., Sanders, E. A., \& Hak, E. (2008). Attitude of Dutch hospital personnel towards influenza vaccination. Vaccine, 26, 1297-1302.

Van der Sande, M. A. B., Jacobi, A., Meijer, A., Wallinga, J., van der Hoek, W., \& van der Lubben, M. (2012). The 2009 influenza A (H1N1) pandemic. Management and vaccination strategies in the Netherlands. Bundesgesundheitsblatt, 56, 67-75.

Van Essen, G. A., Palache, A. M., Forleo, E., \& Fedson, D. S. (2003). Influenza vaccination in 2000: Recommendations and vaccine use in 50 developed and rapidly developing countries. Vaccine, 21, 1780-1785.

Vasterman, P. L. M., \& Ruigrok, N. (2013). Pandemic alarm in the Dutch media: Media coverage of the 2009 influenza $A(\mathrm{H} 1 \mathrm{~N} 1)$ pandemic and the role of the expert sources. European Journal of Communication, 28, 436-453.

Voirin, N., Barret, B., Metzger, M. H., \& Vanhems, P. (2009). Hospital-acquired influenza: A synthesis using the Outbreak Reports and Intervention Studies of Nosocomial Infection (ORION) statement. Journal of Hospital Infection, 71, 1-14.

Wakefield, A. J., Murch, S. H., Anthony, A., Linnell, J., Casson, D. M., Malik, M., Berelowitz, M., Dhillon, A. P., Thomson, M. A., Harvey, P., Valentine, A., Davies S. E., \& Walker-Smith, J. A. (1998). Ileallymphoidnodular hyperplasia nonspecific colitis and pervasive developmental disorder in children. The Lancet, 351, 637-641.

Weingarten, S., Riedinger, M., Burnes Bolton, L., Miles, P., \& Ault, M. (1989). Barriers to influenza vaccine acceptance. A survey of physicians and nurses. American Journal of Infection Control, 17, 202-207.

WHO Committee Report (2013). Global Advisory Committee on Vaccine Safety, 12-13 June 2013, Geneva, World Health Organization (Weekly Epidemiological Record No. 29).

WHO Fact Sheets (2009). Influenza (Seasonal), Geneva, World Health Organization (Fact Sheet No. 211).

WHO Fact Sheets (2014). Influenza (Seasonal), Geneva, World Health Organization (Fact Sheet No. 211).

WHO Position Paper (2005). Influenza vaccines, Geneva, World Health Organization (Weekly Epidemiological Record No. 33).

WHO Position Paper (2012). Vaccines against influenza WHO position paper - November 2012, Geneva, World Health Organization (Weekly Epidemiological Record No. 33).

Wicker, S., Marckmann, G., Poland, G. A., \& Rabenau, H. F. (2010). Healthcare workers' perceptions of mandatory vaccination: Results of an anonymous survey in a German university hospital. Infection Control and Hospital Epidemiology, 31, 1066-1069. 
Wicker, S., Rabenau, H. F., Doerr, H. W., \& Allwinn, R. (2009). Influenza vaccination compliance among health care workers in a German university hospital. Infection, 37, 197-202.

Wicker, S., Rabenau, H. F., von Gierke, L., François, G., Hambach, R., \& De Schryver, A. (2013). Hepatitis B and influenza vaccines: Important occupational vaccines differently perceived among medical students. Vaccine, 13, 5111-5117.

Wongsurakiat, P., Maranetra, K. N., Wasi, C., Kositanont, U., Dejsomritrutai, W., \& Charoenratanakul, S. (2004). Acute respiratory illness in patients with COPD and the effectiveness of influenza vaccination: $A$ randomized controlled study. Chest, 125, 2011-2020.

World Health Organization. (2002). Guidelines on prevention and control of hospital associated infections. New Delhi: World Health Organization.

Wright, D. K., \& Hinson, M. D. (2009). An updated look at the impact of social media on public relations practice. Public Relations Journal, 3, 1-27.

Zhang, J., While, A. E., \& Norman, I. J. (2012). Seasonal influenza vaccination knowledge, risk perception, health beliefs and vaccination behaviours of nurses. Epidemiology and Infection, 9, 1569-1577.

Zimmerman, R. K., Santibanez, T. A., Janosky, J. E., Fine, M. J., Raymund, M., Wilson, S. A., Bardella, I. J., Medsger, A. R., \& Nowalk, M. P. (2003). What affects influenza vaccination rates among older patients? An analysis from inner-city, suburban, rural, and veterans affairs practices. American Journal of Medicine, 114, 31-38.

Zimmerman, R., Wolfe, R., Fox, D., Fox, J., Nowalk, M., Troy, J., \& Sharp, L. (2005). Vaccine criticism on the world wide web. Journal of Medical Internet Research, 7. 

Valorization 



\section{Valorization addendum}

The research project presented in this dissertation has a clear social and economic relevance, in addition to the scientific relevance. As is explained in the dissertation, national and international Health Authorities, as well as researchers from different fields have identified the problem of low influenza vaccination uptake rates among health care workers (HCWs) and recommend annual vaccination of this group. In short, the reasons why influenza vaccination uptake has to be higher among HCWs are:

1) Influenza vaccination uptake of HCWs prevents the transmission of influenza to vulnerable patients and thereby decreases morbidity and mortality associated with influenza.

2) Influenza vaccination uptake protects HCWs, who have a more than three times higher risk of contracting influenza.

3) Influenza vaccination uptake of HCWs will reduce the economic burden of annual influenza epidemics by reducing costs associated with influenza-related medical treatments and work-absenteeism.

Furthermore, influenza vaccination is effective, cheap, safe, and easy to apply. The people who suffer the most from the low vaccination uptake of HCWs are the vulnerable patients they care for. Over and above having an increased risk to suffer from severe, sometimes life-threatening consequences following infection with influenza, people with a weakened immune system have been shown to be less well protected by influenza vaccination themselves. They therefore need the extra protection from people in their environment - so-called herd-immunity. They should not have to worry whether it is safe for them to receive medical treatment.

Despite existing programs to increase uptake, influenza vaccination coverage rates stay too low. We therefore investigated the social-cognitive variables associated with HCWs' motivation to get vaccinated in three European countries and developed a costeffective behavior change program to promote influenza vaccination uptake among this group. Moreover, the program can easily be implemented and maintained by hospitals without much external assistance. A vaccination program that is easy to implement and successful in increasing vaccination rates is in the interest of:

- Patients and their relatives, who have a decreased risk of acquiring influenza in the hospital;

- HCWs, their colleagues, and their relatives, because they also have a decreased risk to get infected with influenza;

- Occupational health physicians (or other people responsible for offering annual influenza vaccination) who are looking for a way to promote influenza vaccination uptake among their HCWs;

- Hospitals (and other health care facilities), because they can increase patient safety, reduce costs associated with influenza outbreaks, and decrease influenza-related work-absenteeism; 


\section{Valorization}

- And Health Authorities and governments, because vaccination pursues their goal of decreasing morbidity, mortality, and socioeconomic costs associated with annual influenza epidemics.

The developed program can be called innovative, because it is a radically different approach compared to the existing programs. Most of the existing programs are complex, multifaceted interventions that require much effort to implement and are basically impossible to maintain by the health care facilities without external assistance. Our program included only two components - education through a presentation and personalized email appointments for influenza vaccination sent with an online tool. They are easy to implement and the only extra costs generated are those of ordering enough vaccines so that everybody who wishes to get vaccinated can get vaccinated.

This dissertation will amongst others be made available to the academic community, health care providers, such as hospitals, public health agencies, and Abbott Health Care Products B. V., who funded this research project with an unrestricted educational grant. Thereby, the insights we established in this project will be distributed and shared, which will in the ideal case lead to continuation of research in this field as proposed in chapter 8 of this dissertation, and to the implementation and maintenance of the proposed behavior change program. 
Summary 



\section{Summary}

Annual influenza epidemics are a major public health problem causing considerable morbidity and mortality. Especially affected are people belonging to the risk groups young children, the elderly, and people with underlying chronic diseases. Health care workers are recommended to get vaccinated against influenza annually, because it has been shown to reduce the transmission of influenza to vulnerable patients and to decrease health care costs. Despite the evidence for the effectiveness of vaccination in the prevention of hospital-acquired influenza, decreasing work-absenteeism, and reducing costs, vaccination coverage rates are low among HCWs. The aim of this research project was to extend current knowledge about why HCWs are (un-) willing to get vaccinated and to develop a theory- and evidence-based behavior change program to promote influenza vaccination uptake among this group. The presented studies cover the communication sentiment regarding influenza vaccination on the Internet, predictors of pre-clinical medical students' and HCWs' intention to get vaccinated, and actual vaccination uptake, as well as the behavior change program that was implemented in a tertiary care center.

Chapter $\mathbf{2}$ describes a study investigating the Internet coverage of influenza and influenza vaccination on Dutch news sites and social media websites. The aim of this study was to gain insight into the communication with regard to influenza during a three months period in which an influenza epidemic took place and to examine how communication differs between news sites and social media websites. Three overarching themes have been consistently found in the information presented in both media sources: (1) the influenza epidemic, (2) general information about the virus, prevention and treatment, and (3) uncertainty and mistrust regarding influenza vaccination. Coverage of these topics is roughly the same between the media sources, but communication differs in message tone between the two sources. News sites tended to be more neutral and non-judgmental in their reports, while social media websites showed a more critical evaluation of the harmfulness of influenza and the necessity of influenza vaccination. Media may influence people's decision making with regard to influenza vaccination and may thereby influence the success of vaccination campaigns and recommendations by Health Authorities.

Chapter $\mathbf{3}$ presents a questionnaire study among pre-clinical medical students at a German University hospital. The purpose of this study was to gain insight into factors that predict medical students' intention to get vaccinated against influenza, as well as the reasons for acceptance and refusal of the vaccine. Findings showed that, compared to medical students with uncertain intentions, those with no intention to get vaccinated against influenza were less likely to think that important others (i.e. family, colleagues) would expect them to get vaccinated, and they were more likely to have strong feelings of autonomy with regard to the decision whether to get vaccinated. Compared to those with an uncertain intention, medical students with a positive inten- 
tion to get vaccinated showed a positive attitude towards influenza vaccination and also strong feelings of autonomy with regard to the decision whether to get vaccinated. Reasons for acceptance and refusal of influenza vaccination were similar to findings from studies with HCWs. The most commonly stated reasons to get vaccinated were self-protection, patient protection, and protection of family and friends. The most commonly stated reasons for refusal of the vaccine were a low risk perception (low susceptibility and severity) to contract influenza, fear of side-effects, disbelief in the effectiveness of the vaccine, and that vaccination was never offered. These results suggest that education about influenza and the importance of vaccination should be addressed early in medical training and hospitals should make vaccines accessible for their students.

Chapter 4 describes a qualitative study in which 123 Belgian, Dutch and German HCWs were interviewed about their reasons in favor and against influenza vaccination, their intention to get vaccinated, and possible barriers to vaccination. The aim was to explore the determinants and beliefs associated with the decision to get vaccinated against influenza and to gain a direct and more in-depth understanding of these determinants than could be captured by quantitative studies. Moreover, the similarities and differences of HCWs from three neighboring countries were explored. Across countries, self-protection, patient protection, and protection of family members were reported as the most important reasons to get vaccinated. Reasons not to get vaccinated were fear of side-effects, low risk perception, belief in the ineffectiveness of influenza vaccination, organizational barriers, a number of misconceptions, and undefined negative emotions. We identified three beliefs that had not been extensively described before with regard to this topic: alternative prevention beliefs, the omission bias, and naturalistic views. The determinants and beliefs found in this study have to be investigated quantitatively in order to identify their relative and combined importance in explaining the intention to get vaccinated of HCWs, as well as the importance of the similarities and differences between the three countries.

Chapter 5 describes an online questionnaire study that investigated the relative and combined strength of predictors from past research, theory and our qualitative study (chapter 4), in explaining the motivation to get vaccinated against influenza of Belgian, Dutch, and German HCWs. Moreover, this study provides insight into whether there is a need for country-specific interventions to increase influenza vaccination coverage among HCWs. Results suggested that there are three groups of HCWs that are influenced by different determinants: HCWs with no intention to get vaccinated, HCWs who are unsure about vaccination, and HCWs who have a strong positive intention to get vaccinated. Attitude and past vaccination uptake explained a considerable amount of variance in the intention to get vaccinated against influenza. Moreover, perceived negative social norms, having an omission bias, low moral norms, being older, and having no patient contact increased the probability of no intention to get vaccinated compared to being unsure about vaccination. HCWs were more likely to 
have a positive intention rather than being unsure about vaccination if they perceived high susceptibility to contract influenza, held low naturalistic views, and stated a lower motivation to get vaccinated solely for self-protection. Findings further suggest that Belgian HCWs score overall highest on facilitators of vaccination intention, while Dutch HCWs score highest on the inhibiting determinants. Therefore, country-specific interventions and a focus on different determinants depending on HCWs intention to get vaccinated could be effective in promoting vaccination uptake.

Chapter 6 describes an online questionnaire study that utilizes the determinants of the intention to get vaccinated against influenza (described in chapter 5 ) in a Dutch sample of HCWs. Then it is tested whether the intention to get vaccinated, as well as the determinants, are good predictors of the actual vaccination uptake of these HCWs with a follow-up survey. The aim was to identify the determinants that are most likely to help increase the influenza vaccination uptake among HCWs. HCWs with a strong positive intention to get vaccinated against influenza were more likely to have a positive attitude towards influenza vaccination and they tended to have had more influenza vaccinations in the past. HCWs with no intention to get vaccinated showed a higher probability of having a negative attitude, strong feelings of autonomy in the decision whether to get vaccinated, showed an omission bias, lower feelings of personal responsibility to protect patients through vaccination, higher self-protection motives, and they tended to not have been vaccinated in the past. The included determinants contributed largely to the explanation of HCWs' intention to get vaccinated against influenza, and intention was in turn a strong predictor of actual vaccination behavior.

Chapter 7 assessed a strategy to increase the influenza vaccination coverage rate among Dutch employees of a tertiary care center of expertise for the diagnosis and treatment of patients with complex chronic organ failure. The primary aim of the study was to find out if the vaccination rate among HCWs can be improved by manipulating the default option: half of the HCWs received an e-mail with an appointment for vaccination, with the possibility to cancel or change the appointment via a link in the email (opt-out condition), while the other half received an email explaining that there were free vaccines available and that they could schedule an appointment for vaccination (opt-in condition). The findings show no statistically detectable effect of condition on being vaccinated against influenza, probably due to a lack of statistical power to detect an effect. However, the difference of $11.5 \%$ in vaccination uptake between the conditions is comparable with another study that had used this approach to promote influenza vaccination. Moreover, HCWs in the opt-out condition were more likely to have an appointment for influenza vaccination, which in turn increased the probability of getting vaccinated. It is suggested that using the opt-out default procedure could be a good alternative to the complex vaccination campaigns that have been proposed in recent years. The developed strategy is relatively easy to implement, low in cost, and might still show similar results as complex campaigns when executed under right circumstances. 


\section{Summary}

Chapter 8 summarizes the findings of the studies and describes the development and implementation of the proposed behavior change program to promote influenza vaccination uptake among HCWs. It gives an overview of the strengths and limitations of the research project and discusses practical implications and recommendations for future research. In particular, it is recommended to implement the opt-out default strategy in hospital settings and to monitor its impact over a longer time period. For it to be successful, procedural changes have to be communicated to HCWs and raising awareness about the importance of influenza vaccination should be a recurrent educational effort. Moreover, it is proposed that behavior change programs could be developed that focus on target groups surrounding and influencing HCWs indirectly, such as patients, hospital managements, and governments. However, it is also suggested that mandatory vaccinations programs be considered, as voluntary programs have not shown the intended effect on vaccination uptake. Finally, it is recommended to monitor (social) media reports about influenza vaccination, to facilitate the use of public health websites, and to develop communication strategies to counteract the negative media attention that influenza vaccination receives. 
Samenvatting 



\section{Samenvatting}

Jaarlijkse influenza epidemieën zijn een ernstig probleem voor de volksgezondheid en veroorzaken aanzienlijke morbiditeit en mortaliteit. Ze zijn vooral problematisch voor mensen behorend tot de risicogroepen - jonge kinderen, ouderen en mensen met onderliggende chronische ziektes. Gezondheidswerkers worden geadviseerd zich elk jaar tegen influenza te laten vaccineren omdat dit tot een reductie in de overdracht van influenza naar kwetsbare patiënten leidt en kosten in de gezondheidszorg vermindert. Ondanks de evidentie voor de effectiviteit van vaccinatie in het voorkomen van infecties opgelopen binnen het ziekenhuis, het verminderen van werkverzuim, en het verminderen van kosten, blijven vaccinatiegraden onder gezondheidswerkers laag. Het doel van dit onderzoeksproject was om de huidige kennis over de redenen waarom gezondheidswerkers (niet) gevaccineerd willen worden uit te breiden en om een op theorie- en bewijs gebaseerd gedragsveranderingsprogramma te ontwikkelen waardoor influenzavaccinatie verhoogd wordt. De gepresenteerde studies gaan over de communicatie over influenzavaccinatie op het internet, voorspellers van de intentie om zich te laten vaccineren, evenals daadwerkelijk vaccinatiegedrag van preklinische geneeskunde-studenten en gezondheidswerkers, en de implementatie van het gedragsveranderingsprogramma in een tertiaire zorginstelling.

Hoofdstuk 2 beschrijft een studie die de op Nederlandse nieuwssites en sociale media websites geplaatste berichten met betrekking tot influenza en influenzavaccinatie onderzoekt. Het doel van de studie was om inzicht te krijgen in de communicatie over influenza tijdens de drie maanden waarin een epidemie plaatsvond en om uit te vinden in hoeverre communicatie op nieuwssites en op sociale media websites van elkaar verschilt. Drie overkoepelende onderwerpen kwamen in de communicatie op beide media soorten herhaaldelijk naar voren: (1) de influenza epidemie, (2) algemene informatie over het virus, preventie en behandeling, en (3) onzekerheid en wantrouwen ten aanzien van influenzavaccinatie. De omvang van deze onderwerpen was vergelijkbaar tussen de mediabronnen maar het sentiment van de communicatie verschilde in de twee bronnen. Nieuwssites zijn neutraler en minder beoordelend in hun rapporten, terwijl sociale media websites meer kritische evaluatie van de ernst van influenza en de noodzakelijkheid van influenzavaccinatie tonen. Media zouden de beslissing met betrekking tot influenzavaccinatie kunnen beïnvloeden en daardoor ook het succes van vaccinatiecampagnes en adviezen door gezondheidsautoriteiten.

Hoofdstuk 3 presenteert een vragenlijststudie onder preklinische geneeskundestudenten van een academisch ziekenhuis in Duitsland. Het doel van deze studie was om meer inzicht te krijgen in de factoren die de intentie van geneeskundestudenten om zich tegen influenza te laten vaccineren voorspellen, evenals de beweegredenen voor acceptatie en weigering van het vaccin. Het onderzoek laat zien dat vergeleken met geneeskundestudenten met een ongewisse intentie, het voor studenten zonder intentie om zich tegen influenza te laten vaccineren minder waarschijnlijk was om te 
denken dat belangrijke anderen (bijv. familie, collega's) van hen verwachten dat ze zich laten vaccineren. Studenten zonder intentie hadden ook een sterker gevoel van autonomie in de beslissing om zich te laten vaccineren. Vergeleken met diegenen die een ongewisse intentie hebben, hadden geneeskundestudenten met een positieve intentie om zich te laten vaccineren een positieve attitude ten aanzien van influenzavaccinatie en zij lieten ook sterkere gevoelens van autonomie in de vaccinatiebeslissing zien. Redenen voor acceptatie en weigering van influenzavaccinatie waren vergelijkbaar met uitkomsten van studies met gezondheidswerkers. De meest gerapporteerde redenen voor vaccinatie waren zelfbescherming, bescherming van patiënten en bescherming van familie en vrienden. De meest gerapporteerde redenen tegen vaccinatie waren een lage risicoperceptie (lage vatbaarheid en ernst) om influenza te krijgen, angst voor bijwerkingen, twijfel aan de effectiviteit van het vaccin, en dat vaccinatie nooit aangeboden werd. De resultaten suggereren het belang van het geven van onderwijs over influenza en vaccinatie vroeg in de geneeskunde opleiding en dat ziekenhuizen vaccins voor hun studenten beschikbaar moeten maken.

Hoofdstuk 4 beschrijft een kwalitatieve studie waarin 123 gezondheidswerkers uit België, Duitsland en Nederland geïnterviewd werden over de reden voor en tegen influenzavaccinatie, hun intentie om zich te laten vaccineren en mogelijke barrières voor vaccinatie. Het doel was om de determinanten en overtuigingen geassocieerd met de beslissing om zich tegen influenza te laten vaccineren te onderzoeken en een meer direct en dieper begrip van deze determinanten te krijgen dan wat door kwantitatieve studies vastgelegd kan worden. Daarnaast werden de overeenkomsten en verschillen van gezondheidswerkers uit drie naburige landen onderzocht. In alle landen werden zelfbescherming, bescherming van patiënten en bescherming van familieleden als de meest belangrijke redenen voor vaccinatie gerapporteerd. Redenen om zich niet te laten vaccineren waren angst voor bijwerkingen, een lage risicoperceptie, twijfels aan de effectiviteit van influenzavaccinatie, organisatorische barrières, een aantal misconcepties en ongedefinieerde negatieve gevoelens. We hebben drie overtuigingen geïdentificeerd die nauwelijks eerder beschreven zijn voor dit onderwerp: alternatieve preventie overtuigingen, de omission bias, en naturalistische opvattingen. De determinanten en overtuigingen die in de studies gevonden werden moeten kwantitatief onderzocht worden om te identificeren wat het relatief en gecombineerd belang is in het verklaren van intentie om zich als Gezondheidswerker te laten vaccineren, maar ook om te identificeren wat het belang is van overeenkomsten en verschillen tussen de drie landen.

Hoofdstuk 5 beschrijft een online vragenlijststudie die onderzoekt wat de relatieve en gecombineerde sterkte is van voorspellers uit eerder onderzoek, theorie en onze kwalitatieve studie (hoofdstuk 4) in het verklaren van de motivatie om zich tegen influenza te laten vaccineren onder Belgische, Duitse en Nederlandse gezondheidswerkers. Daarnaast geeft deze studie inzicht in de noodzaak van land-specifieke interventies om de influenzavaccinatiegraad onder gezondheidswerkers te verhogen. De uit- 
komsten gaven aan dat er drie groepen gezondheidswerkers zijn die door verschillende determinanten beïnvloed worden: gezondheidswerkers die geen intentie hebben om zich te laten vaccineren, gezondheidswerkers die nog onzeker zijn over vaccinatie en gezondheidswerkers die een sterk positieve intentie hebben. Attitude en vaccinatiegedrag in het verleden verklaren een grootte hoeveelheid variantie in de intentie om zich tegen influenza te laten vaccineren. Daarnaast verhogen een perceptie van negatieve sociale normen, het hebben van een omission bias, lage morele normen, ouder zijn, en contact met patiënten hebben de waarschijnlijkheid voor het hebben van geen intentie om zich te laten vaccineren vergeleken met onzeker zijn over vaccinatie. Als gezondheidswerkers een hoge waargenomen vatbaarheid hadden om influenza te krijgen, lage naturalistische overtuigingen hadden en een lagere motivatie rapporteerden om zich alleen maar wegens zelfbescherming te laten vaccineren, hadden zij eerder een positieve intentie dan dat ze onzeker waren over vaccinatie. Daarnaast laat het onderzoek zien dat Belgische gezondheidswerkers over het algemeen het hoogst op bevorderende factoren van vaccinatie-intentie scoren terwijl Nederlandse gezondheidswerkers het hoogst op belemmerende factoren scoren. Daarom zouden landspecifieke interventies, en een focus op verschillende determinanten afhankelijk van de intentie om zich te laten vaccineren van gezondheidswerkers effectief kunnen zijn in het verhogen van de vaccinatiegraad.

Hoofdstuk 6 beschrijft een online vragenlijststudie die de determinanten van de intentie om zich tegen influenza te laten vaccineren (beschreven in hoofdstuk 5 ) in een Nederlandse steekproef van gezondheidswerkers onderzoekt. Daarna is met een follow-up survey getest of de intentie om zich te laten vaccineren en de determinanten daarvan goede voorspellers zijn van het daadwerkelijke gedrag van deze gezondheidswerkers. Het doel was om de determinanten te identificeren die het meest waarschijnlijk zullen helpen om de vaccinatiegraad onder gezondheidswerkers te verhogen. Gezondheidswerkers met een sterke positieve intentie om zich tegen influenza te laten vaccineren leken eerder een positieve attitude ten aanzien van influenzavaccinatie te hebben en hebben zich in het verleden vaker tegen influenza laten vaccineren. Gezondheidswerkers die niet de intentie hebben om zich te laten vaccineren leken vaker een negatieve attitude te hebben, hadden vaker een sterk gevoel van autonomie in de beslissing om zich te laten vaccineren, lieten een omission bias zien, hadden minder gevoelens voor persoonlijke verantwoordelijkheid om patiënten door vaccinatie te beschermen, hadden sterkere zelfbeschermingsmotieven en leken vaker in het verleden gevaccineerd te zijn. De opgenomen determinanten dragen aanzienlijk bij aan de verklaring van intentie van gezondheidswerkers om zich tegen influenza te laten vaccineren, en intentie was een sterke voorspeller van daadwerkelijk vaccinatiegedrag.

Hoofdstuk 7 beoordeelt een strategie om de influenzavaccinatiegraad onder Nederlandse werknemers van een tertiair expertisecentrum voor de diagnose en behandeling van patiënten met chronisch orgaan falen te verhogen. Het primaire doel van de studie was om te bestuderen of de vaccinatiegraad onder gezondheidswerkers ver- 
hoogd kan worden door een manipulatie van de default optie: de helft van de gezondheidswerkers kreeg een email met een afspraak voor vaccinatie met de mogelijkheid om de afspraak af te zeggen of te wijzigen (opt-out conditie), terwijl de andere helft een email kreeg waarin uitgelegd werd dat gratis vaccins beschikbaar zijn en dat ze een afspraak voor vaccinatie kunnen maken (opt-in conditie). De resultaten laten geen statistisch aantoonbaar effect van conditie op het tegen influenza gevaccineerd zijn zien, waarschijnlijk omdat de statistische power te klein was om een effect te vinden. Desondanks is het verschil van $11.5 \%$ in de vaccinatiegraad tussen de condities vergelijkbaar met een andere studie die deze aanpak voor het verhogen van influenzavaccinatie gebruikt heeft. Daarnaast leken gezondheidswerkers in de opt-out conditie vaker een afspraak voor influenzavaccinatie te hebben wat de waarschijnlijkheid van gevaccineerd worden vergroot. Het gebruiken van de opt-out default procedure zou een goed alternatief kunnen zijn voor de complexe vaccinatiecampagnes zoals die voorgesteld zijn in de afgelopen jaren. De ontwikkelde strategie is vrij makkelijk te implementeren, laag in kosten en zou alsnog effecten die vergelijkbaar zijn met die van complexe campagnes kunnen laten zien als het onder de juiste omstandigheden uitgevoerd is.

Hoofdstuk 8 vat de resultaten van de studies samen en beschrijft de ontwikkeling en implementatie van het voorgestelde gedragsveranderingsprogramma om de influenza-vaccinatiegraad onder gezondheidswerkers te verhogen. Het geeft een overzicht van de sterktes en beperkingen van het onderzoeksproject en bespreekt praktische toepassingen en aanbevelingen voor toekomstig onderzoek. In het bijzonder is aanbevolen om de opt-out default strategie in ziekenhuizen te implementeren en om de impact daarvan over een langere tijdperiode te monitoren. Om effectief te kunnen zijn, moeten veranderingen in de procedure aan gezondheidswerkers gecommuniceerd worden, en zou het bewustzijn voor het belang van influenzavaccinatie een herhaalde educatieve inspanning moeten zijn. Daarnaast zouden gedragsveranderingsprogramma's ontwikkeld kunnen worden die op de doelgroepen gefocust zijn die gezondheidswerkers omringen en indirect beïnvloeden, zoals patiënten, ziekenhuisdirecties en de overheid. Maar het wordt ook gesuggereerd om verplichte vaccinatieprogramma's te overwegen omdat vrijwillige programma's niet het beoogde effect hebben op het vaccinatiegedrag. Tot slot is aanbevolen om de (sociale) media berichtgeving met betrekking tot influenzavaccinatie te monitoren, het gebruik van websites door zorgaanbieders te vergemakkelijken, en communicatiestrategieën te ontwikkelen die de negatieve media aandacht die influenzavaccinatie krijgt tegengaan. 


\section{Zusammenfassung}





\section{Zusammenfassung}

Jährliche Influenzaepidemien sind ein beträchtliches Problem für das Gesundheitswesen und verursachen eine hohe Morbidität und Mortalität. Betroffen sind vor allem folgende Risikogruppen: junge Kinder, ältere Menschen und Menschen mit chronischen Erkrankungen. Medizinischem Personal wird empfohlen, sich jährlich gegen Influenza impfen zu lassen, da dies eine Übertragung, besonders auf den gefährdeten Patientenkreis, reduziert und die Kosten im Gesundheitswesen senkt. Trotz des Effektivitätsnachweises der Impfung, der Verminderung von Krankheitsausfällen und der Reduzierung von Kosten sind die Impfraten unter medizinischem Personal niedrig. Ziel dieses Forschungsprojektes war es, bestehendes Wissen bezüglich der Bereitschaft von Krankenhauspersonal zur Influenzaimpfung zu erweitern und ein auf Theorie- und evidenzbasiertes Programm für Verhaltensänderung zu entwickeln, das die Impfquote in dieser Zielgruppe fördert. Die in dieser Arbeit vorgestellten Studien umfassen die Art und Weise der Kommunikation zu diesem Thema im Internet, die bestimmenden Faktoren für Medizinstudenten und Krankenhauspersonal in Bezug auf ihre Impfbereitschaft und ihr tatsächliches Impfverhalten sowie das zu diesem Thema erstellte Programm zur Verhaltensänderung, das in einem Pflegezentrum implementiert wurde.

Kapitel 2 beschreibt eine Studie, in der die Internetinhalte zum Thema Influenza und Influenzaimpfung auf niederländischen Nachrichten-Websites sowie in den sozialen Medien untersucht wurden. Das Ziel dieser Studie war es, Einblicke in die Kommunikation der Öffentlichkeit bezüglich Influenza zu gewinnen. In dem dreimonatigen Beobachtungszeitraum, in dem eine Influenzaepidemie stattfand, waren unterschiedliche Handhabungen zu erkennen. Drei übergreifende Themen wurden in den Informationen beider Quellen gefunden: (1) die Influenzaepidemie, (2) allgemeine Informationen zum Virus, Prävention und Behandlung und (3) Unsicherheit und Misstrauen gegenüber Influenzaimpfung. Diese Themenbereiche wurden in beiden Quellen ähnlich behandelt, jedoch unterschied sich die Kommunikation im Tonfall. Nachrichtenseiten wiesen eine neutralere und weniger wertende Berichterstattung auf, während soziale Medien die Schwere von Influenza und die Notwendigkeit zur Impfung eher skeptisch bewerteten. Medien könnten die Haltung der Öffentlichkeit gegenüber der Influenzaimpfung und damit den Erfolg von Impfkampagnen und Empfehlungen von Gesundheitsbehörden beeinflussen.

Kapitel 3 zeigt eine Fragenbogenstudie unter vorklinischen Medizinstudenten eines deutschen Universitätsklinikums. Das Ziel dieser Studie war es, mehr über die Faktoren für diese Gruppe herauszufinden, die die Absicht zur Influenzaimpfung vorhersagen sowie die Gründe für Akzeptanz und Ablehnung aufzeigen. Die Ergebnisse lassen erkennen, dass, verglichen mit noch unentschlossenen Medizinstudenten, die ohne Impfabsicht nicht dachten dass wichtige Andere (z.B. Familie, Kollegen) von ihnen erwarten sich impfen zu lassen und ließen ein stärkeres Gefühl von Autonomie in der Impfentscheidung erkennen. Verglichen mit denen, die noch unentschlossen sind, 
zeigten Medizinstudenten mit einer positiven Impfabsicht eine positive Haltung gegenüber der Influenzaimpfung und auch starke Gefühle von Autonomie in der Entscheidung für eine Impfung. Gründe für Akzeptanz und Ablehnung der Influenzaimpfung waren vergleichbar mit Kenntnissen aus anderen Studien mit medizinischem Personal. Die am häufigsten genannten Gründe für eine Impfung waren Selbstschutz, Schutz von Patienten sowie von Familie und Freunden, die am häufigsten genannten Gründe für eine Ablehnung eine niedrige Risikowahrnehmung (niedrige Anfälligkeit und Ernsthaftigkeit), Angst vor Nebenwirkungen, Zweifel an der Effektivität des Impfstoffes und das Fehlen eines Impfangebots. Diese Ergebnisse suggerieren, dass Aufklärung in Bezug auf Influenza und die Wichtigkeit der Impfung früh in der medizinischen Ausbildung stattfinden sollte und dass Krankenhäuser auch ihren Medizinstudenten Impfungen zur Verfügung stellen sollten.

Kapitel 4 enthält eine qualitative Studie, in der eine Gruppe von 123 belgischen, niederländischen und deutschen Krankenhausmitarbeitern über die Gründe für und gegen die Influenzaimpfung befragt wurde. Das Ziel war es zu untersuchen, welche bestimmenden Faktoren und Überzeugungen mit der Impfentscheidung zusammenhängen und eine direktere und gründlichere Einsicht zu gewinnen, als sie eine quantitative Studie erfassen kann. Des Weiteren wurden die Übereinstimmungen und Unterschiede zwischen dem Verhalten des medizinischen Personals aus drei benachbarten Ländern untersucht. In allen drei Ländern wurden der Selbstschutz und der Schutz von Patienten und Familienmitgliedern als wichtigste Gründe für die Impfung genannt, dagegen sprachen Angst vor Nebenwirkungen, eine niedrige Risikowahrnehmung, Zweifel an der Effektivität der Impfung, organisatorische Hürden, verschiedene Missverständnisse und undefinierbare, negative Gefühle. Drei Überzeugungen wurden identifiziert, die bisher zu diesem Thema weitgehend unbeschrieben sind: alternative Überzeugungen zum Schutz vor Infektionen, der sogenannte Unterlassungseffekt (omission bias) und naturalistische Auffassungen. Die in dieser Studie gefundenen bestimmenden Faktoren und Überzeugungen sollten noch quantitativ untersucht werden, um die relative und gemeinsame Wichtigkeit im Erklären der Impfabsicht von medizinischem Personal feststellen zu können sowie die Bedeutung der Gemeinsamkeiten und Unterschiede zwischen den drei Ländern.

Kapitel 5 beschreibt eine Online-Umfrage, die die relative und gemeinsame Stärke von Prädiktoren aus ehemaligen Studien, der Theorie und der in Kapitel 4 beschriebenen qualitativen Studie bezüglich der Impfabsicht von belgischen, niederländischen und deutschen Krankenhausmitarbeitern untersucht. Des Weiteren bietet diese Studie Einblick in die Notwendigkeit von landesspezifischen Interventionsprogrammen zur Steigerung der Influenzaimpfquote für medizinisches Personal. Die Ergebnisse zeigen auf, dass es drei durch verschiedene Faktoren beeinflusste Gruppen gibt: medizinisches Personal ohne Impfabsicht, unentschlossenes medizinisches Personal und medizinisches Personal mit einer stark positiven Impfabsicht. Die Haltung und das Impfverhalten in der Vergangenheit erklärten einen wesentlichen Teil der Varianz in der Ab- 
sicht, sich gegen Influenza impfen zu lassen. Darüber hinaus steigerten wahrgenommene negative soziale Normen, das Aufzeigen eines Unterlassungseffektes, niedrigere moralische Normen, höheres Lebensalter sowie fehlender Patientenkontakt die Wahrscheinlichkeit, sich gegen die Impfung zu entscheiden. Medizinisches Personal zeigte eine größere Wahrscheinlichkeit einer positiven Impfabsicht bei einer hohen wahrgenommenen Anfälligkeit für Influenza, niedrigen naturalistischen Ansichten und wenn Selbstschutz nicht als Hauptmotiv angesehen wurde. Die Ergebnisse zeigen außerdem, dass belgisches medizinisches Personal unterstützende Faktoren der Impfabsicht am höchsten bewertete, während niederländisches Personal vorwiegend hemmende Faktoren hoch bewertete. Daraus kann geschlossen werden, dass landesspezifische Interventionsprogramme ein größeres Potential zur Hebung der Impfbereitschaft aufweisen. Außerdem sollte berücksichtigt werden, dass abhängig von der Impfabsicht verschiedene Faktoren ausschlaggebend sind: Interventionsprogramme sollten verschiedene Gruppen ansprechen und individueller sein als bestehende Kampagnen.

Kapitel 6 beschreibt eine Online-Umfrage, in der die bestimmenden Faktoren der Impfabsicht (beschrieben in Kapitel 5) von niederländischem medizinischem Personal in einer Stichprobe gemessen wurden. Im Anschluss wurde mit einer Folge-Umfrage getestet, ob die Impfabsicht sowie die bestimmenden Faktoren gute Prädiktoren des tatsächlichen Impfverhaltens derselben Personengruppe sind. Das Ziel war es, die bestimmenden Faktoren zu identifizieren, die mit der größten Wahrscheinlichkeit die Impfquote fördern. Medizinisches Personal mit einer stark positiven Impfabsicht hatte mit höherer Wahrscheinlichkeit eine positive Haltung gegenüber der Influenzaimpfung und tendierte dazu, bereits in der Vergangenheit häufiger gegen Influenza geimpft worden zu sein. Medizinisches Personal ohne Impfabsicht zeigte mit höherer Wahrscheinlichkeit eine negative Haltung, starke Gefühle von Autonomie in der Impfentscheidung, einen Unterlassungseffekt, geringeres Empfinden persönlicher Verantwortung gegenüber Patienten sowie höhere Motivation zum Selbstschutz und tendierte in der Vergangenheit dazu, sich nicht impfen zu lassen. Die einbezogenen bestimmenden Faktoren trugen erheblich zur Erklärung der Impfabsicht von medizinischem Personal bei. Impfabsicht war wiederum ein starker Prädiktor des tatsächlichen Impfverhaltens.

Kapitel 7 beurteilt eine Strategie zur Förderung der Impfquote bei niederländischem Personal eines Pflegezentrums für die Diagnose und Behandlung von Patienten mit chronischem Organversagen. Das Hauptziel dieser Studie war es herauszufinden, ob die Impfquote bei medizinischem Personal durch Manipulation der StandardBedingung (default option) gefördert werden kann. Eine Hälfte des medizinischen Personals erhielt eine E-Mail mit einem festen Impftermin und der Möglichkeit, diesen über einen Link abzusagen oder zu verschieben (opt-out-Bedingung), während die andere Hälfte via E-Mail über eine kostenlose Impfung informiert wurde, aber einen Impftermin eigeninitiativ ausmachen musste (opt-in-Bedingung). Die Ergebnisse zeigen keinen statistisch feststellbaren Effekt der Bedingung auf das Influenzaimpfverhalten, möglicherweise durch die unzureichende statistische Power, die keinen Effekt finden 
ließ. Dennoch ist der Unterschied von $11.5 \%$ in der Impfquote zwischen den Versuchsbedingungen vergleichbar mit einer anderen Studie, die diesen Ansatz zur Förderung der Influenzaimpfung verwendet hat. Des Weiteren wählte das medizinische Personal in der opt-out-Bedingung mit einer höheren Wahrscheinlichkeit einen Termin zur Influenzaimpfung, was wiederum die Wahrscheinlichkeit, sich impfen zu lassen, erhöhte. Es wird darauf hingewiesen, dass das opt-out-default-Verfahren eine gute Alternative zu den in den letzten Jahren vorgeschlagenen komplexen Impfkampagnen sein könnte, da es relativ einfach umzusetzen und kostengünstig ist und Ergebnisse erzielen könnte, die mit komplexeren Kampagnen vergleichbar sind.

Kapitel 8 fasst die Resultate der Studien zusammen und beschreibt die Entwicklung und Umsetzung des vorgeschlagenen Programmes zur Verhaltensänderung. Es bietet eine Übersicht über die Stärken und Schwächen des Forschungsprojektes und bespricht praktische Auswirkungen und Empfehlungen für die zukünftige Forschung. Insbesondere wird empfohlen, die opt-out-default-Strategie in Krankenhäusern umzusetzen und deren Auswirkungen über einen längeren Zeitraum zu beobachten. Für eine erfolgreiche Umsetzung müssen Verfahrensänderungen offen unter medizinischem Personal besprochen werden. Das Schärfen des Bewusstseins für die Wichtigkeit der Influenzaimpfung sollte eine wiederkehrende didaktische Bemühung sein. Des Weiteren wird vorgeschlagen, Programme zur Verhaltensänderung zu entwickeln, die auf Zielgruppen wie Patienten, das Krankenhausmanagement und die Politik ausgerichtet sind, die das medizinische Personal indirekt beeinflussen. Allerdings wird auch empfohlen, verpflichtende Impfprogramme in Betracht zu ziehen, da freiwillige Programme nicht den gewünschten Effekt auf Impfquoten erzielen. Abschließend wird angeraten, die Berichterstattung bezüglich Influenzaimpfung in den Medien zu beobachten, die Nutzung von Websites des öffentlichen Gesundheitswesens zu verbessern und Kommunikationsstrategien zu entwickeln, die der negativen Aufmerksamkeit gegenüber der Influenzaimpfung in den Medien entgegenwirken. 
Acknowledgements 



\section{Acknowledgements}

Last, but not least, I want to acknowledge the people that were by my side during the process of writing this dissertation - without you it would not have been possible to finish this book, or at least it would have made the process so much harder.

Thank you, bedankt, Danke!

Mijn begeleiders Rob en Gerjo. Rob, bedankt voor de goede begeleiding. Ik heb ontzettend veel van je geleerd. Ook in moeilijke tijden heb je je rust bewaard en wist je me het gevoel te geven dat ik mijn best had gedaan. Gerjo, ik waardeer de manier waarop je geen blad voor de mond neemt en ik heb veel gehad aan je doortastende manier om voor- maar vooral tegenstanders te overtuigen van het belang van mijn onderwerp.

My supervisor Gretchen. Thank you so much for your kindness and hospitality. I felt very welcome when I came to visit you at Rutgers and I am very thankful for all the time you took for me. Without you and the countless brainstorms we had about my intervention, I cannot imagine what it would have turned out to be. I also would like to thank the MDM lab, Jeff and Haewon, for introducing me to all the other PhD-students and for making sure that I would have a good time in New Brunswick.

Meine Familie. Mama und Papa, Danke für das Ermöglichen von allen Erfahrungen die ich bis jetzt machen durfte. Eure bedingungslose Unterstützung und Euer ungebremstes Interesse an meiner Doktorarbeit haben mir sehr viel bedeutet und mir sehr geholfen.

Meine Schwester Anna. Du bist ein Vorbild für mich und ich bin dankbar, dass du immer da bist, wenn etwas Wichtiges in meinem Leben passiert.

Und meinen Großeltern für ihren Stolz und ihre Unterstützung.

Mijn collega's in Maastricht. Inge, Katharina, Annika, Suzanne, Tobias, Loes, Karlijn, Fraukje, Alicia, Philippe, Mariëlla, Irene, Saar, Anne, bedankt voor een leuke werksfeer, gekke uitjes, veel vlaai, dinner clubs en de "feministische Stammtisch".

In het bijzonder mijn kamergenootjes Sanne en Dilana. Bedankt voor de gezelligheid en goede gesprekken. Wij waren er ontzettend goed in thee, pepernootjes, kamer versiering, tegenslagen, successen en planten opvoeding te delen.

En Stefan voor de gezelligheid, bij voorkeur met goed eten, goede muziek, bier en een sigaretje. 
Arjan, bedankt voor de goede begeleiding bij de masterscriptie. Zonder jou was ik nooit de wetenschap ingegaan en zonder jouw aanmoedigingen was ik nooit aan een promotietraject begonnen.

Meine Kölner Mädels Britta, Nina und Scheku. Danke für eure Freundschaft und dass ihr mir immer das Gefühl gebt noch einen Platz in Köln zu haben. Sich auch nach Wochen des Nicht-Sehens so zu fühlen als wäre man nie weggewesen, ist etwas sehr wertvolles.

Meine Maastrichter Mädels Kristy, Suse, Sally, Anna, Annabell, Amrei und Medi - inzwischen verstreut in Deutschland und Holland - Danke für eure Freundschaft und das Erträglichmachen des Studiums.

Besonderen Dank an Kristy. Du hast alle Rückschläge und Erfolge hautnah mitbekommen. Du warst eine sehr wichtige und große Unterstützung. Unsere regelmäßigen gemeinsamen Essen, Gespräche, Kinobesuche und Nächte im FM Cafe werden mir sehr fehlen.

Thanks to everyone else who I did not mention, but who contributed, in whatever manner, to this work. 


\section{Curriculum Vitae}

Birthe Lehmann was born in Gelsenkirchen-Buer, Germany on the $26^{\text {th }}$ of August 1986. After completing her secondary school education in Cologne, she started studying Psychology at Maastricht University, in the Netherlands in 2007. During her Bachelor, she studied a semester abroad at Stellenbosch University in South Africa. In 2010, she finished her Bachelor and started the Health and Social Psychology Master at Maastricht University. During the Master, she spent a semester in Cologne, Germany for an internship and the completion of her thesis. In 2011, she obtained her Master's degree (summa cum laude) and started working as a junior researcher at the Work and Social Psychology Department, Maastricht University. In March 2012, she started her PhDproject, of which the results are described in this dissertation, at the same department. She worked on her PhD-project under supervision of Prof. Dr. Robert Ruiter, Prof. Dr. Gerjo Kok, and Prof. Dr. Gretchen Chapman, who she visited for four months in the Medical Decision Making Lab at Rutgers University, New Jersey, U.S. In July 2015, she started working as a researcher at the National Institute for Public Health and the Environment (RIVM) in Bilthoven, the Netherlands. 
$\not+1+\lambda+1+\not+\lambda+1+\not+1+\not+1+1+1$ $+1+\not+1+1+\not+1+1+\not+1+1+1+\not+$ $1+1+\not+1+1+1+\not+1+1+1+1+1+\ell$ $+\not+1+\not+1+1+1+1+1+\not+1+\not+1+$ $1+\not+1+1+1+1+\not+1+1+1+\not+\not+1$ $+1+\not+1+1+1+\not+1+\not+1+1+1+\not+$ $\not+1+1+\lambda+1+1+1+1+1+\not+1+1+1$ $+1+\not+1+1+\not+1+1+\not+1+1+1+\not+$ $\not+1+1+1+\not+\lambda+1+\not+1+\not+1+1+1$ $+1+\not+1+1+\not+1+1+\not+1+1+1+\not+$ $\lambda+1+\not+1+1+1+\not+1+1+1+1+1+\ell$ 